\title{
STUDY ON FREE VIBRATION OF CURVED GIRDER BRIDGES*
}

\author{
By Sadao Komatsu** and Hiroshi Nakai***
}

\section{SYNOPSIS}

The studies on free vibration of a curved girder bridge are much complicated because the bending vibrations in orthogonal directions are generally coupled with the torsional one. The object of this paper is to present the fundamental equation for a freely vibrating curved girder bridge with an unsymmetrical cross-section and to obtain an approximate solution of the frequency equation. From these results, the practical formulae for determining the natural frequencies are proposed. Furthermore, the validity of this theory is verified by comparison with the results obtained from the experimental studies.

\section{INTRODUCTION}

Recently a great many curved girder bridges have been constructed in Tokyo and Osaka, so it is very important to confirm the security of such girder bridges from a dynamical point of view.

A few dynamical analysis concerning these kind of bridges have already been reported. For instance, the vibration of a curved bar has been studied by G. Schumpich ${ }^{1}$ in disregard of the warping rigidity. A. Hirai and Y. Fukazawa ${ }^{2}$ analized the vibration of a curved bar by taking its warping rigidity into consideration. H. Yonezawa ${ }^{3)}$ in his dynamical studies treated the curved girder bridge as a fan-shaped plate. The above studies mainly dealt with simply supported curved girder bridges and it seems that no analysis has been given for the continuous curved girder bridges so far.

Now, if a curved girder bridge having an unsymmetrical cross-section vibrates freely, both the bending vibrations in two mutually perpendicular directions and the torsional vibration occur simul-

* The abstract of this paper has been reported at 19 th Annual Conference for Japan Soc. of Civ. Eng., May 1964.

** Dr.Eng. Professor, Dept. of Civ. Eng., Fac. of Eng., Osaka University, 9-210 Higashinoda-Cho Miyakojima-Ku, Osaka, Japan.

*** Lecture, Dept. of Civ. Eng., Fac. of Eng., Osaka City University, 459 Sugimoto-Cho Sumiyoshi-Ku, Osaka, Japan. taneously, since the longitudinal axis has curvature and the shear center of its cross-section does not coincide with the center of gravity. Then, that phenomenon is nothing but the coupled vibrations of bending and torsion. For the straight beams with nonsymmetrical cross-section the same kind of phenomena had already been pointed out and analyzed by S.P. Timoshenko(), J.M. Gere and Y.K. $\mathrm{Lin}^{5}$.

With regard to the usual curved girder bridge the cross-sections of which are more or less different in shape and size from those of ordinary straight girder bridges, so the following properties should be given attention :

1) As the stress resultants in the outside girder are greater than those in the inside girder, the former should be designed to have larger size than the latter.

2) For taking centrifugal force due to driving vehicles suitable cant must be provided on the floor.

For these reasons, the cross sectional shapes of almost all curved girder bridges will be made unsymmetrically. Accordingly, whenever the vibration occurs in a curved girder bridge, both bending and torsional vibrations are usually coupled. For the curved bridge, the deflections of the longitudinal axes and the twist about the shear center are not able to be independent each other owing to the curvature of bridge axis. Such coupled vibrations will appear frequently even if the cross-section has a symmetrical axis. Because of this fact, the free vibration of curved girder bridges may be essentialy distinguished from that of straight girder bridges.

Now, the fundamental statical equations ${ }^{\text {() }}{ }^{10}$ ) for stress resultants and displacements are fairly derived by considering peculiar characteristic of the curved girder bridges. On the basis of these equations we have derived the fundamental equations of motion for the free vibration of the curved girder bridges. However, it is very difficult to find its exact solution, and that the rigorous solution may be too complex to be applied practically to numerical calculations. Then, a solution for frequency equation is given here by means of Galerkin's method, so the solution together with its approximate formulae 
may be applied for determining the natural frequencies of general curved girder bridges.

Finally, some experimental studies have been made on a several curved girder bridges constructed near Osaka. The theoretical values were seen to be coincided well with the measured values. Therefore, the formulae given in this paper are recognized to be useful for analyzing the dynamical characteristics of the curved girder bridges.

\section{THEORETICAL STUDY}

\section{System of Coordinates}

In the cross-section of a general curved gider bridge, let the shear center, the center of figure, the center of gravity, and the loading point be denoted by the symbols $S, O_{n}, G$ and $P$ respectively, and these points be laid on the circumferences of the circles with radius $R_{S}, R_{O}, R_{G}$, and $R_{P}$ respectively, away from the center of curvature $O-O$ as shown in Fig. 1.

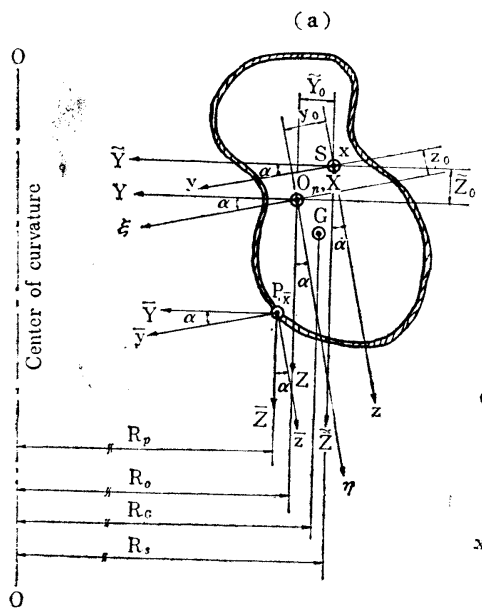

\section{(b)}

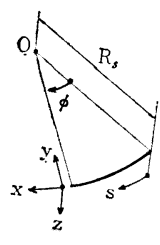

Fig. 1 Cross-section of curved girder bridge and system of coordinates.

In general, for the unsymmetrical cross-sections, the shear center $S$ does not coincide with the center of figure $O_{n}$, so the fundamental equations for determining stress resultants as well as displacements will become much complex. Accordingly, the following system of coordinates may be useful for the simplification of dealing with them.

First, let us take the right-hand rectangular coordinates $(X, Y, Z)$ through the center of figure $O_{n}$. The rectangular coordinates $X, Y$, and $Z$ are taken along the longitudinal axis through the center of figure of cross-section, in the radial inward, and in the vertical downward direction respectively. Second, the rectangular coordinates $(\xi, \eta)$ parallel to both principal axes through the same point $O_{n}$ are also specified. The principal axes $O_{n} \xi$ and $O_{n} \eta$ are inclined at an angle $\alpha$ to the axes $O_{n} Y$ and $O_{n} Z$ respectively. The magnitude of this angle $\alpha$ can easily be determined by the following formula ${ }^{8)}$,

$$
\alpha=\frac{1}{2} \tan ^{-1} \frac{2 I_{Y Z}}{I_{Z}-I_{Y}}
$$

where $I_{Y}, I_{Z}$, and $I_{Y Z}$ are the moments and product of inertia with respect to the axes $O_{n} Y, O_{n} Z$. Then the geometrical principal moments of inertia $I_{\xi}$ and $I_{\eta}$ can be estimated by ${ }^{8)}$

$$
I_{\xi \circ \mathrm{r} \eta}=\frac{1}{2}\left\{I_{Y}+I_{Z} \pm \sqrt{\left(I_{Y}-I_{Z}\right)^{2}+4 I_{Y Z^{2}}}\right\}
$$

Both orthogonal system of coordinates $(x, y, z)$ and $(x, \tilde{Y}, \tilde{Z})$ through the shear center $S$ are parallel to the axes $O_{n} X, O_{n} \xi, O_{n} \eta$ and $O_{n} X, O_{n} Y$, $O_{n} Z$ respectively, while the other pair of orthogonal ones $(\bar{x}, \bar{y}, \bar{z})$ and $(\bar{x}, \bar{Y}, \bar{Z})$ through the point $P$ are also parallel to the axes $S x, S y, S z$ and $S X$, $S \tilde{Z}, S \tilde{Z}$ respectively. Between the coordinates system $(y, z)$ and $(\tilde{Y}, \tilde{Z})$, the following relationship can be written

$$
\left.\begin{array}{l}
y=\tilde{Y} \cos \alpha+\tilde{Z} \sin \alpha, \\
z=-\tilde{Y} \sin \alpha+\tilde{Z} \cos \alpha .
\end{array}\right\}
$$

The coordinates $(\bar{y}, \bar{z})$ have similar relationship to $(\bar{Y}, \bar{Z})$.

Besides, the curvilinear coordinates $s$ are taken to specify the situation of the cross-section. If the symbol $\phi$ denotes the central angle made by any cross-section and the right end section of a curved girder bridge, the circumferential coordinates $s$ can be given by the following equation (Fig. 1(b)).

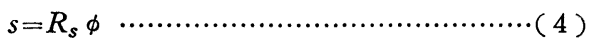

The necessary fundamental equations will be derived by using this curvilinear coordinates $s$.

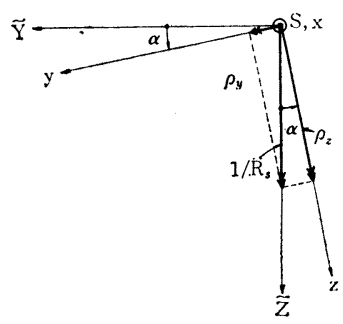

Fig. 2 Components of curvature.

Fig. 2 illustrates the components of curvature about the axes $S y$ and $S z$, so that

$$
\left.\begin{array}{l}
\rho_{y}=\sin \alpha / R_{s} \\
\rho_{z}=\cos \alpha / R_{s}
\end{array}\right\}
$$

2. Fundamental Differential Equation for Stress Resultants and Displacements ${ }^{6 / 7)}$

(1) Fundamental differential equation for stress resultants.

Fig. 3 shows internal forces at any cross-section $s$. 


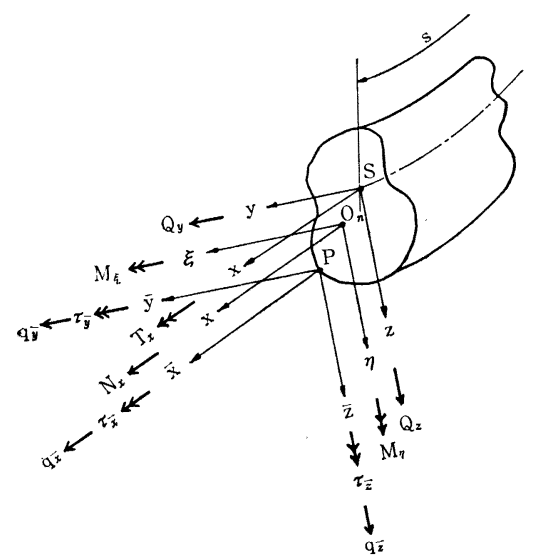

Fig. 3 Stress resultants.

There axial force along the axis $O_{n} X$ is denoted by $N_{X}$, two shearing forces along the axes $S y, S z$ by $Q_{y}, Q_{z}$, torsional moment about the axis $S X$ by $T_{x}$ and two bending moments about the axes $O_{n} \xi, O_{n} \eta$ by $M_{\xi}, M_{\eta}$ respectively.

In addition, the distributed external forces along the axes $P \bar{x}, P \bar{y}, P \bar{z}$ are represented by $q_{\bar{x}}, q_{\bar{y}}, q_{\bar{z}}$ and the torques about the axes $P \bar{x}, P \bar{y}, P \bar{z}$ by $\tau_{\bar{x}}$, $\tau_{\bar{y}}, \tau_{\bar{z}}$ respectively. The magnitudes of these resultants have positive values when they act in such directions as shown in Fig. 3.

Let us now consider the equilibrium of those stress resultants acting on the differential element $d s$, cut off by two ajacent sections $s$ and $s+d s$, as follows :

a) Equilibrium of the forces acting in the directions of axes $x, y$ and $z$.

By translating all the forces to the shear center axis the following linearized equations can be obtained :

$$
\left.\begin{array}{l}
\frac{d N_{X}}{d s}-\rho_{z} Q_{y}+\rho_{y} Q_{z}+\frac{R_{P}}{R_{S}} q_{\bar{x}}=0 \\
\frac{d Q_{y}}{d s}+\rho_{z} N_{X}+\frac{R_{P}}{R_{S}} q_{\bar{y}}=0 \\
\frac{d Q_{z}}{d s}-\rho_{y} N_{X}+\frac{R_{P}}{R_{S}} q_{\bar{z}}=0
\end{array}\right\}
$$

b) Equilibrium of the moments about the axes $x$, $y$ and $z$.

To find the equilibrium of the moments about the shear center axis, we must take into consideration not only external torques together with the stress.

Resultants, but also moments caused by the eccentricity of these forces. The results can be written as follows :

$$
\left.\begin{array}{r}
\frac{d T_{x}}{d s}-\rho_{z} M_{\xi}+\rho_{y} M_{\eta}-\left(\rho_{y} y_{o}+\rho_{z} z_{o}\right) N_{X} \\
+\frac{R_{P}}{R_{S}}\left(\tau_{\bar{x}}-z_{P} q_{\bar{y}}+y_{P} q_{\bar{z}}\right)=0
\end{array}\right)
$$

$$
\left.\begin{array}{l}
\frac{d M_{\xi}}{d s}+z_{o} \frac{d N_{X}}{d s}+\rho_{z} T_{x}-Q_{z}+\frac{R_{P}}{R_{S}} \tau_{\bar{y}}=0 \\
\frac{d M_{\eta}}{d s}-y_{o} \frac{d N_{X}}{d s}-\rho_{y} T_{x}+Q_{y}+\frac{R_{P}}{R_{S}} \tau_{\bar{z}}=0
\end{array}\right\}
$$

where the symbols $\left(y_{o}, z_{o}\right)$ and $\left(y_{P}, z_{P}\right)$ designate the coordinates of situations of the center of figure $O_{n}$, and of the loading point $P$ with respect to the system of coordinates $(y, z)$ respectively.

These coordinates can readily be found by Eq. (3), provided the other coordinates $\left(\tilde{Y}_{O}, \tilde{Z}_{O}\right)$ and $\left(\tilde{Y}_{P}\right.$, $\tilde{Z}_{P}$ ) are known.

If a curved girder bridge undergoes an arbitrary external force, we can readily solve the simultaneous equation (6) 1 3 $_{3}$ for the stress resultants $N_{X}, Q_{y}$ and $Q_{z}$. Then, by substituting these quantities into the simultaneous equations $(7)_{1 \sim 3}$, the stress resultants $T_{x}, M_{\xi}$ and $M_{\eta}$ will be able to be given.

However, for the case of the actual loading conditions of a curved girder bridge, any external loads except seismic force rarely act along the axial direction, so we can put for dead and traffic loads.

$$
q_{\bar{x}}=0
$$

If a force $q$ acts transversely and that is inclined at an angle $\delta$ from the vertical axis, its horizontal and vertical components $q \widetilde{Y}, q \widetilde{Z}$ in the directions of axes $O_{n} \tilde{Y}$ and $O_{n} \tilde{Z}$ can be found by the following formulae as shown in Fig. 4.

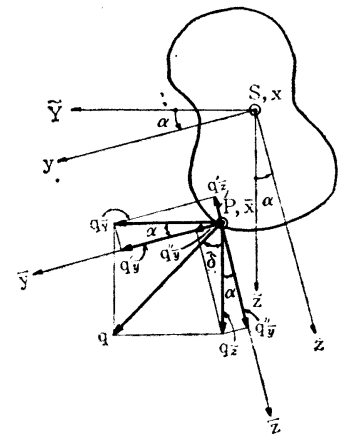

Fig. 4 Analysis of arbitrary force $q$.

$$
\left.\begin{array}{l}
q \widetilde{\boldsymbol{Y}}=q \sin \delta \\
q \widetilde{\boldsymbol{Z}}=q \cos \delta
\end{array}\right\}
$$

Then, $q \widetilde{Y}$ is again resolved in the directions of axes $\bar{y}, \bar{z}$,

$$
\begin{aligned}
& q_{\bar{y}^{\prime}}=q \cos \alpha \sin \delta \\
& q_{\bar{z}^{\prime}}=-q \sin \alpha \sin \delta
\end{aligned}
$$

and by substituting these into Eq. (6) ${ }_{1 \sim 3}$,

$$
\frac{d^{2} N_{X}}{d s^{2}}+\frac{N_{X}}{R_{S}^{2}}=-\frac{R_{P}}{R_{S}^{2}} q \sin \delta
$$

Next, the components $q_{\bar{y}^{\prime \prime}}$ and $q_{\bar{z}}{ }^{\prime \prime}$ of the force $q \widetilde{Z}$ will be given by

$$
\left.\begin{array}{l}
q_{\bar{y}^{\prime \prime}}=q \sin \alpha \cos \delta \\
q_{\bar{z}}^{\prime \prime}=q \cos \alpha \cos \delta
\end{array}\right\}
$$


In the same way, substituting Eq. (12) into Eq. (6) 1 3

$$
\frac{d^{2} N_{X}}{d s^{2}}+\frac{N_{X}}{R_{S^{2}}^{2}}=0
$$

The differential equation for the axial force can be decided by the above equation (13) when the force is vertically applied as a dead load. For the live load, the centrifugal forces would be produced due to the vehicles running parallel to the longitudinal curved axis of the bridge, but its quantity is so small compared with vertical load that it may be neglected. Therefore, it may be considered that the live load acts vertically. So, $\delta$ may be put to be equal to zero for the general curved girder bridge.

$$
\delta=0
$$

Consequently, the differential equation for the axial force of a curved bridge will be reduced to Eq. (13). The solution can be put,

$$
N_{X}=C_{1} \sin \phi+C_{2} \cos \phi
$$

As to the boundary condition for $N_{X}$, there is no axial force caused at either end $\phi=0$ and $\phi=\emptyset$ for the case when one end is fixed while the other can move freely in the axial direction. Thus, from the two conditions $N_{X(\phi=0)}=N_{X(\phi=\Phi)}=0$,

$$
N_{X}=0
$$

Namely, there is no axial force acting at any crosssection.

We discussed only a case when vertical force acts upon a curved girder bridge, because the deflected angle $\delta$ of the external load is also small in other practical cases of traffic loading. Accordingly, by eliminating the axial force, the following differential equations for the stress resultants $T_{x}, M_{\xi}$, and $M_{\eta}$ can be approximately obtained from Eq. $(6)_{2 \sim 3}$ and $(7)_{1 \sim 3}$.

$$
\left.\begin{array}{l}
\frac{d^{2} M_{\xi}}{d s^{2}}+\rho_{z}^{2} M_{\xi}-\rho_{y} \rho_{z} M_{\eta}= \\
-\frac{R_{P}}{R_{S}}\left\{q_{\bar{z}}-\rho_{z}\left(\tau_{\bar{x}}-z_{P} q_{\bar{y}}+y_{P} q_{\bar{z}}\right)\right\} \\
\frac{d^{2} M_{\eta}}{d s^{2}}+\rho_{y}{ }^{2} M_{\eta}-\rho_{y} \rho_{z} M_{\xi}= \\
\frac{R_{P}}{R_{S}}\left\{q_{\bar{y}}-\rho_{y}\left(\tau_{\bar{x}}-z_{P} q_{\bar{y}}+y_{P} q_{\bar{z}}\right)\right\} \\
\frac{d T_{x}}{d s}-\rho_{z} M_{\xi}+\rho_{y} M_{\eta}= \\
-\frac{R_{P}}{R_{S}}\left(\tau_{\bar{x}}-z_{P} q_{\bar{y}}+y_{P} q_{\bar{z}}\right)
\end{array}\right\}(17)_{1 \sim 3}
$$

In general $\tau_{\bar{y}}$ and $\tau_{\bar{z}}$ are the distributed torques.

\section{(2) Fundamental Differential equation for displacements.}

The displacements of the cross-section of a curved girder bridge in the directions of axes $S x, S y$, $S z$ are denoted by the symbols $u, v, w$ respectively, and also the rotation about the axis $S x$ by the sym-

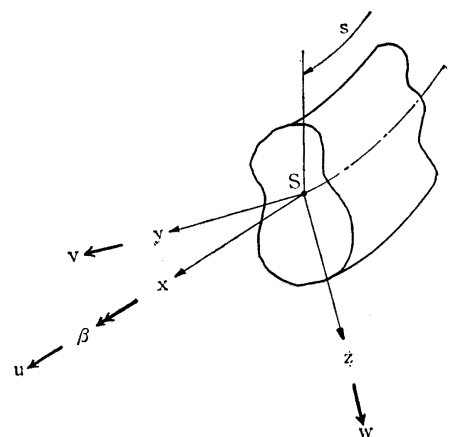

Fig. 5 Displacements $u, w$, $w$ and $\beta$.

bol $\beta$ as shown in Fig. 5. The sign of the displacements is governed by the same rule as in the forces.

These distortions refering to the shear center $S$ in the cross-section $s$ will produce the additional twisting angle $\vartheta$ and curvature $\rho_{y}{ }^{\prime}, \rho_{z}{ }^{\prime}$ about the shear center in the cross-section $s+d s$. If the radius of curvature of the unloaded girder is constant all over the span, these quantities may be written as follows;

$$
\left.\begin{array}{l}
\rho_{y}{ }^{\prime}=\rho_{y}+\rho_{z} \beta-\frac{d^{2} w}{d s^{2}}+\rho_{y} \frac{d u}{d s} \\
\rho_{z}{ }^{\prime}=\rho_{z}-\rho_{y} \beta+\frac{d^{2} v}{d s^{2}}+\rho_{z} \frac{d u}{d s} \\
\vartheta=\frac{d \beta}{d s}+\rho_{y} \frac{d v}{d s}+\rho_{z} \frac{d w}{d s}
\end{array}\right\}
$$

On the other hand, the axial strain $\varepsilon_{x}$ at the shear center has the following value.

$$
\varepsilon_{x}=\frac{d u}{d s}-\rho_{z} v+\rho_{y} w
$$

Now, by integrating Eq. (18) $)_{3}$ with respect to variable $s$ and omitting a constant term which means the rotation as the rigid body,

$$
\theta=\beta+\rho_{y} v+\rho_{z} w
$$

where the notation $\theta$ is called as the torsional angle.

Next, by the reference (8),

$$
\left.\begin{array}{l}
M_{\xi}=\frac{R_{S}}{R_{O}} E_{s} I_{\xi}\left(\rho_{y}{ }^{\prime}-\rho_{y}\right) \\
M_{\eta}=\frac{R_{S}}{R_{O}} E_{s} I_{\eta}\left(\rho_{z}{ }^{\prime}-\rho_{z}\right) \\
T_{x}=G_{s} J \frac{d \theta}{d s}-E_{s} C_{w} \frac{d^{3} \theta}{d s^{3}}
\end{array}\right\}
$$

where $J$ : torsion constant

$C_{w}$ : warping constant

$E_{s}$ : Young's modulus

$G_{s}$ : shear modulus of elasticity.

The first term on the right-hand side of Eq. $(21)_{3}$. is named St. Venant's torsional moment $T_{s}$ and second term is the secondary torsional moment $T_{w}$ due to warping, 


$$
\begin{aligned}
& T_{s}=G_{s} J \frac{d \theta}{d s} \\
& T_{w}=-E_{s} C_{w} \frac{d^{3} \theta}{d s^{3}}
\end{aligned}
$$

and the stress resultant $M_{w}$ is the warping moment,

$$
M_{w}=E_{s} C_{w} \frac{d^{2} \theta}{d s^{2}}
$$

The strain $\varepsilon_{x}$ along the axis $S x$ can also be ex. pressed by using $N_{X}, M_{\xi}, M_{\eta}$ and $M_{w}$ as follows ${ }^{8)}$

$$
\begin{aligned}
\varepsilon_{x}= & \frac{R_{O}}{R_{S}} \frac{1}{E_{s}}\left(\frac{N_{X}}{A_{s}}-\frac{M_{\xi}}{I_{\xi}} \eta_{s}-\frac{M_{\eta}}{I_{\eta}} \xi_{s}\right) \\
& +\frac{M_{w}}{E_{s} C_{w}} \cdot W_{s} \ldots \ldots \ldots \ldots \ldots \ldots \ldots \ldots \ldots \ldots \ldots \ldots \ldots \ldots \ldots \ldots \ldots \ldots
\end{aligned}
$$

where $A_{s}$ : cross sectional area

$\xi_{s}, \eta_{s}$ : distances between points $O_{n}$ and $S$ in the directions of axes $O_{n} \xi, O_{n} \eta$ respectively.

Since the value $W_{S}, i . e$. the warping at the shear center, must be equal to zero and the axial force of a usual curved girder bridge is small enough to be ignored as was mentioned before, the strain $\varepsilon_{x}$ can be evaluated from Eq. (21) and (24) as

$$
\varepsilon_{x}=-\left\{\left(\rho_{y}{ }^{\prime}-\rho_{y}\right) \eta_{S}+\left(\rho_{z}{ }^{\prime}-\rho_{z}\right) \xi_{S}\right\}
$$

Furthermore, by substituting Eq. (18) and (19) into the above equation, the relationship among displacements $u, v, w$ and $\beta$ will be found,

$$
\begin{aligned}
& \frac{1}{R_{S}} \frac{d u}{d s}=\frac{1}{1+\left(\frac{\eta_{S}}{R_{S}} \sin \alpha+\frac{\xi_{S}}{R_{S}} \cos \alpha\right)} \\
& \quad \times\left\{\frac{1}{R_{S}}\left(\rho_{z} v-\rho_{y} w\right)+\left(\frac{\xi_{S}}{R_{S}} \sin \alpha-\frac{\eta_{S}}{R_{S}} \cos \alpha\right)\right. \\
& \left.\quad \times \beta-\frac{\xi_{S}}{R_{S}} \cdot \frac{d^{2} v}{d s^{2}}+\frac{\eta_{S}}{R_{S}} \cdot \frac{d^{2} w}{d s^{2}}\right\} \cdots \cdots \cdots \cdots(25)
\end{aligned}
$$

Therefore, by making use of Eq. (25) in (18) and by substituting it into Eq. (21) 1 3 the relation between the displacements $v, w, \beta$ and the stress resultants
$M_{\xi}, M_{\eta}, T_{x}$ will be given.

In an ordinary curved girder bridge, however, the eccentric distances $\xi_{S}$ and $\eta_{S}$ are negligibly small as compared with the radius of curvature $R_{S}$. Then, by omitting the terms $\xi_{S} / R_{S}$ and $\eta_{S} / R_{S}$ in Eq. (25), the third term on the right-hand side of Eq. (18) can be described by using Eq. (5).

$$
\left.\begin{array}{l}
\rho_{y} \frac{d u}{d s} \cong \rho_{y} \rho_{z} v-\rho_{y}{ }^{2} w \\
\rho_{z} \frac{d u}{d s} \cong \rho_{z}^{2} v-\rho_{y} \rho_{z} w
\end{array}\right\}
$$

Applying these equations together with Eq. (18) $)_{1 \sim 3}$ as well as Eq. (21) 1 3, the linearlized formulae for the stress resultants $M_{\xi}, M_{\eta}$ and $T_{x}$ can be obtained as follows;

$$
\begin{aligned}
M_{\xi}= & -E_{s} I_{\xi}^{\prime}\left(\frac{d^{2} w}{d s^{2}}+\rho_{y}^{2} w-\rho_{y} \rho_{z} v-\rho_{z} \beta\right) \\
M_{\eta}= & E_{s} I_{\eta}{ }^{\prime}\left(\frac{d^{2} v}{d s^{2}}+\rho_{z}^{2} v-\rho_{y} \rho_{z} w-\rho_{y} \beta\right) \\
T_{x}= & G_{s} J\left(\frac{d \beta}{d s}+\rho_{y} \frac{d v}{d s}+\rho_{z} \frac{d w}{d s}\right) \\
& -E_{S} C_{w}\left(\frac{d^{3} \beta}{d s^{3}}+\rho_{y} \frac{d^{3} v}{d s^{3}}+\rho_{z} \frac{d^{3} w}{d s^{3}}\right)
\end{aligned}
$$

where new symbols $I_{\xi}^{\prime}$ and $I_{\eta}^{\prime}$ are

$$
\begin{aligned}
& I_{\xi}{ }^{\prime}=\frac{R_{S}}{R_{0}} I_{\xi} \\
& I_{\eta}{ }^{\prime}=\frac{R_{S}}{R_{0}} I_{\eta}
\end{aligned}
$$

\section{(3) Fundamental Differential Equation for Displacements and External Forces.}

By substituting Eq. (17) $)_{1 \sim 3}$ into Eq. (27) $)_{1 \sim 3}$ and eliminating the stress resultants, the linearlized basic differential equation for the displacements under the external forces can directly be written as follows;

$$
\begin{aligned}
& E_{s} I_{\eta}{ }^{\prime}\left[\frac{d^{4} v}{d s^{4}}+\frac{1}{R_{s}{ }^{2}} \cdot \frac{d^{2} v}{d s^{2}}+\left(\rho_{y} \rho_{z}\right)^{2} \cdot\left(1-\frac{I_{\xi}^{\prime}}{I_{\eta}^{\prime}}\right) v-\rho_{y} \rho_{z}\left(1-\frac{I_{\xi}^{\prime}}{I_{\eta}^{\prime}}\right)\left(\frac{d^{2} w}{d s^{2}}+\rho_{y}{ }^{2} w\right)\right. \\
& \left.-\rho_{y} \cdot\left\{\frac{d^{2} \beta}{d s^{2}}+\left(\rho_{y}{ }^{2}+\rho_{z}{ }^{2} \frac{I_{\xi}^{\prime}}{I_{\eta}^{\prime}}\right) \beta\right\}\right]=\frac{R_{P}}{R_{S}} \cdot\left\{q_{\bar{y}}-\rho_{y}\left(\tau_{\bar{z}}-z_{P} q_{\bar{y}}+y_{P} q_{\bar{z}}\right)\right\} \\
& E_{s} I_{\xi}^{\prime}\left[\frac{d^{4} w}{d s^{4}}+\frac{1}{R_{s}{ }^{2}} \cdot \frac{d^{2} w}{d s^{2}}+\left(\rho_{y} \rho_{z}\right)^{2} \cdot\left(1-\frac{I_{\eta^{\prime}}^{\prime}}{I_{\xi}^{\prime}}\right) w-\rho_{y} \rho_{z}\left(1-\frac{I_{\eta}^{\prime}}{I_{\xi}^{\prime}}\right)\left(\frac{d^{2} v}{d s^{2}}+\rho_{z}{ }^{2} v\right)\right. \\
& \left.-\rho_{z} \cdot\left\{\frac{d^{2} \beta}{d s^{2}}+\left(\rho_{z}{ }^{2}+\rho_{\nu}{ }^{2} \frac{I_{\eta}^{\prime}}{I_{\xi}^{\prime}}\right) \beta\right\}\right]=\frac{R_{P}}{R_{S}} \cdot\left\{q_{\bar{z}}-\rho_{z}\left(\tau_{\bar{x}}-z_{P} q_{\bar{y}}+y_{P} q_{\bar{z}}\right)\right\} \\
& E_{s} C_{w}\left[\frac{d^{4} \beta}{d s^{4}}-\frac{G_{s} J}{E_{s} C_{w}} \cdot \frac{d^{2} \beta}{d s^{2}}+\left(\rho_{z}^{2} \frac{I_{\xi}^{\prime}}{C_{w}}+\rho_{y}{ }^{2} \frac{I_{\eta}^{\prime}}{C_{w}}\right) \beta+\rho_{y}\left\{\frac{d^{4} v}{d s^{4}}-\left(\frac{G_{s} J}{E_{s} C_{w}}+\frac{I_{\eta}^{\prime}}{C_{w}}\right) \frac{d^{2} v}{d s^{2}}\right.\right. \\
& \left.\left.+\rho_{z}^{2}\left(\frac{I_{\xi}^{\prime}}{C_{w}}-\frac{I_{\eta}^{\prime}}{C_{w}}\right) v\right\}+\rho_{z}\left\{\frac{d^{4} w}{d s^{4}}-\left(\frac{G_{s} J}{E_{s} C_{w}}+\frac{I_{\xi}^{\prime}}{C_{w}}\right) \cdot \frac{d^{2} w}{d s^{2}}+\rho_{y}{ }^{2}\left(\frac{I_{\eta}^{\prime}}{C_{w}}-\frac{I_{\xi}^{\prime}}{C_{w}}\right) w\right\}\right] \\
& =\frac{R_{P}}{R_{S}}\left(\tau_{\bar{z}}-z_{P} \cdot q_{\bar{y}}+y_{P} q_{\bar{z}}\right) \text {. }
\end{aligned}
$$

\section{(4) Practical Formulae for Statical Prob- lem.}

For the case when a curved girder bridge undergoes an arbitrary external force at any situation, the simultaneous differential equations for stress resultants and displacements have been given previuosly, but their solutions might be much complicated.

However, if we consider again a statical problem in the case of only vertical load, both the shearing 


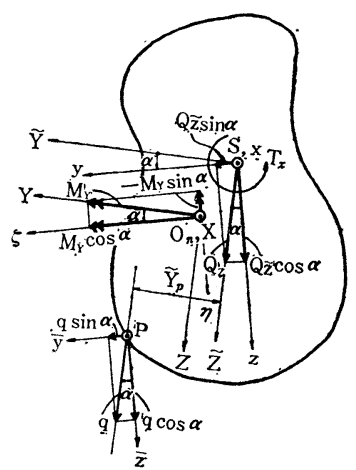

Fig. 6 Analysis of forces and stress resultants.

force and the bending moment can be given as shown in reference (11).

First, the components of a vertical force $q$ in the directions of axes $P \bar{y}$ and $P \bar{z}$ as shown in Fig. 6 are given by following formulae :

$$
\left.\begin{array}{l}
q_{\bar{y}}=q \sin \alpha \\
q_{\bar{z}}=q \cos \alpha
\end{array}\right\}
$$

The shearing force $Q \widetilde{Z}$ acting along the vertical axis $S \tilde{z}$ can be resolved into two shearing forces $Q_{y}$ and $Q_{z}$,

$$
\left.\begin{array}{l}
Q_{y}=Q_{\widetilde{Z}} \sin \alpha \\
Q_{z}=Q_{\widetilde{Z}} \cos \alpha
\end{array}\right\}
$$

On the other hand, the bending moment $M_{Y}$ about the horizontal axis $O_{n} Y$ can be resolved into two bending moments $M_{\xi}$ and $M_{\eta}$.

$$
\left.\begin{array}{l}
M_{\xi}=M_{Y} \cos \alpha \\
M_{\eta}=-M_{Y} \sin \alpha
\end{array}\right\}
$$

If the Eq. (30), (31) and (32) are substituted into the Eq. (6) $)_{1 \sim 3}$ and $(17)_{1 \sim 2}$ the equation for $Q \widetilde{Z}$ will be reduced to

$$
\frac{d Q_{\tilde{Z}}}{d s}=-\frac{R_{P}}{R_{S}} q
$$

while by using Eq. (3) the equation for $M_{Y}$ will be written as follows :

$$
\frac{d^{2} M_{Y}}{d s^{2}}+\frac{M_{Y}}{R_{S}^{2}}=-\left(\frac{R_{P}}{R_{S}}\right)^{2} \cdot q
$$

These equations show that the shearing force $Q_{\tilde{Z}}$ and bending moment $M_{Y}$ can be determined indepently.

Second, the differential equation for the torsional moment $T_{x}$ can be evaluated by applying Eq. (3), (30) and (32) to Eq. (17) $)_{3}$, and by using the solution $M_{Y}$ in Eq. (34)

$$
\frac{d T_{x}}{d s}=\frac{M_{Y}}{R_{S}}-\frac{R_{P}}{R_{S}} \tilde{Y}_{P} \cdot q
$$

where the symbol $\tilde{Y}_{P}$ designates the horizontal distance between points $S$ and $P$.

Then, by using Eq. (35), a differential equation for the warping moment can readily be derived. Thus, from both Eq. (21) ${ }_{3}$ and (23)

$$
\frac{d^{2} M_{w}}{d s^{2}}-\frac{G_{s} J}{E_{s} C_{w}} M_{w}=\frac{R_{0}}{R_{S}} \tilde{Y}_{P} q-\frac{M_{Y}}{R_{S}}
$$

is obtained. It is easily to find the solution $M_{w}$ under the specified boundary conditions.

The torsional angle $\theta$ can be given by

$$
\theta=\iint \frac{M_{w}}{E_{s} C_{w}}(d s)^{2}+C_{1} s+C_{2}
$$

Accordingly, the St. Venant's torsional moment $T_{s}$ and the secondary torsional moment $T_{w}$ can easily be found by means of Eq. (22) ${ }_{1 \sim 2}$, then the torsional moment $T_{x}$ is nothing but the sum of both the torsional moments.

$$
T_{x}=T_{s}+T_{w}
$$

The solutions for corresponding variation of these stress resultants to a number of typical loads had already been given ${ }^{11)}$ 13).

Next, the differential equation for the displacements $v$ and $w$ can be obtained by applying the solution of the bending moments $M_{\xi}, M_{\eta}$ and of the torsional angle $\theta$. Thus, from Eq. (20) and (27) $1 \sim 2$

$$
\begin{aligned}
& \frac{d^{2} v}{d s^{2}}+\frac{v}{R_{S^{2}}{ }^{2}}=\frac{M_{\eta}}{E_{s} I_{\eta}{ }^{\prime}}+\rho_{y} \theta \ldots \\
& \frac{d^{2} w}{d s^{2}}+\frac{w}{R_{S^{2}}{ }^{2}}=-\frac{M_{\xi}}{E_{s} I_{\xi}^{\prime}}+\rho_{z} \theta
\end{aligned}
$$

These equations indicate that both the displacements $v$ and $w$ will occur in the directions of principal axes in spite of the force applied only in the vertical direction. In general case, the displacements $v$, $w$ and $\theta$ are not so easily found as the stress resultants $Q_{\widetilde{Z}}$ or $M_{Y}$, because they are closely related to such cross sectional quantities as the bending rigidities $E_{s} I_{\xi}{ }^{\prime}, E_{s} I_{\eta}{ }^{\prime}$, the torsional rigidity $G_{s} J$ and the warping rigidity $E_{s} C_{w}$, as is seen in Eq. $(27)_{1 \sim 3}$ or (37), (39) and (40).

While the rotation angle $\beta$ of the cross-section can be obtained from Eq. (20) by making use of the solutions of $\theta, v$ and $w$.

$$
\beta=\theta-\left(\rho_{y} v+\rho_{z} w\right)
$$

\section{Fundamental Equation for Free Vibration of a Curved Girder Bridge.}

As to the problem of the free vibration of a curved girder bridge, let us start with the fundamental equations $(29)_{1 \sim 3}$ in which the axial displacement $u$ may be ignored as its effect is very small. Strictly speaking, the deformations due to the shearing forces and axial force should, of course, be taken in to account together. However, the effects upon the free vibration are so small as to be neglected. So far as we deal with the free vibration of a curved girder bridge, we may now principally consider it as the coupled vibrations of bending and twisting.

Accordingly, the fundamental differential equa- 
tions for a freely vibrating curved girder bridge can readily be derived on the basis of d'alambert's principal by replacing the external forces by the inertia forces.

Let us suppose the shear center $S$ is displaced by $v$ and $w$ in the directions of axes $y$ and $z$ respectively, and also the cross-section rotates about the axis $S x$ by angle $\beta$ as illustrated in Fig. 7. That is

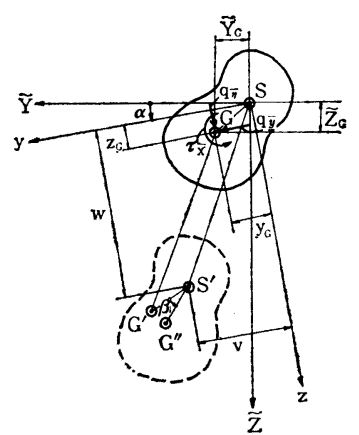

Fig. 7 Distortions of cross-section.

to say, the shear center $S$ will move to the point $S^{\prime}$ and the cross-section rotates by angle $\beta$ about the point $S^{\prime}$. At the same time, the center of gravity $G$ will change its position for the new point $G^{\prime \prime}$. If the coordinates of the center of gravity $G$ with reference to axes $S y, S z$ are denoted by $y_{G}, z_{G}$ respectively, the displacements $v_{G}, w_{G}$ of the point $G$ parallel to $S y, S z$ will be written as follows;

$$
\left.\begin{array}{l}
v_{G}=v-z_{G} \cdot \beta \\
w_{G}=w+y_{G} \cdot \beta
\end{array}\right\}
$$

On the other hand, the eccentricity $y_{G}$ and $z_{G}$ can also be given in the similar form as Eq. (3), provided the coordinates $\tilde{Y}_{G}, \tilde{Z}_{G}$ of the point $G$ with reference to $S \tilde{Y}, S \tilde{Z}$ are known.
To find the inertia forces $q_{\bar{y}}, q_{\bar{z}}$ and $\tau_{\bar{x}}$ at the center of gravity $G$, differentiating partially the displacements of Eq. (42) twice with respect to time $t$,

$$
\begin{aligned}
q_{\bar{y}} & =-\frac{A_{s} \gamma_{s}}{g} \cdot \frac{\partial^{2}}{\partial t^{2}}\left(v-z_{G} \beta\right) \\
q_{\bar{z}} & =-\frac{A_{s} \gamma_{s}}{g} \cdot \frac{\partial^{2}}{\partial t^{2}}\left(w+y_{G} \beta\right) \\
\tau_{\bar{x}} & =-\frac{I_{G} \gamma_{s}}{g} \cdot \frac{\partial^{2} \beta}{\partial t^{2}}
\end{aligned}
$$

where $r_{s}$ : density of steel material

$g$ : accelaration of gravity

$I_{G}$ : polar moment of inertia of the crosssection with respect to the center of gravity $G$.

For the sake of convenience, we put

$$
\begin{aligned}
& m=\frac{R_{G}}{R_{S}} \cdot \frac{r_{s}}{g} \ldots \ldots \ldots \ldots \\
& I_{S}=I_{G}+A_{s}\left(y_{G}{ }^{2}+z_{G}{ }^{2}\right)
\end{aligned}
$$

and substitute Eq. (43) into Eq. (29) 1 3 . Then, let us take into consideration the influence of curvature, and denote the projected cross sectional area to each axis by the symbol $A$ with the suffix $y, z$

$$
\begin{aligned}
& A_{y}=A_{s}\left(1+\rho_{y} \cdot z_{G}\right) \\
& A_{z}=A_{s}\left(1-\rho_{z} \cdot y_{G}\right) \\
& A_{y z}=A_{s} \cdot y_{G} \cdot \rho_{y} \\
& A_{y z}{ }^{\prime}=-A_{s} \cdot z_{G} \cdot \rho_{z}
\end{aligned}
$$

Likewise, let the symbol $S$ represents the geometrical moment of area,

$$
\left.\begin{array}{l}
S_{y}=A_{s} z_{G} \\
S_{y}{ }^{\prime}=A_{s} z_{G}+\rho_{y} I_{S} \\
S_{z}=A_{s} y_{G} \\
S_{z}{ }^{\prime}=A_{s} \cdot y_{G}-\rho_{z} \cdot I_{s}
\end{array}\right\}
$$

Consequently, when a curved girder bridge vibrates freely, the fundamental equation will be rewritten in the following form by using the preceding notations,

$$
\begin{aligned}
& \left.E_{s} I_{\eta}^{\prime}\left\{\frac{\partial^{4} v}{\partial s^{4}}+\frac{1}{R_{S^{2}}} \cdot \frac{\partial^{2} v}{\partial s^{2}}+\left(\rho_{y} \rho_{z}\right)^{2}\left(1-\frac{I_{\xi}^{\prime}}{I_{\eta}^{\prime}}\right) v\right\}+m A_{y} \frac{\partial^{2} v}{\partial t^{2}}-E_{s} I_{\eta}^{\prime} \rho_{y} \rho_{z}\left(1-\frac{I_{\xi}^{\prime}}{I_{\eta}^{\prime}}\right) \cdot\left(\frac{\partial^{2} w}{\partial s^{2}}+\rho_{y}^{2} w\right)\right] \\
& -m A_{y z} \frac{\partial^{2} w}{\partial t^{2}}-E_{s} I_{\eta}^{\prime} \rho_{y}\left\{\frac{\partial^{2} \beta}{\partial s^{2}}+\left(\rho_{y}{ }^{2}+\rho_{z}{ }^{2} \frac{I_{\xi}{ }^{\prime}}{I_{\eta}^{\prime}}\right) \beta\right\}-m S_{y}{ }^{\prime} \frac{\partial^{2} \beta}{\partial t^{2}}=0 \\
& E_{s} I_{\xi}^{\prime}\left\{\frac{\partial^{4} w}{\partial s^{4}}+\frac{1}{R_{S}^{2}} \cdot \frac{\partial^{2} w}{\partial s^{2}}+\left(\rho_{y} \rho_{z}\right)^{2}\left(1-\frac{I_{\eta}^{\prime}}{I_{\xi}^{\prime}}\right) w+m A_{z} \frac{\partial^{2} w}{\partial t^{2}}-E_{s} I_{\xi}^{\prime} \rho_{y} \rho_{z}\left(1-\frac{I_{\eta}^{\prime}}{I_{\xi}{ }^{\prime}}\right) \cdot\left(\frac{\partial^{2} v}{\partial s^{2}}+\rho_{z}{ }^{2} v\right)\right. \\
& -m A_{y z}{ }^{\prime} \frac{\partial^{2} v}{\partial t^{2}}-E_{s} I_{\xi}^{\prime} \rho_{z}\left\{\frac{\partial^{2} \beta}{\partial t^{2}}+\left(\rho_{z}{ }^{2}+\rho_{y}{ }^{2} \frac{I_{\eta}^{\prime}}{I_{\xi}^{\prime}}\right) \beta\right\}+m S_{z}{ }^{\prime} \frac{\partial^{2} \beta}{\partial t^{2}}=0 \\
& E_{s} C_{w}\left\{\frac{\partial^{4} \beta}{\partial s^{4}}-\frac{G_{S} J}{E_{S} C_{w}} \cdot \frac{\partial^{2} \beta}{\partial s^{2}}+\left(\rho_{z}^{2} \frac{I_{\xi}^{\prime}}{C_{w}}+\rho_{y}{ }^{2} \frac{I_{\eta}^{\prime}}{C_{w}}\right) \beta\right\}+m I_{S} \frac{\partial^{2} \beta}{\partial t^{2}} . \\
& +E_{s} C_{w} \rho_{y}\left\{\frac{\partial^{4} v}{\partial s^{4}}-\left(\frac{G_{s} J}{E_{s} C_{w}}+\frac{I_{\eta}{ }^{\prime}}{C_{w}}\right) \cdot \frac{\partial^{2} v}{\partial s^{2}}+\rho_{z}{ }^{2}\left(\frac{I_{\xi}^{\prime}}{C_{w}}-\frac{I_{\eta}^{\prime}}{C_{w}}\right) v\right\}-m S_{y} \frac{\partial^{2} v}{\partial t^{2}} \\
& +E_{s} C_{w} \rho_{z}\left\{\frac{\partial^{4} w}{\partial s^{4}}-\left(\frac{G_{s} J}{E_{s} C_{w}}+\frac{I_{\xi}^{\prime}}{C_{w}}\right) \cdot \frac{\partial^{2} w}{\partial s^{2}}+\rho_{y}^{2}\left(\frac{I_{\eta}^{\prime}}{C_{w}}-\frac{I_{\xi}^{\prime}}{C_{w}}\right) w\right\}+m S_{z} \frac{\partial^{2} w}{\partial t^{2}}=0
\end{aligned}
$$

These simultaneous differential equations are the basic ones for the free vibration of a usual curved girder, from which the frequency equation for any special case will be derived. By the way a specified problem can be simplified by virtue of the geometrical conditions for any usual girder bridge. For the present the solution of Eq.(48) ${ }_{1 \sim 3}$ corresponding to the most general coupled vibrations will be found 
in the following sections.

\section{Frequency Equation of usual Curved Gir- der Bridge.}

Generally, the displacements of a freely vibrating curved girder bridge may be represented with the product of the shape functions $\tilde{v}(s), \widetilde{w}(s), \widetilde{\beta}(s)$ by the time function $\mathfrak{q}(t)$ as follows;

$$
\left.\begin{array}{l}
v(s, t)=\tilde{v}(s) \cdot \mathrm{q}(t) \\
w(s, t)=\widetilde{w}(s) \cdot \mathrm{q}(t) \\
\beta(s, t)=\tilde{\beta}(s) \cdot \mathrm{q}(t)
\end{array}\right\}
$$

where the time function may be considered to be sinusoidal without damping

$$
\begin{aligned}
\mathrm{q}(t)= & \sin p_{i} t+\cos p_{i} t \\
& (i=1,2,3, \cdots \cdots)
\end{aligned}
$$

The symbol $p_{i}$ represents a circular frequency, so the relationship between the natural frequency $f_{i}$ and the period of free vibration $T_{i}$ can be written as follows;

$$
\begin{aligned}
& f_{i}=p_{i} / 2 \pi=1 / T_{i} \\
& T_{i}=2 \pi / p_{i}=1 / f_{i}
\end{aligned}
$$

Now, by substituting Eq. (48) $)_{1 \sim 3}$, the simultaneous ordinary differential equations for the shape functions $\tilde{v}, \widetilde{w}$ and $\tilde{\beta}$ are easily obtained as follows :

$$
\begin{aligned}
& E_{s} I_{\eta^{\prime}}\left\{\frac{d^{4} \tilde{v}}{d s^{4}}+\frac{1}{R_{S}{ }^{2}} \cdot \frac{d^{2} \tilde{v}}{d s^{2}}+\left(\rho_{y} \rho_{z}\right)^{2}\left(1-\frac{I_{\xi}{ }^{\prime}}{I_{\eta}{ }^{\prime}}\right) \tilde{v}\right\}-p_{i}{ }^{2} m A_{y} \tilde{v}-E_{s} I_{\eta}{ }^{\prime} \rho_{y} \rho_{z}\left(1-\frac{I_{\xi^{\prime}}}{I_{\eta}^{\prime}}\right) \cdot\left(\frac{d^{2} \widetilde{w}}{d s^{2}}+\rho_{y}{ }^{2} \widetilde{w}\right) \\
& +p_{i}{ }^{2} m A_{y z} \widetilde{w}-E_{s} I_{\eta}{ }^{\prime} \rho_{y}\left\{\frac{d^{2} \beta}{d s^{2}}+\left(\rho_{y}{ }^{2}+\rho_{z}{ }^{2} \frac{I_{\xi}{ }^{\prime}}{I_{\eta}}\right) \tilde{\beta}\right\}+p_{i}{ }^{2} m S_{y}{ }^{\prime} \tilde{\beta}=0 \\
& E_{s} I_{\xi^{\prime}}\left\{\frac{d^{4} \widetilde{w}}{d s^{4}}+\frac{1}{R_{S}{ }^{2}} \cdot \frac{d^{2} \widetilde{w}}{d s^{2}}+\left(\rho_{y} \rho_{z}\right)^{2}\left(1-\frac{I_{\eta}^{\prime}}{I_{\xi^{\prime}}^{\prime}}\right) \widetilde{w}\right\}-p_{i}{ }^{2} m A_{z} \widetilde{w}-E_{s} I_{\xi}^{\prime} \rho_{y} \rho_{z}\left(1-\frac{I_{\eta}^{\prime}}{I_{\xi^{\prime}}}\right) \cdot\left(\frac{d^{2} \widetilde{v}}{d s^{2}}+\rho_{z}{ }^{2} \tilde{v}\right) \\
& +p_{i}{ }^{2} m A_{y z}{ }^{\prime} \tilde{v}-E_{s} I_{\xi} \rho_{z}\left\{\frac{d^{2} \tilde{\beta}}{d s^{2}}+\left(\rho_{z}{ }^{2}+\rho_{y}{ }^{2} \frac{I_{\eta}{ }^{\prime}}{I_{\xi}{ }^{\prime}}\right) \tilde{\beta}\right\}-p_{i}{ }^{2} m S_{z}{ }^{\prime} \tilde{\beta}=0 \\
& E_{s} C_{w}\left\{\frac{d^{4} \tilde{\beta}}{d s^{4}}-\frac{G_{s} J}{E_{s} C_{w}} \cdot \frac{d^{2} \tilde{\beta}}{d s^{2}}+\left(\rho_{z^{2}} \frac{I_{\xi^{\prime}}}{C_{w}}+\rho_{y^{2}} \frac{I_{\eta}^{\prime}}{C_{w}}\right) \tilde{\beta}\right\}-p_{i}{ }^{2} m I_{S} \tilde{\beta} \\
& +E_{s} C_{w} \rho_{y}\left\{\frac{d^{4} \tilde{v}}{d s^{4}}-\left(\frac{G_{s} J}{E_{s} C_{w}}+\frac{I_{\eta}^{\prime}}{C_{w}}\right) \cdot \frac{d^{2} \tilde{v}}{d s^{2}}+\rho_{z}{ }^{2}\left(\frac{I_{\xi^{\prime}}}{C_{w}}-\frac{I_{\eta^{\prime}}{ }^{\prime}}{C_{w}}\right) \tilde{v}\right\}+p_{i}{ }^{2} m S_{y} \tilde{v} \\
& +E_{s} C_{w} \rho_{z}\left\{\frac{d^{4} \widetilde{w}}{d s^{4}}-\left(\frac{G_{s} J}{E_{s} C_{w}}+\frac{I_{\xi^{\prime}}{ }^{\prime}}{C_{w}}\right) \cdot \frac{d^{2} \widetilde{w}}{d s^{2}}+\rho_{y}{ }^{2}\left(\frac{I_{\eta}{ }^{\prime}}{C_{w}}-\frac{I_{\xi}^{\prime}}{C_{w}}\right) \widetilde{w}\right\}-p_{i} m S_{z} \widetilde{w}-=0
\end{aligned}
$$

The solutions for three shape functions $\tilde{v}, \widetilde{w}$ and $\tilde{\beta}$ may be represented by the following type of exponential functions

$$
K_{m} \exp \left(r_{m} s\right)
$$

The symbol $r_{m}$ is the roots of characteristic equation (52) in which unknown circular frequencies are involved. The symbol $K_{m}$ is constant of integration which can be determined by the boundary conditions imposed upon a curved girder bridge.

Concerning with the characteristic equation (52), the value $r_{m}$ should be determined as the roots of algebraric equation of the 12 th degree, which contains the twelve arbitrary constant of integration $K_{1}, K_{2}, \cdots, K_{12}$. If the roots $r_{m}$ are known, it is comparatively easy to solve the frequency equation in a simply supported curved girder bridge. In a continuous curved girder bridge, however, it is a difficult problem to find the exact solution, because at the middle supports there are many additional boundary conditions. Therefore, it appears to be not practical to determine the frequency by the above mentioned process.
Accordingly, let us now adopt the following approximate shape functions to find the frequency equation as simply as possible.

$$
\left.\begin{array}{c}
\tilde{v}(s)=\sum_{i=1,2, \cdots} \bar{v}_{i} \nu_{i}(s) \\
\widetilde{w}(s)=\sum_{i=1,2, \cdots} \bar{w}_{i} \omega_{i}(s) \\
\tilde{\beta}(s)=\sum_{i=1,2, \cdots}^{\sum} \bar{b}_{i} \mathfrak{b}_{i}(s)
\end{array}\right\}
$$

Here, the constants $\bar{v}_{i}, \bar{v}_{i}$ and $\bar{b}_{i}$ designate the amplitudes of bending vibration in the directions of the axes $S y, S z$ and of torsional vibration about the axis $S x$ respectively. Variables $\nu_{i}, \omega_{i}$ and $\mathfrak{b}_{i}$ are only the functions of curvilinear coordinates $s$. Obviously, they must satisfy all the geometrical and the mechanical boundary conditions imposed upon a curved girder bridge.

For example, if a continuous curved girder bridge have the middle supports at the section $s=L_{\mathrm{I}}, L_{\mathrm{II}}$, $\cdots, L_{R}, \cdots, L$, the boundary conditions at the section $s=L_{R}$ can be written as follows.

$$
\left[\nu_{i}\right]_{s=L_{R \pm 0}}=\left[\omega_{i}\right]_{s=L_{R \pm 0}}=\left[\mathfrak{b}_{i}\right]_{s=L_{R \pm 0}}=0
$$

Furthermore, the following conditions of continuity at the same section can be written,

$$
\begin{aligned}
& {\left[\frac{d \nu_{i}}{d s}\right]_{s=L_{R-0}}=\left[\frac{d \nu_{i}}{d s}\right]_{s=L_{R+0}},\left[\frac{d \omega_{i}}{d s}\right]_{s=L_{R-0}}=\left[\frac{d \omega_{i}}{d s}\right]_{s=L_{R+0}},\left[\frac{d \mathfrak{b}_{i}}{d s}\right]_{s=L_{R-0}}=\left[\frac{d \mathfrak{b}_{i}}{d s}\right]_{s=L_{R+0}}} \\
& {\left[\frac{d^{2} \nu_{i}}{d s^{2}}\right]_{s=L_{R-0}}=\left[\frac{d^{2} \nu_{i}}{d s^{2}}\right]_{s=L_{R+0}},\left[\frac{d^{2} \omega_{i}}{d s^{2}}\right]_{s=L_{R-0}}=\left[\frac{d^{2} \omega_{i}}{d s^{2}}\right]_{s=L_{R+0}},\left[\frac{d^{2} \mathfrak{b}_{i}}{d s^{2}}\right]_{s=L_{R-0}}=\left[\frac{d^{2} \mathfrak{b}_{i}}{d s^{2}}\right]_{s=L_{R+0}}}
\end{aligned}
$$


If this bridge is simply supported on both ends $s=0$ and $s=L$, the end conditions should be given in the following form,

$$
\left.\begin{array}{l}
{\left[\nu_{i}\right]_{s=0}=\left[\omega_{i}\right]_{s=0}=\left[\mathfrak{b}_{i}\right]_{s=0}=0} \\
{\left[\nu_{i}\right]_{s=L}=\left[\omega_{i}\right]_{s=L}=\left[\mathfrak{b}_{i}\right]_{s=L=0}}
\end{array}\right\}
$$

and

$$
\left.\begin{array}{l}
{\left[\frac{d^{2} \nu_{i}}{d s^{2}}\right]_{s=0}=\left[\frac{d^{2} \omega_{i}}{d s^{2}}\right]_{s=0}=\left[\frac{d^{2} \mathfrak{b}_{i}}{d s^{2}}\right]_{s=0}=0} \\
{\left[\frac{d^{2} \nu_{i}}{d s^{2}}\right]_{s=L}=\left[\frac{d^{2} \omega_{i}}{d s^{2}}\right]_{s=L}=\left[\frac{d^{2} \mathfrak{b}_{i}}{d s^{2}}\right]_{s=L}=0}
\end{array}\right\}
$$

In addition to the above conditions, every shape functions should be determined so as to satisfy the conditions of orthogonality; i.e., when $i \neq j(i=1,2$, $3, \cdots \cdots, j=1,2,3 \cdots \cdots)$

$$
\left.\begin{array}{l}
\int_{0}^{L} \Omega_{i} \nu_{j} d s=0, \quad \int_{0}^{L} \Omega_{i} \omega_{j} d s=0, \quad \int_{0}^{L} \Omega_{i} \mathfrak{b}_{j} d s=0 \\
\int_{0}^{L} \frac{d^{2} \Omega_{i}}{d s^{2}} \nu_{j} d s=0, \int_{0}^{L} \frac{d^{2} \Omega_{i}}{d s^{2}} \omega_{j} d s=0, \int_{0}^{L} \frac{d^{2} \Omega_{i}}{d s^{2}} \mathfrak{b}_{j} d s=0 \\
\int_{0}^{L} \frac{d^{4} \Omega_{i}}{d s^{4}} \nu_{j} d s=0, \int_{0}^{L} \frac{d^{4} \Omega_{i}}{d s^{4}} \omega_{j} d s=0, \int_{0}^{L} \frac{d^{4} \Omega_{i}}{d s^{4}} \mathfrak{b}_{j} d s=0
\end{array}\right\}
$$

where the symbol $\Omega_{i}$ is a representative of three quantities $\nu_{i}, \omega_{i}$ and $\mathfrak{b}_{i}$.

By substituting Eq. (54) into Eq. (52), we can find the ratio between $\bar{v}_{i}, \bar{w}_{i}$ and $\bar{b}_{i}$ corresponding to a minimum value of circular frequency. Applying Galerkin's method and considering the orthogonal conditions of Eq. (59) $)_{1 \sim 3}$, a set of linear simultaneous equations for unknown amplitudes $\bar{v}_{i}, \bar{w}_{i}$ and $\bar{b}_{i}$ can be obtained as follows;

$$
\begin{aligned}
& \left\{\left(p_{i, v v}^{2}-p_{i}{ }^{2}\right) A_{y} \int_{0}^{L} \nu_{i}{ }^{2} d s\right\} \bar{v}_{i}-\left\{\left(p_{i, w v}^{2}-p_{i}{ }^{2}\right) A_{y z} \int_{0}^{L} \nu_{i} w_{i} d s\right\} \bar{w}_{i}-\left\{\left(p_{i, \beta v}^{2}-p_{i}{ }^{2}\right) S_{y} \int_{0}^{L} \nu_{i} \mathfrak{b}_{i} d s\right\} \bar{b}_{i}=0 \\
- & \left\{\left(p_{i, v w}^{2}-p_{i}{ }^{2}\right) A_{y z} \int_{0}^{L} \nu_{i} w_{i} d s\right\} \bar{v}_{i}+\left\{\left(p_{i, w w}^{2}-p_{i}{ }^{2}\right) A z \int_{0}^{L} w_{i}{ }^{2} d s\right\} \bar{w}_{i}+\left\{\left(p_{i, \beta w}^{2}-p_{i}{ }^{2}\right) S_{z} \int_{0}^{L} w_{i} \mathfrak{b}_{i} d s\right\} \bar{b}_{i}=0 \\
- & \left\{\left(p_{i, v \beta}^{2}-p_{i}{ }^{2}\right) S_{y} \int_{0}^{L} \nu_{i} \mathfrak{b}_{i} d s\right\} \bar{v}_{i}+\left\{\left\{p_{i, w \beta}^{2}-p_{i}{ }^{2}\right) S_{z} \int_{0}^{L} w_{i} \mathfrak{b}_{i} d s\right\} \bar{w}_{i}+\left\{\left(p_{i, \beta \beta}^{2}-p_{i}{ }^{2}\right) I_{s} \int_{0}^{L} \mathfrak{b}_{i}{ }^{2} d s\right\} \bar{b}_{i}=0
\end{aligned}
$$

where the values $p_{i, v v}^{2}, p_{i, w w}^{2}, p_{i, \beta \beta}^{2}, \cdots$ are determined by the following formulae;

$$
\begin{aligned}
& p_{i, v v}^{2}=E_{s} I_{\eta}^{\prime}\left\{\int_{0}^{L} \frac{d^{4} \nu_{i}}{d s^{4}} \nu_{i} d s-\int_{0}^{L} \frac{1}{R_{S}{ }^{2}}\left(-\frac{d^{2} \nu_{i}}{d s^{2}}\right) \nu_{i} d s+\left(1-\frac{I_{\xi}^{\prime}}{I_{\eta}^{\prime}}\right) \int_{0}^{L}\left(\rho_{y} \rho_{z}\right)^{2} \nu_{i}{ }^{2} d s\right\} / m A_{y} \int_{0}^{L} \nu_{i}{ }^{2} d s \\
& p_{i, w v}^{2}=-E_{s} I_{\eta}^{\prime}\left(1-\frac{I_{\xi}^{\prime}}{I_{\eta}^{\prime}}\right)\left\{\int_{0}^{L} \rho_{y} \rho_{z}\left(-\frac{d^{2} \omega_{i}}{d s^{2}}\right) \nu_{i} d s-\int_{0}^{L} \rho_{y}{ }^{3} \rho_{z} \omega_{i} \nu_{i} d s\right\} / m A_{y z} \int_{0}^{L} \omega_{i} \nu_{i} d s \\
& p_{i, \beta v}^{2}=-E_{s} I_{\eta}{ }^{\prime}\left\{\int_{0}^{L} \rho_{y}\left(-\frac{d^{2} \mathfrak{b}_{i}}{d s^{2}}\right) \nu_{i} d s-\int_{0}^{L} \rho_{y}\left(\rho_{y}{ }^{2}+\rho_{z}{ }^{2} \frac{I_{\xi}^{\prime}}{I_{\eta^{\prime}}}\right) \mathfrak{b}_{i} \nu_{i} d s\right\} / m S_{y^{\prime}} \int_{0}^{L} \mathfrak{b}_{i} \nu_{i} d s \\
& p_{i, v w}^{2}=-E_{s} I_{\xi}^{\prime}\left(1-\frac{I_{\eta}^{\prime}}{I_{\xi}^{\prime}}\right)\left\{\int_{0}^{L} \rho_{y} \rho_{z}\left(-\frac{d^{2} \omega_{i}}{d s^{2}}\right) \nu_{i} d s-\int_{0}^{L} \rho_{y} \rho_{z}{ }^{3} \nu_{i} \omega_{i} d s\right\} / m A_{y z} \int_{0}^{L} \nu_{i} \omega_{i} d s \\
& p_{i, w w}^{2}=E_{s} I_{\xi}{ }^{\prime}\left\{\int_{0}^{L} \frac{d^{4} \omega_{i}}{d s^{4}} \omega_{i} d s-\int_{0}^{L} \frac{1}{R_{S}{ }^{2}}\left(-\frac{d^{2} \omega_{i}}{d s^{2}}\right) \omega_{i} d s+\left(1-\frac{I_{\eta}^{\prime}}{I_{\xi}^{\prime}}\right) \int_{0}^{L}\left(\rho_{y} \rho_{z}\right)^{2} \omega_{i}{ }^{2} d s\right\} / m A_{z} \int_{0}^{L} \omega_{i}{ }^{2} d s \\
& p_{i, \beta w}^{2}=E_{s} I_{\xi}^{\prime}\left\{\int_{0}^{L} \rho_{z}\left(-\frac{d^{2} \mathfrak{b}_{i}}{d s^{2}}\right) \omega_{i} d s-\int_{0}^{L} \rho_{z}\left(\rho_{z}{ }^{2}+\rho_{y^{2}} \frac{I_{\eta}^{\prime}}{I_{\xi}^{\prime}}\right) \mathfrak{b}_{i} \omega_{i} d s\right\} / m S_{z} \int_{0}^{L} \mathfrak{b}_{i} \omega_{i} d s \\
& p_{i, v \beta}^{2}=-E_{s} C_{w}\left\{\int_{0}^{L} \rho_{y} \frac{d^{4} \nu_{i}}{d s^{4}} \mathfrak{b}_{i} d s+\left(\frac{G_{s} J}{E_{s} C_{w}}+\frac{I_{\eta}^{\prime}}{C_{w}}\right) \int_{0}^{L} \rho_{y}\left(-\frac{d^{2} \nu_{i}}{d s^{2}}\right) \mathfrak{b}_{i} d s\right. \\
& \left.+\left(\frac{I_{\xi}^{\prime}-I_{\eta}{ }^{\prime}}{C_{w}}\right) \int_{0}^{L} \rho_{y} \rho_{z}{ }^{2} \nu_{i} \mathfrak{b}_{i} d s\right\} / m S_{y} \int_{0}^{L} \nu_{i} \mathfrak{b}_{i} d s \\
& p_{i, w \beta}^{2}=E_{s} C_{w}\left\{\int_{0}^{L} \rho_{z} \frac{d^{4} \omega_{i}}{d s^{4}} \mathfrak{b}_{i} d s+\left(\frac{G_{s} J}{E_{s} C_{w}}+\frac{I_{\xi}^{\prime}}{C_{w}}\right) \int_{0}^{L} \rho_{z}\left(-\frac{d^{2} \omega_{i}}{d s^{2}}\right) \mathfrak{b}_{i} d s\right. \\
& \left.+\left(\frac{I_{\eta}^{\prime}-I_{\xi}^{\prime}}{C_{w}}\right) \int_{0}^{L} \rho_{y}^{2} \rho_{z} \omega_{i} \mathfrak{b}_{i} d s\right\} / m S_{z} \int_{0}^{L} \omega_{i} \mathfrak{b}_{i} d s \\
& p_{i, \beta \beta}^{2}=E_{s} C_{w}\left\{\int_{0}^{L} \frac{d^{4} \mathfrak{b}_{i}}{d s^{4}} \mathfrak{b}_{i} d s+\frac{G_{s} J}{E_{s} C_{w}} \int_{0}^{L}\left(-\frac{d^{2} \mathfrak{b}_{i}}{d s^{2}}\right) \mathfrak{b}_{i} d s+\int_{0}^{L}\left(\rho_{y^{2}} \frac{I_{\xi}^{\prime}}{C_{w}}+\rho_{z}{ }^{2} \frac{I_{\eta}{ }^{\prime}}{C_{w}}\right) \mathfrak{b}_{i}{ }^{2} d s\right\} / m I_{s} \int_{0}^{L} \mathfrak{b}_{i}{ }^{2} d s
\end{aligned}
$$

and also they represent the circular frequencies corresponding to each individual vibration.

In order that every amplitude of three kinds of

modes have a certain value other than zero, the determinant containing the coefficients as the elements must be equal to zero. Thus

$$
\begin{array}{ccc}
\left(p_{i, v v}^{2}-p_{i}{ }^{2}\right) A_{y} \int_{0}^{L} \nu_{i}{ }^{2} d s & -\left(p_{i, w v}^{2}-p_{i}{ }^{2}\right) A_{y z} \int_{0}^{L} \nu_{i} \omega_{i} d s & -\left(p_{i, \beta v}^{2}-p_{i}{ }^{2}\right) S_{y}{ }^{\prime} \int_{0}^{L} \nu_{i} \mathfrak{b}_{i} d s \\
-\left(p_{i, v w}^{2}-p_{i}{ }^{2}\right) A_{y z} \int_{0}^{L} \nu_{i} \omega_{i} d s & \left(p_{i, w w}^{2}-p_{i}{ }^{2}\right) A_{z} \int_{0}^{L} \omega_{i}{ }^{2} d s & \left(p_{i, \beta w}{ }^{2}-p_{i}{ }^{2}\right) S_{z} \int_{0}^{L} \omega_{i} \mathfrak{b}_{i} d s \\
-\left(p_{i, v \beta}^{2}-p_{i}{ }^{2}\right) S_{y} \int_{0}^{L} \nu_{i} \mathfrak{b}_{i} d s & \left(p_{i, w \beta}^{2}-p_{i}{ }^{2}\right) S_{z} \int_{0}^{L} \omega_{i} \mathfrak{b}_{i} d s & \left(p_{i, \beta \beta}{ }^{2}-p_{i}{ }^{2}\right) I_{s} \int_{0}^{L} \mathfrak{b}_{i}{ }^{2} d s
\end{array}
$$


In general, the form of individual shape function is more or less different from every other. Nevertheless, it is pointed out in reference (5) that the common shape functions may be used approximately for them when they obey the identical boundary conditions as given by Eq. (55) to (58). So, putting

$$
\nu_{i}=w_{i}=\mathfrak{b}_{i}=\Omega_{i}
$$

Eq. (62) can be simplified as follows;

$$
\left|\begin{array}{ccc}
\left(p_{i, v v}^{2}-p_{i}{ }^{2}\right) A_{y} & -\left(p_{i, w v}^{2}-p_{i}{ }^{2}\right) A_{y z} & -\left(p_{i, \beta v}^{2}-p_{i}{ }^{2}\right) S_{y} \\
-\left(p_{i, v w}^{2}-p_{i}{ }^{2}\right) A_{y z} & \left(p_{i, w w}^{2}-p_{i}{ }^{2}\right) A_{z} & \left(p_{i, \beta w}^{2}-p_{i}{ }^{2}\right) S_{z} \\
-\left(p_{i, v \beta}^{2}-p_{i}{ }^{2}\right) S_{y} & \left(p_{i, w \beta}^{2}-p_{i}{ }^{2}\right) S_{z} & \left(p_{i, \beta \beta}-p_{i}{ }^{2}\right) I_{s}
\end{array}\right|=0
$$

This is an approximate form of the frequency equation for determining the circular frequencies $p_{i}$ of a curved girder bridge having general crosssection. After the solutions for $p_{i}$ are obtained, by substituting those values into $\mathrm{Eq}$. (60), the ratio between amplitudes $\bar{v}_{i}, \bar{w}_{i}$ and $\bar{b}_{i}$ will readily be given. Thus, the patterns of the vibrations will be made clear.

Finally, as is seen from Eq. (44) to (47), (61), and (64), the values $p_{i}$ are governed by the geometrical properties of the structure, namely, the quantities $L, \Phi, R_{S}, A, S, I, J$ and $C_{w}$. So, it is meaningful to discuss the effects of these values concretely, and also introduce some special cases of frequency equations.

\section{Approximate Solution of Frequency Equa- tion.}

(1) Plane shape and cross sectional quantities.

The frequency equation has been derived on an assumption that a curved girder bridge has a constant radius of curvature and a uniform crosssection. If these quantities vary continuously along the axial direction, it will become much difficult to solve the fundamental equation of motion.

However, the radius of curvature of the usual continuous curved girder bridge may be approximately assumed to be constant in each span as shown in Fig. 8, where the radius of curvature in each span are denoted by $R_{S, 1}, R_{S, 2}, \cdots, R_{S, r}, \cdots, R_{S, n}$, the central angle by $\Phi_{1}, \Phi_{2}, \cdots, \Phi_{r}, \cdots, \Phi_{n}$, and also the span length by $L_{1}, L_{2}, \cdots, L_{r}, \cdots, L_{n}$. The sub-

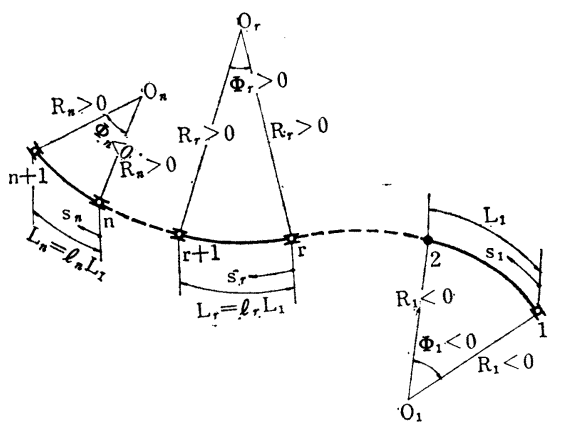

Fig. 8 Plane shape of curved girder bridge. coordinates $s_{r}$ having the origin at the support $r$ will be used properly as the curvilinear coordinates in the $r$ th span. The sign of quantities $R_{S, r}$ and $\Phi_{r}$ are positive, if in the $r$ th span the girder is bent toward the right side as we proceed from the origin $s_{r}=0$ along the girder axis in the direction of coordinate $s$.

Strictly speaking, the cross-section of an ordinary curved girder bridge varies somewhat, so the mean values of all the cross sectional quantities may be adopted practically. Because the problem of free vibration is not a microscopic one of investigating the local deformation or the stress distribution, but a macroscopic one of clarifying the dynamical behaviour of a whole bridge. Considering these situation, it is to be expected that the errors included in the approximate solution for frequency will not be serious even in the extreme case of girder bridge with varing height ${ }^{14)}$.

Accordingly, when a symbol $Q_{W, r}\left(s_{r}\right)$ represents all the varing sectional quantities in the $r$ th span, the mean values $\bar{Q}_{W}$ of $Q_{W, r}\left(s_{r}\right)$ may be given by,

$$
\bar{Q}_{W, r}=\frac{1}{L_{r}} \int_{0}^{L_{r}} Q_{W, r}\left(s_{r}\right) d s_{r}
$$

Than, the mean value $Q_{W}{ }^{*}$ all over the bridge can be found by

$$
Q_{W} *=\sum_{r=1}^{n} l_{r} \bar{Q}_{W, r} / \sum_{r=1}^{n} l_{r}
$$

Here, the weight $l_{r}$ is the ratio between the $r$ th span and the reference span. Namely, we put

$$
l_{1}=1, l_{2}=L_{2} / L_{1}, \cdots \cdots, l_{r}=L_{r} / L_{1}, l_{n}=L_{n} / L_{1}
$$

Thus, the mean values $I_{G}{ }^{*}, J^{*}$ and $C_{w}{ }^{*}$ can directly decided by Eq. (65) and (66).

However, as to the cross sectional area $A_{s}$ of a usual bridge, we must take into account not only those of the main girder but also of the slab and the secondary members such as floor beams, diaphrams and so on, since the area of the latter is usually not much smaller than that of the former. Therefore, the cross sectional area of the bridge may be replaced by the dead load intensity $w_{d, r}$ per unit length, namely,

$$
A_{s, r}\left(s_{r}\right)=w_{d, r}\left(s_{r}\right) / r_{s}
$$

Then, by using Eq. (65), the mean value of the $r$ 
th span can easily be found.

On the other hand, since the radius of curvature $R_{S}, R_{O}$ or $R_{G}$ should be included in the formulae for the mean values $m^{*}, I_{\xi}{ }^{*}, I_{\eta}{ }^{*}$ as is seen in Eq. (44) and (28), they have to be determined from following equations instead of Eq. (66).

$$
\begin{aligned}
& m^{*}=\gamma_{s} \sum_{r=1}^{n} \frac{R_{G, r}}{R_{S, r}} \cdot l_{r} / g \sum_{r=1}^{n} l_{r} \ldots \ldots . \\
& I^{\prime *}{ }_{\xi \text { or } r}=\sum_{r=1}^{n} \frac{R_{S, r}}{R_{O, r}} I_{\xi \text { or } \eta} \cdot l_{r} / \sum_{r=1}^{n} l_{r}
\end{aligned}
$$

Furthermore, the mean polar moment of inertia $I_{S}{ }^{*}$ with respect to the shear center may be written as follows from Eq. (45).

$$
\begin{aligned}
I_{S} *= & \sum_{r=1}^{n} \frac{l_{r}}{L_{r}} \int_{0}^{L_{r}}\left\{I_{G, r}\left(s_{r}\right)+A_{s, r}\left(s_{r}\right) \cdot y_{G, r}{ }^{2}\left(s_{r}\right)\right. \\
& \left.+A_{s, r}\left(s_{r}\right) \cdot z_{G, r}{ }^{2}\left(s_{r}\right)\right\} d s_{r} / \sum_{r=1}^{n} l_{r}
\end{aligned}
$$

If the mean values of each span are used, $I_{S}{ }^{*}$ may be written approximately,

$$
I_{S} *=I_{G} *+\sum_{r=1}^{n}\left\{\bar{A}_{s, r}\left(\bar{y}_{G, r^{2}}+\bar{z}_{G, r^{2}}\right)\right\} l_{r} / \sum_{r=1}^{n} l_{r}
$$

In the similar way, the mean values $A^{*}$ and $S^{*}$ may be given from Eq. (5), (46) and (47).

$$
\left.\begin{array}{l}
A_{y} *=\sum_{r=1}^{n} \bar{A}_{s, r}\left(1+\frac{\bar{z}_{G, r}}{R_{S, r}} \sin \bar{\alpha}_{r}\right) l_{r} / \sum_{r=1}^{n} l_{r} \\
A_{z} *=\sum_{r=1}^{n} \bar{A}_{s, r}\left(1-\frac{\bar{y}_{G, r}}{R_{S, r}} \cos \bar{\alpha}_{r}\right) l_{r} / \sum_{r=1}^{n} l_{r} \\
A_{y z}{ }^{*}=\sum_{r=1}^{n}\left( \pm \bar{A}_{s, r} \frac{\bar{y}_{G, r}}{R_{S, r}} \sin \bar{\alpha}_{r}\right) l_{r} / \sum_{r=1}^{n} l_{r} \\
A_{y z}{ }^{*}=-\sum_{r=1}^{n}\left( \pm \bar{A}_{s, r} \frac{\bar{z}_{G, r}}{R_{S, r}} \cos \bar{\alpha}_{r}\right) l_{r} / \sum_{r=1}^{n} l_{r}
\end{array}\right\}
$$

and

$$
\begin{aligned}
& S_{y}^{\prime *}=\sum_{r=1}^{n}\left(\bar{A}_{s, r} \bar{z}_{G, r}+\frac{\bar{I}_{S, r}}{R_{S, r}} \sin \bar{\alpha}_{r}\right) l_{r} / \sum_{r=1}^{n} l_{r} \\
& S_{y} *=\sum_{r=1}^{n} \bar{A}_{s, r} \bar{z}_{G, r} l_{r} / \sum_{r=1}^{n} l_{r} \\
& S_{z}{ }^{*}=\sum_{r=1}^{n}\left\{ \pm\left(\bar{A}_{s, r} \bar{y}_{G, r}-\frac{\bar{I}_{S, r}}{R_{S, r}} \cos \bar{\alpha}_{r}\right)\right\} l_{r} / \sum_{r=1}^{n} l_{r} \\
& S_{z}^{*}=\sum_{r=1}^{n} \pm\left(\bar{A}_{s, r} \bar{y}_{G, r}\right) l_{r} / \sum_{r=1}^{n} l_{r}
\end{aligned}
$$

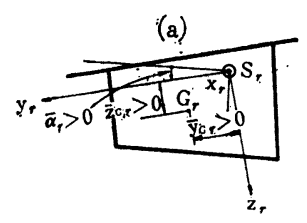

(b)

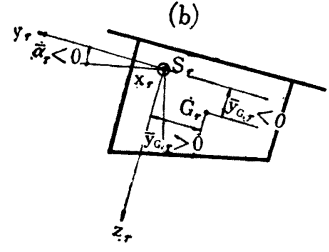

Fig. 9 Cross-section of curved girder bridge.
The signs of $\bar{y}_{G, r}, \bar{z}_{G, r}, \bar{\alpha}_{r}$ in Eq. (72) and (73) are illustrated in Fig. 9, in which two cross-section (a) and (b) are completely same in shape and size, but these bridges are bent toward in the opposite direction to each other. Therefore, if the positive direction of rectangular coordinates $s_{r}$ in Eq. (60) is defined to be coincided with the positive direction of coordinate $s$, the coordinate $\bar{y}_{G, r}$, of $G_{r}$ and the angle $\bar{\alpha}_{r}$ of the inclination of neutral axes. must have the negative signs in Fig. 9 (b).

Consequently, the double signs in Eq. (72) and (73) must be positive (or negative), if $R_{S, r}$ is positive (or negative).

\section{(2) Approximate solution of shape function.}

Let us consider the shape function for the $r$ th span of a curved girder bridge shown in Fig. 8 .

The approximate shape function analogous to Eq. (53) may be used as the solution of our problem, but all the boundary conditions and also the conditions of orthogonality should be satisfied by theseshape function. So, the following function will be adopted approximately,

$$
\begin{aligned}
\nu_{i, r} & =\omega_{i, r}=\mathfrak{b}_{i, r}=\Omega_{i, r} \\
& =\boldsymbol{K}_{i, r} \sin k_{i} s_{r}+\boldsymbol{\Lambda}_{i, r} \cos k_{i} s_{r} \\
& +\boldsymbol{M}_{i, r} \sinh k_{i} s_{r}+\boldsymbol{N}_{i, r} \cosh k_{i} s_{r}
\end{aligned}
$$

where the symbol $k_{i}$ is a parameter concerned with the span ratio $l_{r}$, and the symbols $\boldsymbol{K}_{i, r}, \boldsymbol{\Lambda}_{i, r}, \boldsymbol{M}_{i, r}$, $\boldsymbol{N}_{i, r}$ are coefficients determined by the boundary conditions.

From Eq. (55) to (58), the boundary conditions. are rewritten corresponding to Fig. 8,

$$
\left[\Omega_{i, r}\right]_{s_{r=0}}=\left[\Omega_{i, r}\right] s_{r=} L_{r}=0
$$

at all the supports including the end supports $r=1$. and $r=n+1$. The conditions of continuity at the: $r+1$ th support should be written as follows,

$$
\left.\begin{array}{l}
{\left[\frac{d \Omega_{i, r}}{d s_{r}}\right]_{s_{r=} L_{r}}=\left[\frac{d \Omega_{i, r+1}}{d s_{r+1}}\right]_{s_{r+1=0}}} \\
{\left[\frac{d^{2} \Omega_{i, r}}{d s_{r}{ }^{2}}\right]_{s_{r=L_{r}}}=\left[\frac{d^{2} \Omega_{i, r+1}}{d s_{r+1}{ }^{2}}\right]_{s_{r+1}=0}}
\end{array}\right\}
$$

And the conditions at both ends $r=1$ and $r=n+1$ are

$$
\left[\frac{d^{2} \Omega_{i, 1}}{d s_{1}{ }^{2}}\right]_{s_{1=0}}=\left[\frac{d^{2} \Omega_{i, n}}{d s_{n}{ }^{2}}\right]_{s_{n}=L_{n}}=0
$$

From these Eq. (74) to (77), the following approximate shape functions can be obtained for the two. cases.

a) Simply supported girder bridge.

In a simply supported girder bridge, we can put $\boldsymbol{n}=1$ and $\boldsymbol{\Lambda}_{\boldsymbol{i}, 1}=\boldsymbol{M}_{\boldsymbol{i}, 1}=\boldsymbol{N}_{\boldsymbol{i}, 1}=0$, so that

$$
\Omega_{i, 1}=K_{i, 1} \sin k_{i} s_{1}
$$

where

$$
k_{i} L_{1}=i \pi(i=1,2,3, \cdots \cdots), \quad \boldsymbol{K}_{i, 1}=1
$$


b) Continuous girder bridge.

The shape function of the $r$ th span may be given approximately by the following equation ${ }^{15)}$.

$$
\begin{aligned}
\Omega_{i, r}= & \Lambda_{i, r}\left[\frac{\sin \left\{k_{i}\left(L_{r}-s_{r}\right)\right\}}{\sin k_{i} L_{r}}-\frac{\sinh \left\{k_{i}\left(L_{r}-s_{r}\right)\right\}}{\sinh k_{i} L_{r}}\right] \\
& +\Lambda_{i, r+1}\left[\frac{\sin k_{i} s_{r}}{\sin k_{i} L_{r}}-\frac{\sinh k_{i} s_{r}}{\sinh k_{i} L_{r}}\right] \ldots \ldots \ldots \ldots(80)
\end{aligned}
$$

It is clear that all the boundary conditions (75) to (77) are satisfied by the above function. Herein, the shape parameters $k_{i}(i=1,2,3, \cdots)$ can be decided by solving the following $(n-1)$ simultaneous equations.

$$
\begin{aligned}
\Lambda_{i, r} l_{r} \Psi_{i, r} & +\Lambda_{i, r+1}\left(l_{r} \chi_{i, r}+l_{r+1} \chi_{i, r+1}\right) \\
& +\Lambda_{i, r+2} l_{r+1} \Psi_{i, r+1}=0 \cdots \cdots \cdots \\
& (r=1,2,3, \cdots, n-1)
\end{aligned}
$$

From the end conditions (77),

$$
\Lambda_{i, 1}=\Lambda_{i, n+1}=0
$$

The variables $\Psi_{i, r}$ and $\chi_{i, r}$ are the function of $l_{r} k_{i} L_{1}$. (see Appendix)

$$
\left.\begin{array}{rl}
\Psi_{i, r} & =\frac{1}{l_{r} k_{i} L_{1}} \cdot\left(\operatorname{cosec} l_{r} k_{i} L_{1}-\operatorname{cosech} l_{r} k_{i} L_{1}\right) \\
\chi_{i, r} & =\frac{1}{l_{r} k_{i} L_{1}} \cdot\left(\operatorname{coth} l_{r} k_{i} L_{1}-\cot l_{r} k_{i} L_{1}\right)
\end{array}\right\}
$$

As Eq. (81) is the linear homogeneous equations, a transcendental equation $f\left(k_{i} L_{1}\right)=0$ is obtained by putting the determinant built by the coefficients of $\Lambda_{i, 2}, \Lambda_{i, 3}, \cdots, \Lambda_{i, r}, \cdots, \Lambda_{i, n}$ equal to zero. After the roots $k_{i} L_{1}$ have been determined, the ratio among $\Lambda_{i, 2}, \Lambda_{i, 3}, \cdots, \Lambda_{i, r}, \cdots, \Lambda_{i, n}$ can be decided by substituting them again into Eq. (81). So, denoting this ratio by the symbol $\lambda_{i, r}$,

$$
\begin{gathered}
\lambda_{i, 1}=0, \lambda_{i, 2}=1, \cdots, \lambda_{i, r}=\Lambda_{i, r} / \Lambda_{i, 2}, \cdots, \lambda_{i, n+1}=0 \quad \text { bridge, it can be found } \\
k_{i} L_{1}=(2 i-1) \pi \\
=3.927,7.069, \cdots,\left(\frac{i}{2}+0.25\right) \pi ; \text { for } 2 \text { nd, } 4 \mathrm{th}, \cdots, 2 i \text { th mode }
\end{gathered}
$$

Now, the trial and error method is suitable to determine the roots $k_{i} L_{1}$, but it requires much labour in numerical calculation. However, when the variables $\Psi_{i, r}$ and $x_{i, r}$ are given in the form of table (see Appendix), it is comparatively easy to find the first approximate solution $\left[k_{i} L_{1}\right]_{1}$. When more exact values $\left[k_{i} L_{1}\right]_{2},\left[k_{i} L_{1}\right]_{3}, \cdots,\left[k_{i} L_{1}\right]_{h}, \cdots$ are needed, we can obtain it by the following formula.

$$
\left[k_{i} L_{1}\right]_{h+1}=\left[k_{i} L_{1}\right]_{h}+\left[\Delta k_{i} L_{1}\right]_{h}
$$

Hence the notation $\left[\Delta k_{i} L_{1}\right]_{h}$ is a compensating value for making the value $\left[k_{i} L_{1}\right]_{h}$ more accurate, it can be estimated on the basis of Newton's method.

$$
\left[\Delta k_{i} L_{1}\right]_{h}=-\left[f\left(k_{i} L_{1}\right)\right]_{h} /\left[\frac{d f\left(k_{i} L_{1}\right)}{d\left(k_{i} L_{1}\right)}\right]_{h}
$$

where the derivatives

$$
d \Psi_{i, r} / d\left(k_{i} L_{1}\right)=\Psi_{i, r^{\prime}} \text { and } d \chi_{i, r} / d\left(k_{i} L_{1}\right)=\chi_{i, r^{\prime}}
$$

involved in the function

$$
d f\left(k_{i} L_{1}\right) / d\left(k_{i} L_{1}\right)
$$

can easily be found by applying the following formulae;

$$
\left.\begin{array}{l}
\Psi_{i, r^{\prime}}=\frac{\zeta_{i, r}}{l_{r}}-\frac{\Psi_{i, r}}{k_{i} L_{1}} \\
\chi_{i, r^{\prime}}=k_{i} L_{1} \Psi_{i, r} \varphi_{i, r}-\frac{\chi_{i, r}}{k_{i} L_{1}}
\end{array}\right\}
$$

where new variables $\zeta_{i, r}$ and $\varphi_{i, r}$ are both the function of $l_{r} k_{i} L_{1}$. (see Appendix)

$$
\left.\begin{array}{rl}
\zeta_{i, r}= & \operatorname{cosech} l_{r} k_{i} L_{1} \operatorname{coth} l_{r} k_{i} L_{1} \\
& -\operatorname{cosec} l_{r} k_{i} L_{1} \cot l_{r} k_{i} L_{1} \\
\varphi_{i, r}= & \operatorname{cosec} l_{r} k_{i} L_{1}+\operatorname{cosech} l_{r} k_{i} L_{1}
\end{array}\right\}
$$

Especially, in a two equal span continuous girder bridge, it can be found that the values $k_{i} L_{1}$ are
Accordingly, the odd modes are the same as in simply supported girder bridge and are asymmetric, while the even modes are symmetric.

(3) Values $\boldsymbol{p}_{i, v v}^{2}, \underset{\boldsymbol{p}_{i, w w}}{2}, \boldsymbol{p}_{i, \beta \beta}^{2}, \cdots$, etc.

Before estimating the values ${p_{i, v v}}^{2}, p_{i, w w}^{2}, p_{i, \beta \beta}^{2}, \cdots$, etc., it needs to inquire whether the approximate shape function, obtained in (2), will satisfy the conditions of orthogonality or not.

First, in a simply supported girder bridge, it is clear that all conditions (59) ${ }_{1 \sim 3}$ are satisfied by them. Therefore, the shape function (78) gives a set of exact solutions.

Next, in a continuous girder bridge, differentiating $\Omega_{i, r}$ in Eq. (80) twice and four times with respect to $s_{r}$,

$$
\begin{aligned}
& \frac{d^{2} \Omega_{i, r}}{d s_{r}{ }^{2}}=-k_{i}{ }^{2}\left(\lambda_{i, r}\left[\frac{\sin \left\{k_{i}\left(L_{r}-s_{r}\right)\right\}}{\sin k_{i} L_{r}}+\frac{\sinh \left\{k_{i}\left(L_{r}-s_{r}\right)\right\}}{\sinh k_{i} L_{r}}\right]\right. \\
& \left.+\lambda_{i, r+1}\left[\frac{\sin k_{i} s_{r}}{\sin k_{i} L_{r}}+\frac{\sinh k_{i} s_{r}}{\sinh k_{i} L_{r}}\right]\right) \\
& \frac{d^{4} \Omega_{i, r}}{d s_{r}{ }^{4}}=k_{i}^{4} \Omega_{i, r}
\end{aligned}
$$

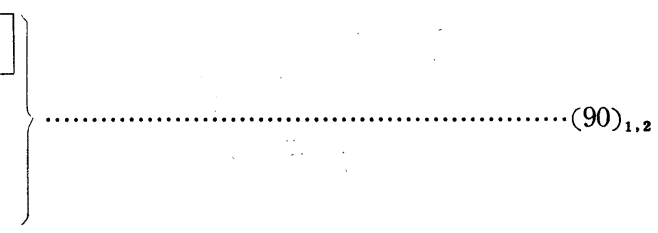

the following equation will be introduced by making use of the integration by parts. 


$$
\begin{aligned}
& \int_{0}^{L} \frac{d^{4} \Omega_{i}}{d s^{4}} \Omega_{j} d s=\sum_{r=1}^{n}\left[\frac{d^{3} \Omega_{i, r}}{d s_{r}{ }^{3}} \Omega_{j, r}-\frac{d^{3} \Omega_{j, r}}{d s_{r}{ }^{3}} \Omega_{i, r}\right]_{0}^{L_{r}} \\
& -\sum_{r=1}^{n-1}\left(\left\{\left[\frac{d^{2} \Omega_{i, r}}{d s_{r}{ }^{2}} \cdot \frac{d \Omega_{j, r}}{d s_{r}}\right]_{s_{r=} L_{r}}-\left[\frac{d^{2} \Omega_{i, r+1}}{d s_{r+1}^{2}} \cdot \frac{d \Omega_{j, r+1}}{d s_{r+1}}\right]_{s_{r+1=0}}\right\}-\left\{\left[\frac{d^{2} \Omega_{j, r}}{d s_{r}{ }^{2}} \cdot \frac{d \Omega_{i, r}}{d s_{r}}\right]_{s_{r=} L_{r}}\right.\right. \\
& \left.\left.-\left[\frac{d^{2} \Omega_{j, r+1}}{d s_{r+1}^{2}} \cdot \frac{d \Omega_{i, r+1}}{d s_{r+1}}\right]_{s_{r+1=0}}\right\}\right)-\left(\left\{\left[\frac{d^{2} \Omega_{i, 1}}{d s_{1}{ }^{2}} \cdot \frac{d \Omega_{j, 1}}{d s_{1}}\right]_{s_{1=0}}-\left[\frac{d^{2} \Omega_{i, n}}{d s_{n}{ }^{2}} \cdot \frac{d \Omega_{j, n}}{d s_{n}}\right]_{s_{n=} L_{n}}\right\}\right. \\
& \left.-\left\{\left[\frac{d^{2} \Omega_{j, 1}}{d s_{1}{ }^{2}} \cdot \frac{d \Omega_{i, 1}}{d s_{1}}\right]_{s_{1=0}}-\left[\frac{d^{2} \Omega_{j, n}}{d s_{n}{ }^{2}} \cdot \frac{d \Omega_{i, n}}{d s_{n}}\right]_{s_{n}=L_{n}}\right\}\right) \\
& +\int_{0}^{L} \frac{d^{4} \Omega_{j}}{d s^{4}} \Omega_{i} d s
\end{aligned}
$$

The first term on the right-hand side of Eq. (91) vanishes from the boundary conditions (75), the second from the conditions of continuity (76) and the third from the end conditions (77). Therefore, when Eq. $(90)_{2}$ is substituted into Eq. (91)

$$
k_{i}{ }^{4} \int_{0}^{L} \Omega_{i} \Omega_{j} d s=k_{j}{ }^{4} \int_{0}^{L} \Omega_{j} \Omega_{i} d s
$$

is obtained. For $i \neq j$ with $k_{i} \neq k_{j}$, the relationship of orthogonality

$$
\begin{aligned}
& \int_{0}^{L} \Omega_{i} \Omega_{j} d s=0 \\
& \int_{0}^{L} \frac{d^{4} \Omega_{i}}{d s^{4}} \Omega_{j} d s=0
\end{aligned}
$$

can be obtained.

Similarly,

$$
\begin{aligned}
\int_{0}^{L} \frac{d^{2} \Omega_{i}}{d s^{2}} \Omega_{j} d s= & \sum_{r=1}^{n}\left[\frac{d \Omega_{i, r}}{d s_{r}} \Omega_{j, r}-\frac{d \Omega_{j, r}}{d s_{r}} \Omega_{i, r}\right]_{0}^{L_{r}} \\
& +\int_{0}^{L} \frac{d^{2} \Omega_{j}}{d s^{2}} \Omega_{i} d s \ldots \ldots \ldots \ldots \ldots(92)
\end{aligned}
$$

is given, but the first term on the right-hand side must be equal to zero by the boundary conditions (75). Consequently,

$$
\int_{0}^{L} \frac{d^{2} \Omega_{i}}{d s^{2}} \Omega_{j} d s=\int_{0}^{L} \frac{d^{2} \Omega_{j}}{d s^{2}} \Omega_{i} d s .
$$

However, it is evident that another condition $(59)_{2}$ can not be satisfied even if Eq. (90), is substituted into the above equation.

In order to satisfy this condition perfectly, the shape function should take the same form as Eq. (53). Though the above approximate function $\Omega_{i}$ is quite proper for the conditions of orthogonality $(59)_{1,3}$, the errors will be produced in the remaining condition (59) ${ }_{2}$. However, it is important for structural engineering purposes to examine the lowest several modes of vibration. Accordingly, this paper deals with only the lowest several frequencies for the sake of simplicity.

Finally, by the above mentioned simplification, the values $p_{i, v v}^{2}, \underset{p_{i, w w}}{2}, p_{i, \beta \beta}^{2}, \cdots$, etc., contained in the frequency equation, may be obtained from Eq. (61) as follows;

$$
\begin{aligned}
& p_{i, v v}^{2}=\frac{E_{s} I_{\eta^{*}}{ }^{*}}{m^{*} A_{y^{*} L_{1}^{4}}}\left\{\left(k_{i} L_{1}\right)^{4}-\left(k_{i} L_{1}\right)^{2} \frac{\sum_{r=1}^{n}\left(\frac{\Phi_{r}}{l_{r}}\right)^{2} \Delta_{i, r}}{\sum_{r=1}^{n} \nabla_{i, r}}+\frac{1}{4}\left(1-\frac{I_{\xi}^{*} *}{I_{\eta}^{\prime *}}\right) \frac{\sum_{r=1}^{n}\left(\frac{\Phi_{r}}{l_{r}}\right)^{4} \nabla_{i, r} \sin ^{2} 2 \bar{\alpha}_{r}}{\sum_{r=1}^{n} \nabla_{i, r}}\right\} \\
& p_{i, w v}^{2}=-\frac{E_{s} I_{\eta}^{\prime *}}{2 m^{*} A_{y z}{ }^{*} L_{1}^{4}}\left(1-\frac{I_{\xi^{\prime}} *}{I_{\eta}{ }^{\prime *}}\right)\left\{\left(k_{i} L_{1}\right)^{2} \frac{\sum_{r=1}^{n}\left(\frac{\Phi_{r}}{l_{r}}\right)^{2} \Delta_{i, r} \sin \left( \pm 2 \bar{\alpha}_{r}\right)}{\sum_{r=1}^{n} \nabla_{i, r}}-\frac{\sum_{r=1}^{n}\left(\frac{\Phi_{r}}{l_{r}}\right)^{4} \nabla_{i, r} \sin ^{2} \bar{\alpha}_{r} \sin \left( \pm 2 \bar{\alpha}_{r}\right)}{\sum_{r=1}^{n} \nabla_{i, r}}\right\} \\
& p_{i, \beta v}^{2}=-\frac{E_{s} I_{\eta}^{\prime *}}{m^{*} S_{y}^{\prime *} L_{1}{ }^{\prime}}\left\{\left(k_{i} L_{1}\right)^{2} \frac{\sum_{r=1}^{n} \bar{\Phi}_{r} \Delta_{i, r} \sin \bar{\alpha}_{r}}{\sum_{r=1}^{n} \nabla_{i, r}}-\frac{\sum_{r=1}^{n}\left(\frac{\Phi_{r}}{l_{r}}\right)^{3} \nabla_{i, r} \sin \bar{\alpha}_{r}\left(\sin ^{2} \bar{\alpha}_{r}+\frac{I_{\xi}^{\prime *}}{I_{\eta}^{\prime *}} \cos ^{2} \bar{\alpha}_{r}\right)}{\sum_{r=1}^{n} \nabla_{i, r}}\right\} \\
& \left.p_{i, v w}^{2}=-\frac{E_{s} I_{\xi}{ }^{*}}{2 m^{*} A_{y z^{\prime} L_{1}}}\left(1-\frac{I_{\eta}{ }^{\prime *}}{I_{\xi}^{\prime *}}\right)\left(k_{i} L_{1}\right)^{2} \frac{\sum_{r=1}^{n}\left(\frac{\Phi_{r}}{l_{r}}\right)^{2} \Delta_{i, r} \sin \left( \pm 2 \bar{\alpha}_{r}\right)}{\sum_{r=1}^{n} \nabla_{i, r}}-\frac{\sum_{r=1}^{n}\left(\frac{\Phi_{r}}{l_{r}}\right)^{4} \nabla_{i, r} \cos ^{2} \bar{\alpha}_{r} \sin \left( \pm 2 \bar{\alpha}_{r}\right)}{\sum_{r=1}^{n} \nabla_{i, r}}\right\} \\
& \left.p_{i, w w}^{2}=\frac{E_{s} I_{\xi^{\prime}} *}{m^{*} A_{z^{*}} L_{1}^{4}}\left(k_{i} L_{1}\right)^{4}-\left(k_{i} L_{1}\right)^{2} \frac{\sum_{r=1}^{n}\left(\frac{\Phi_{r}}{l_{r}}\right)^{2} \Delta_{i, r}}{\sum_{r=1}^{n} \nabla_{i, r}}+\frac{1}{4}\left(1-\frac{I_{\eta}{ }^{\prime *}}{I_{\xi}^{\prime * *}}\right) \frac{\sum_{r=1}^{n}\left(\frac{\Phi_{r}}{l_{r}}\right)^{4} \nabla_{i, r} \sin ^{2} 2 \bar{\alpha}_{r}}{\sum_{r=1}^{n} \nabla_{i, r}}\right\} \\
& p_{i, \beta \omega}^{2}=\frac{E_{s} I_{\xi}^{\prime *}}{m^{*} S_{z^{\prime}} L_{1}{ }^{3}}\left\{\left(k_{i} L_{1}\right)^{2} \frac{\sum_{r=1}^{n}\left( \pm \frac{\Phi_{r}}{l_{r}}\right) \Delta_{i, r} \cos \bar{\alpha}_{r}}{\sum_{r=1}^{n} \nabla_{i, r}}-\frac{\sum_{r=1}^{n}\left( \pm \frac{\Phi_{r}}{l_{r}}\right)^{3} \nabla_{i, r} \cos \bar{\alpha}_{r}\left(\cos ^{2} \bar{\alpha}_{r}+\frac{I_{\xi}^{\prime *}}{I_{\eta}^{\prime *}} \sin ^{2} \bar{\alpha}_{r}\right)}{\sum_{r=1}^{n} \nabla_{i, r}}\right\}
\end{aligned}
$$




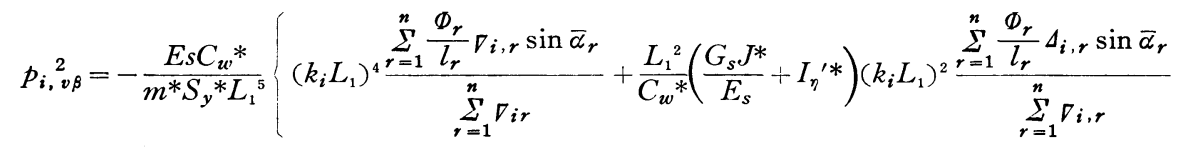

$$
\begin{aligned}
& \left.+\frac{L_{1}^{2}}{C_{w}{ }^{*}}\left(I_{\xi}{ }^{*}-I_{\eta}{ }^{*}\right) \frac{\sum_{r=1}^{n}\left(\frac{\Phi_{r}}{l_{r}}\right)^{3} \nabla_{i, r} \cos ^{2} \bar{\alpha}_{r} \sin \bar{\alpha}_{r}}{\sum_{r=1}^{n} \nabla_{i, r}}\right\} \\
& p_{i, w \beta}^{2}=\frac{E_{s} C_{w}{ }^{*}}{m^{*} S_{z}{ }^{*} L_{1}^{5}}\left\{\left(k_{i} L_{1}\right)^{4} \frac{\sum_{r=1}^{n}\left( \pm \frac{\Phi_{r}}{l_{r}}\right) \nabla_{i, r} \cos \bar{\alpha}_{r}}{\sum_{r=1}^{n} \nabla_{i, r}}+\frac{L_{1}{ }^{2}}{C_{w}{ }^{*}}\left(\frac{G_{s} J^{*}}{E_{s}}+I_{\xi}{ }^{*}\right)\left(k_{i} L_{1}\right)^{2} \frac{\sum_{r=1}^{n}\left( \pm \frac{\Phi_{r}}{l_{r}}\right) \Delta_{i, r} \cos \bar{\alpha}_{r}}{\sum_{r=1}^{n} \nabla_{i, r}}\right. \\
& +\frac{L_{1}^{2}}{C_{w}{ }^{*}}\left(I_{\eta}{ }^{*}-I_{\xi}{ }^{*}\right) \frac{\sum_{r=1}^{n}\left( \pm \frac{\Phi_{r}}{l_{r}}\right)^{3} \nabla_{i, r} \sin ^{2} \bar{\alpha}_{r} \cos \bar{\alpha}_{r}}{\sum_{r=1}^{n} \nabla_{i, r}} \\
& p_{i, \beta \beta}^{2}=\frac{E_{s} C_{w}{ }^{*}}{m^{*} I_{s}{ }^{*} L_{1}^{4}}\left\{\left(k_{i} L_{1}\right)^{4}+\frac{G_{s} J^{*} L_{1}^{2}}{E_{s} C_{w}{ }^{2}}\left(k_{i} L_{1}\right)^{2} \frac{\sum_{r=1}^{n} \Delta_{i, r}}{\sum_{r=1}^{n} \nabla_{i, r}}+\frac{L_{1}^{2}}{C_{w}{ }^{2}} \cdot \frac{\sum_{r=1}^{n}\left(\frac{\Phi_{r}}{l_{r}}\right)^{2} \nabla_{i, r}\left(I_{\xi}{ }^{*} \cos ^{2} \bar{\alpha}_{r}+I_{\eta}{ }^{*} \sin ^{2} \bar{\alpha}_{r}\right)}{\sum_{r=1}^{n} \nabla_{i, r}}\right\}
\end{aligned}
$$

where quantities $\nabla_{i, r}$ and $\Delta_{i, r}$ are given for each case.

a) Simply supported girder bridge.

$$
\nabla_{i, r}=\Delta_{i, r}=\frac{1}{2}
$$

b) Continuous girder bridge.

$$
\left.\begin{array}{l}
\nabla_{i, r}=l_{r}\left\{\left(\lambda_{i, r}{ }^{2}+\lambda_{i, r+1}{ }^{2}\right) \mu_{i, r}+\lambda_{i, r} \lambda_{i, r+1} \kappa_{i, r}\right\} \\
\Delta_{i, r}=l_{r}\left\{\left(\lambda_{i, r^{2}}+\lambda_{i, r+1}{ }^{2}\right) \nu_{i, r}+\lambda_{i, r} \lambda_{i, r+1} \theta_{i, r}\right\}
\end{array}\right\}
$$

New four parameters are also the function of $l_{r} k_{i} L_{1}$. (see Appendix)

$$
\begin{aligned}
& \mu_{i, r}=\frac{1}{2}\left(l_{r} k_{i} L_{1} \psi_{i, r} \varphi_{i, r}-\chi_{i, r}\right) \\
& \kappa_{i, r}=\zeta_{i, r}-\psi_{i, r} \\
& {\nu_{i, r}}=\frac{1}{2}\left\{\operatorname{cosec}^{2} l_{r} k_{i} L_{1}+\operatorname{cosech}^{2} l_{r} k_{i} L_{1}-\frac{1}{l_{r} k_{i} L_{1}}\left(\operatorname{coth} l_{r} k_{i} L_{1}+\cot l_{r} k_{i} L_{1}\right)\right\} \\
& \theta_{i, r}=\frac{\varphi_{i, r}}{l_{r} k_{i} L_{1}}-\left(\operatorname{cosech} l_{r} k_{i} L_{1} \operatorname{coth} l_{r} k_{i} L_{1}+\operatorname{cosec} l_{r} k_{i} L_{1} \cot l_{r} k_{i} L_{1}\right)
\end{aligned}
$$

\section{Practical Formulae}

\section{(1) Coupled vibrations of three displacements $v, w$ and $\beta$.}

a) Curved girder bridge having usual cross-section.

For a usual curved girder bridge having unsymmetrical cross-section the frequency equation is reduced to

$$
\begin{array}{ccc}
\left(p_{i, v v}^{2}-p_{i}{ }^{2}\right) A_{y} * & -\left(p_{i, w v}^{2}-p_{i}{ }^{2}\right) A_{y z}{ }^{*} & -\left(p_{i, \beta v}^{2}-p_{i}{ }^{2}\right) S_{y}{ }^{*} \\
-\left(p_{i, v w}^{2}-p_{i}{ }^{2}\right) A_{y z}{ }^{*} & \left(p_{i, w w}^{2}-p_{i}{ }^{2}\right) A_{z}{ }^{*} & \left(p_{i, \beta w}^{2}-p_{i}{ }^{2}\right) S_{z}{ }^{*} \\
-\left(p_{i, v \beta}^{2}-p_{i}{ }^{2}\right) S_{y}{ }^{*} & \left(p_{i, w \beta}^{2}-p_{i}{ }^{2}\right) S_{z}{ }^{*} & \left(p_{i, \beta \beta}^{2}-p_{i}{ }^{2}\right) I_{s}
\end{array} \mid=0
$$

It has already been mentioned that both bending vibrations in two perpendicular directions are always coupled with torsional vibration.

b) Practical formula for usual curved girder bridge.

It takes much time to find the roots $p_{i}$ of Eq. (97), but in a usual curved girder bridge the angle of inclination $\bar{\alpha}_{r}$ of the principal axes is generally small. Thus,

$$
\sin \bar{\alpha}_{r} \cong 0, \cos \bar{\alpha}_{r} \cong 1
$$

Besides, the coordinates $\bar{y}_{G, r}$ and $\bar{z}_{G, r}$ of the center of gravity $G_{r}$ are so small as compared with the radius of curvature $R_{s, r}$ that from Eq. (72) and (73) the following relations are maintained,

$$
\left.\begin{array}{l}
A_{y}{ }^{*}=A_{z}{ }^{*}=A_{s}{ }^{*} \\
A_{y z}{ }^{*}=A_{y z}{ }^{*}=0 \\
S_{y}{ }^{*}=S_{y}{ }^{*}
\end{array}\right\}
$$

Furthermore, by taking Eq. (98) into consideration

$$
p_{i, w v}^{2}=p_{i, \beta v}^{2}=p_{i, v w}^{2}=p_{i, v \beta}^{2}=0
$$




$$
\begin{aligned}
& p_{i, v v}^{2}=\frac{E_{s} I_{\eta}{ }^{*}}{m^{*} A_{s}{ }^{*} L_{1}^{4}}\left\{\left(k_{i} L_{1}\right)^{4}-\left(k_{i} L_{1}\right)^{2} \frac{\sum_{r=1}^{n}\left(\frac{\Phi_{r}}{l_{r}}\right)^{2} \Delta_{i, r}}{\sum_{r=1}^{n} \nabla_{i, r}}\right\} \\
& p_{i, w w}^{2}=\frac{E_{s} I_{\xi}{ }^{*}}{m^{*} A_{s}{ }^{*} L_{1}{ }^{4}}\left\{\left(k_{i} L_{1}\right)^{4}-\left(k_{i} L_{1}\right)^{2} \frac{\sum_{r=1}^{n}\left(\frac{\Phi_{r}}{l_{r}}\right)^{2} \Delta_{i, r}}{\sum_{r=1}^{n} \nabla_{i, r}}\right\} \\
& \left.p_{i, \beta m}^{2}=\frac{E_{s} I_{\xi}^{\prime *}}{m^{*} S_{z^{\prime}}{ }^{\prime} L_{1}^{3}} \mid\left(k_{i} L_{1}\right)^{2} \frac{\sum_{r=1}^{n}\left( \pm \frac{\Phi_{r}}{l_{r}}\right) \Delta_{i, r}}{\sum_{r=1}^{n} \nabla_{i, r}}-\frac{\sum_{r=1}^{n}\left( \pm \frac{\Phi_{r}}{l_{r}}\right)^{3} \nabla_{i, r}}{\sum_{r=1}^{n} \nabla_{i, r}}\right\} \\
& p_{i, w \beta}^{2}=\frac{E_{s} C_{w^{*}}{ }^{*} S_{z}^{*} L_{1}^{5}}{m^{5}}\left\{\left(k_{i} L_{1}\right)^{4} \frac{\sum_{r=1}^{n}\left( \pm \frac{\Phi_{r}}{l_{r}}\right) \nabla_{i, r}}{\sum_{r=1}^{n} \nabla_{i, r}}+\frac{L_{1}^{2}}{C_{w}{ }^{2}}\left(\frac{G_{s} J^{*}}{E_{s}}+I_{\xi}^{* *}\right)\left(k_{i} L_{1}\right)^{2} \frac{\sum_{r=1}^{n}\left( \pm \frac{\Phi_{r}}{l_{r}}\right) \Delta_{i, r}}{\sum_{r=1}^{n} \nabla_{i, r}}\right\} \\
& p_{i, \beta \beta}^{2}=\frac{E_{s} C_{w^{*}}{ }^{2}}{m^{*} I_{s} * L_{1}{ }^{4}}\left\{\left(k_{i} L_{1}\right)^{4}+\frac{G_{s} J * L_{1}{ }^{2}}{E_{s} C_{w}{ }^{*}}\left(k_{i} L_{1}\right)^{2} \frac{\sum_{r=1}^{n} \Delta_{i, r}}{\sum_{r=1}^{n} \nabla_{i, r}}+\frac{I_{\xi}{ }^{\prime} * L_{1}{ }^{2}}{C_{w}{ }^{*}} \cdot \frac{\sum_{r=1}^{n}\left(\frac{\Phi_{r}}{l_{r}}\right)^{2} \nabla_{i, r}}{\sum_{r=1}^{n} \nabla_{i, r}}\right\}
\end{aligned}
$$

The frequency equation can be rewritten in the following form.

$$
\left|\begin{array}{ccc}
\left(p_{i, v v}{ }^{2}-p_{i}{ }^{2}\right) A_{s}{ }^{*} & 0 & p_{i}{ }^{2} S_{y}{ }^{*} \\
0 & \left(p_{i, w w}{ }^{2}-p_{i}{ }^{2}\right) A_{s}{ }^{*} & \left(p_{i, \beta w}{ }^{2}-p_{i}{ }^{2}\right) S_{z}{ }^{*} \\
p_{i}{ }^{2} S_{y}{ }^{*} & \left(p_{i, w \beta}{ }^{2}-p_{i}{ }^{2}\right) S_{z}{ }^{*} & \left(p_{i, \beta \beta}{ }^{2}-p_{i}{ }^{2}\right) I_{s} *
\end{array}\right|=0
$$

c) Straight girder bridge having an unsymmetri- and in Eq. (101)

cal cross-section.

If a straight girder bridge has an unsymmetrical cross-section, the following relations can be available besides Eq. (99)

$$
\Phi_{r}=0(r=1,2,3, \cdots, n)
$$

Thereby,

$$
p_{i, \beta \omega}^{2}=p_{i, \omega \beta}^{2}=0
$$

$$
\begin{aligned}
& S_{z} *=S_{z}{ }^{*} \text { * } \\
& \left|\begin{array}{ccc}
\left(p_{i}{ }^{2}{ }_{v v}-p_{i}{ }^{2}\right) A_{s}{ }^{*} & 0 & p_{i}{ }^{2} S_{y}{ }^{*} \\
0 & \left(p_{i w w}{ }^{2}-p_{i}{ }^{2}\right) A_{s}{ }^{*} & -p_{i}{ }^{2} S_{z}{ }^{*} \\
p_{i}{ }^{2} S_{y}{ }^{*} & -p_{i}{ }^{2} S_{z}{ }^{*} & \left(p_{i, \beta \beta}{ }^{2}-p_{i}{ }^{2}\right) I_{s}{ }^{*}
\end{array}\right|=0
\end{aligned}
$$

For this case, from the frequency equation (97),

Now, all the frequency equations (97), (102) and (106) become a cubic equation;

$$
a\left(p_{i}{ }^{2}\right)^{3}-b_{i}\left(p_{i}{ }^{2}\right)^{2}+c_{i} p_{i}{ }^{2}-d_{i}=0
$$

Especially, in Eq. (102) the coefficients $a, b_{i}, c_{i}$, and $d_{i}$ have following values,

$$
\begin{aligned}
& a=A_{s} * I_{S} *-S_{y}{ }^{* 2}-S_{z} * S_{z}{ }^{*} * \\
& b_{i}=A_{s}{ }^{*} I_{S} *\left(p_{i, v v}^{2}+p_{i, w w}^{2}+p_{i, \beta \beta}^{2}\right)-S_{y}{ }^{2} p_{i, w w}^{2}-S_{z} * S_{z}{ }^{2} *\left(p_{i, v v}^{2}+p_{i, w \beta}^{2}+p_{i, \beta w}^{2}\right) \\
& c_{i}=A_{s} * I_{S} *\left\{p_{i, v v}^{2}\left(p_{i, w w}^{2}+p_{i, \beta \beta}^{2}\right)+p_{i, \omega \omega}^{2} p_{i, \beta \beta}^{2}\right\}-S_{z} * S_{z}{ }^{2} *\left\{p_{i, v v}^{2}\left(p_{i, w \beta}^{2}+p_{i, \beta w}^{2}\right)+p_{i, w \beta}^{2} p_{i, \beta \omega}^{2}\right\} \\
& d_{i}=\left(A_{s} * I_{S}{ }^{*} p_{i, w w}^{2} p_{i, \beta \beta}^{2}-S_{z} * S_{z} * p_{i, w \beta}^{2} p_{i, \beta w}^{2}\right) p_{i, v v}^{2}
\end{aligned}
$$

but it will be sufficient for a straight girder bridge to use Eq. (103), (104) and (105) in Eq. (108).

For the triple coupled vibrations, the complete solution of natural frequencies $p_{i}$ will be obtained by solving the cubic equation (107). The approximate roots of this equation can easily find by means of Cardano's or Newton's method. All these roots $p_{i}$ are real for each mode $(i=1,2,3, \cdots)$, and $p_{i, \mathrm{I}}$ $<p_{i, \text { II }}<p_{i \text {, II }}$ represents three circular frequencies in these coupled vibrations.

It should be understood that the inequality $p_{i, \mathrm{I}}<$ $p_{i, w w}, p_{i, w w}<p_{i, \mathbb{I}}<p_{i, \beta \beta}$, and $p_{i, \mathbb{I}}>p_{i, \beta \beta}$ will be established among three roots $p_{i, \mathrm{I}}, p_{i, \mathrm{I}}, p_{i, \mathrm{II}}$ and uncoupled frequencies $p_{i, v v}, p_{i, w w}, p_{i, \beta s}$, if an inequarity $p_{i, w w}<p_{i, v v}<p_{i, \beta \beta}$ is assumed.

\section{(2) Coupled vibration of two displacements $\boldsymbol{w}$ and $\beta$.}

a) Curved girder bridge having special cross section $\left(S_{y} *=S_{y}{ }^{*}=0\right)$.

If there is eccentricity of the center of gravity only in the horizontal direction and the angle of inclination of the principal axes disappears, that is, the statical moment about the axis $S y$ will be equal to zero, 
$S_{y}{ }^{*}=S_{y}{ }^{*}=0$

Therefore, the following frequency equation will be obtained,

$$
\left|\begin{array}{ccc}
\left(p_{i, v v}^{2}-p_{i}{ }^{2}\right) A_{s} * & 0 & 0 \\
0 & \left(p_{i, w w}^{2}-p_{i}{ }^{2}\right) A_{s} * & \left(p_{i, \beta w}^{2}-p_{i}{ }^{2}\right) S_{z}{ }^{*} \\
0 & \left(p_{i, \omega \beta}^{2}-p_{i}{ }^{2}\right) S_{z} * & \left(p_{i, \beta \beta}^{2}-p_{i}{ }^{2}\right) I_{S}
\end{array}\right|=0
$$

From the above equation, it is clear that the bending vibration in the vertical direction is coupled with torsional one.

b) Straight girder bridge having symmetrical cross-section about one axis $\left(S_{y}{ }^{*}=0\right)$.

If a straight girder bridge has a symmetrical cross-section about the vertical axis,we may adopt

$$
\begin{aligned}
& a=A_{s} * I_{S} *-S_{z} * S_{z}{ }^{*} \\
& b_{i}=A_{s} * I_{S} *\left(p_{i, w w}^{2}+p_{i, \beta \beta}^{2}\right)-S_{z} * S_{z}{ }^{\prime *}\left(p_{i, \beta w}^{2}+p_{i, w \beta}^{2}\right) \\
& c_{i}=A_{s} * I_{S} * p_{i, w w}^{2} p_{i, \beta \beta}^{2}-S_{z} * S_{z}{ }^{\prime} p_{i, \beta w}^{2} p_{i, \omega \beta}^{2}
\end{aligned}
$$

Consequently, there exist two coupled frequencies $p_{i, \mathrm{I}}$ and $p_{i, \text { II }}$ for the $i$ th mode. If we assume, for instance, $p_{i, w w}<p_{i, \beta \beta}$, the relations between $p_{i, w w}$ (or $p_{i, \beta \beta}$ ) and the root $p_{i, \mathrm{I}}$ (or $p_{i, \mathrm{II}}$ ) will be given as $p_{i, \mathrm{I}}<p_{i, w w}$ and $p_{i, \mathrm{II}}>p_{i, \beta \beta}$.

\section{(3) Coupled vibrations of two displacements $\boldsymbol{v}$ and $\boldsymbol{\beta}$.}

a) Special curved girder bridge $\left(S_{z}{ }^{*}=S_{z}{ }^{*}=0\right.$, $\alpha=\pi / 2)$.

In a special curved girder bridge where the crosssection is bisymmetric and has a curvature only in the vertical plane, that is to say, when a straight
Eq. (104) and (105) in Eq. (110).

The solutions of Eq. (110) will have the following values,

$$
\left.\begin{array}{l}
\left.p_{i}, \quad \mathrm{I} \quad \text { or } \quad \mathrm{II}=\left|\sqrt{\frac{b_{i \pm \sqrt{b_{i}{ }^{2}-4 a c_{i}}}}{2 a}}\right|\right\} . \\
p_{i}, \quad \text { III }=\left|p_{i, v v}\right|
\end{array}\right\}
$$

where the coefficients $a, b_{i}$, and $c_{i}$ are as follows;

girder bridge has a circular camber with comparatively small curvature, we can put,

$$
\begin{aligned}
\sin \bar{\alpha}_{r} & =1, \cos \bar{\alpha}_{r}=0 \cdots \\
(r & =1,2,3, \cdots, n)
\end{aligned}
$$

Furthermore, if the eccentric distance $\bar{y}_{G, r}$ is small as compared with the radius of curvature $R_{S, r}$, from Eq. (72) and (73) we obtain,

$$
\begin{aligned}
& A_{y}{ }^{*}=A_{z}{ }^{*}=A_{s}{ }^{*} \\
& A_{y z}{ }^{*}=A_{y z}{ }^{*}=0 \\
& S_{z}{ }^{*}=S_{z}{ }^{*}=0
\end{aligned}
$$

Accordingly, the values $p_{i, v v}^{2}, p_{i, w w}^{2}, p_{i, \beta \beta}^{2}$, .....etc. will be computed by

$$
\begin{aligned}
& p_{i, v v}^{2}=\frac{E_{s} I_{\eta}{ }^{*}}{m^{*} A_{s}{ }^{*} L_{1}{ }^{4}}\left\{\left(k_{i} L_{1}\right)^{4}-\left(k_{i} L_{1}\right)^{2} \frac{\sum_{r=1_{1}}^{n}\left(\frac{\Phi_{r}}{l_{r}}\right)^{2} \Delta_{i, r}}{\sum_{r=1}^{n} \nabla_{i, r}}\right\} \\
& p_{i, \beta v}^{2}=-\frac{E_{s} I_{\eta}{ }^{*}}{m^{*} S_{y^{\prime}}{ }^{*} L_{1}{ }^{3}}\left\{\left(k_{i} L_{1}\right)^{2} \frac{\sum_{r=1}^{n} \frac{\Phi_{r}}{l_{r}} \Delta_{i, r}}{\sum_{r=1}^{n} \nabla_{i, r}}-\frac{\sum_{r=1}^{n}\left(\frac{\Phi_{r}}{l_{r}}\right)^{3} \nabla_{i, r}}{\sum_{r=1}^{n} \nabla_{i, r}}\right\} \\
& p_{i, w w}^{2}=\frac{E_{s} I_{\xi}{ }^{*}}{m^{*} A_{s}^{*} L_{1}^{4}}\left\{\left(k_{i} L_{1}\right)^{4}-\left(k_{i} L_{1}\right)^{2} \frac{\sum_{r=1}^{n}\left(\frac{\Phi_{r}}{l_{r}}\right)^{2} \Delta_{i, r}}{\sum_{r=1}^{n} \nabla_{i, r}}\right\} \\
& p_{i, v \beta}^{2}=-\frac{E_{s} C_{w}{ }^{*}}{m^{*} S_{y}{ }^{*} L_{1}^{5}}\left\{\left(k_{i} L_{1}\right)^{4} \frac{\sum_{r=1}^{n} \frac{\Phi_{r}}{l_{r}} \nabla_{i, r}}{\sum_{r=1}^{n} \nabla_{i, r}}-\frac{L_{1}^{2}}{C_{w}{ }^{*}}\left(\frac{G_{s} J^{*}}{E_{s}}+I_{\eta}{ }^{*}\right)\left(k_{i} L_{1}\right)^{2} \frac{\sum_{r=1}^{n} \frac{\Phi_{r}}{l_{r}} \Delta_{i, r}}{\sum_{r=1}^{n} \nabla_{i, r}}\right\} \\
& p_{i, \beta \beta}^{2}=\frac{E_{s} C_{w}{ }^{*}}{m^{*} I_{s}{ }^{*} L_{1}{ }^{4}}\left\{\left(k_{i} L_{1}\right)^{4}+\frac{G_{s} J^{*} L_{1}{ }^{2}}{E_{s} C_{w}{ }^{*}}\left(k_{i} L_{1}\right)^{2} \frac{\sum_{r=1}^{n} \Delta_{i, r}}{\sum_{r=1}^{n} \nabla_{i, r}}+\frac{I_{\eta}{ }^{*} * L_{1}{ }^{2}}{C_{w}{ }^{2}} \cdot \frac{\sum_{r=1}^{n}\left(\frac{\Phi_{r}}{l_{r}}\right)^{2} \nabla_{i, r}}{\sum_{r=1}^{n} \nabla_{i, r}}\right\}
\end{aligned}
$$

The frequency equation will become as follows;

$$
\left|\begin{array}{ccc}
\left(p_{i, v v}^{2}-p_{i}{ }^{2}\right) A_{s} * & 0 & -\left(p_{i, \beta v}^{2}-p_{i}{ }^{2}\right) S_{y}{ }^{*} \\
0 & \left(p_{i, w w}^{2}-p_{i}{ }^{2}\right) A_{s} * & 0 \\
-\left(p_{i, v \beta}^{2}-p_{i}{ }^{2}\right) S_{y} * & 0 & \left(p_{i, \beta \beta}^{2}-p_{i}{ }^{2}\right) I_{s}^{*}
\end{array}\right|=0
$$

It can be seen from this equation that a bending vibration in the vertical direction is independent, 
while a bending vibration in the horizontal direction coupled with the torsional one.

b) Practical formula for S-shaped curved girder bridge.

Let us now consider another special curved girder bridge as shown in Fig.10. There, Fig.10(a) shows a two equal span $\mathrm{S}$-shaped continuous curved girder bridge, while $10($ b) a three span $S$-shaped continuous bridge where two side spans have an equal length but reverse curvature. In the center span, the girder is straight and has a bisymmetrical cross-section.

Devoting our attention to the signs of $R_{S, r}$ and $\Phi_{r}$, we understand by observing Eq. (72) and (73) that the neccessary equation will coincide with Eq. (114), then the equation to give the values $p_{i, v v}^{2}$, $p_{i, w w}^{2}, p_{i, \beta \beta}^{2}, \cdots$ etc. will be identical with Eq. (101), where Eq. (105) is also available.

Thus, the frequency equation will be obtained as follows.

$$
\left|\begin{array}{ccc}
\left(p_{i, v v}{ }^{2}-p_{i}{ }^{2}\right) A_{s}{ }^{*} & 0 & -p_{i}{ }^{2} S_{y}{ }^{*} \\
0 & \left(p_{i, w w}{ }^{2}-p_{i}{ }^{2}\right) A_{s}{ }^{*} & 0 \\
p_{i}{ }^{2} S_{y}{ }^{*} & 0 & \left(p_{i, \beta \beta}{ }^{2}-p_{i}{ }^{2}\right) I_{S} *
\end{array}\right|=0
$$

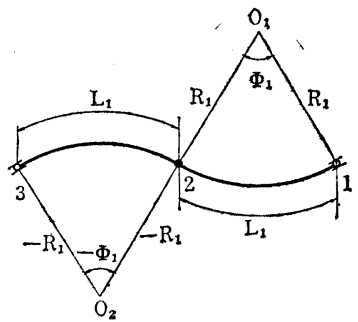

(a) two-span

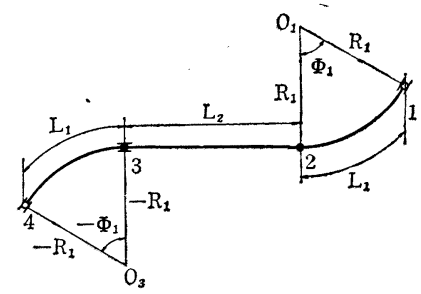

(b) three-span

Fig. 10 Plane shape of S-shaped curved girder bridge.
This equation shows that the bending vibration in the vertical direction is independent and that the bending vibration in the horizontal direction is coupled with the torsional one.

c) Practical formula for a straight girder bridge.

The cross-section of a usual straight girder bridge is bisymmetrical and that has no curvature about any axis, so using Eq. (104) in Eq. (115) we can put

$$
S_{y}{ }^{*}=S_{y}{ }^{*}
$$

and

$$
p_{i, \beta v}^{2}=p_{i, v \beta}^{2}=0
$$

$$
\begin{aligned}
& a=A_{s} * I_{S} *-S_{y} * S_{y}{ }^{\prime *} \\
& b_{i}=A_{s} * I_{S} *\left(p_{i, v v}^{2}+p_{i, \beta \beta}^{2}\right)-S_{y} * S_{y}{ }^{2} *\left(p_{i, \beta v}^{2}+p_{i, v \beta}^{2}\right) \\
& c_{i}=A_{s} * I_{S} * p_{i, v v}^{2} p_{i, \beta \beta}^{2}-S_{y} * S_{y}{ }^{\prime}{ }^{2} p_{i, \beta v}^{2} p_{i, v \beta}^{2}
\end{aligned}
$$

From this, two coupled frequencies $p_{i, \text { II }}<p_{i \text {, II }}$ can be determined and if the value $p_{i, \beta \beta}$ is greater than $p_{i, v v}$, we find the inequality $p_{i, \mathrm{I}}<p_{i, v v}, p_{i, \mathrm{II}}>p_{i, \beta \beta}$.

(4) Uncoupled vibration.

$$
\left|\begin{array}{ccc}
\left(p_{i, v v}{ }^{2}-p_{i}{ }^{2}\right) A_{s}{ }^{*} & 0 & 0 \\
0 & \left(p_{i, w w}^{2}-p_{i}{ }^{2}\right) A_{s} * & 0 \\
0 & 0 & \left(p_{i, \beta \beta}^{2}-p_{i}{ }^{2}\right) I_{S}
\end{array}\right|=0
$$

If a straight girder bridge has two axes of symmetry, namely,

$$
S_{y}{ }^{*}=S_{z}{ }^{*}=0
$$

The frequency equation will become,

obtained by

This indicates clearly that both bending vibration in two perpendicular directions and the torsional vibration are always uncoupled and independent each other. So, the circular frequencies can be

$$
\left.\begin{array}{l}
p_{i}=\left|p_{i, v v}\right| \\
p_{i}=\left|p_{i, w w}\right| \\
p_{i}=\left|p_{i, \beta \beta}\right|
\end{array}\right\}
$$




\section{EXPERIMENTAL STUDY.}

In order to verify the above theory, both field and model tests were conducted about four types of curved girder bridges constructed near the city of Osaka.
1. Example of Triple Coupled Vibrations.

a) Nishinomiya Bridge at the interchange of Meishin Express-Way.

This bridge is fabricated as a two equal span continuous curved girder bridge as shown in Fig. 11 (a). The bridge consists of two box girders and

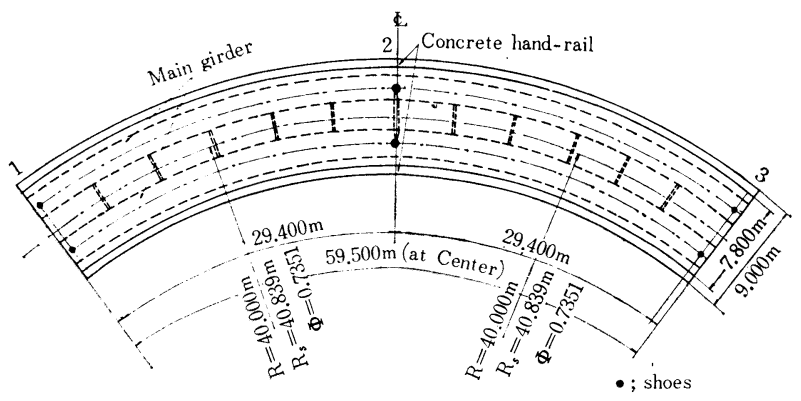

(a) general plane

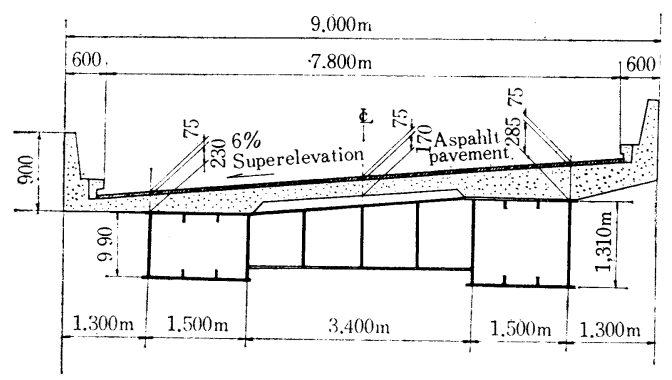

(b) typical cross-section

Fig. 11 Detail of Nishinomiya bridge.

a reinforced concrete slab as shown in Fig. 11 (b).

Although the bridge was calculated as a noncomposite girder in the design, it was recognized from the statical loading tests the main box girders resisted as a composite girder to one vehicle, be- cause there were considerable composite actions by means of the slab clamps and the frictional resistances between the top cover plates of the box girders and a concrete slab.

Table 1 Cross sectional quantities of test bridges.

\begin{tabular}{l|c|c|c|c}
\hline \multicolumn{1}{c|}{ Values } & $\begin{array}{c}\text { Nishinomiya } \\
\text { Br. }\end{array}$ & Nagara Br. & Jusoo Br. & $\begin{array}{c}\text { Nakanoshima } \\
\text { Br. (Model) }\end{array}$ \\
\hline$A_{S^{*}\left(\mathrm{~cm}^{2}\right)}$ & $1.2713 \times 10^{4}$ & $6.3299 \times 10^{3}$ & $9.674 \times 10^{3}$ & $5.9501 \times 10^{1}$ \\
$S_{y}{ }^{*}\left(\mathrm{~cm}^{3}\right)$ & $7.3040 \times 10^{5}$ & $-8.9204 \times 10^{4}$ & - & $1.7999 \times 10^{2}$ \\
$S_{z^{*}}\left(\mathrm{~cm}^{3}\right)$ & $5.3531 \times 10^{5}$ & $1.4071 \times 10^{5}$ & $4.452 \times 10^{5}$ & - \\
$S_{z^{\prime}}{ }^{*}\left(\mathrm{~cm}^{3}\right)$ & $4.3197 \times 10^{5}$ & $1.1440 \times 10^{5}$ & $4.013 \times 10^{5}$ & - \\
$I_{\xi}^{\prime *}\left(\mathrm{~cm}^{4}\right)$ & $1.3834 \times 10^{7}$ & $1.5431 \times 10^{7}$ & $1.057 \times 10^{7}$ & $6.0240 \times 10^{2}$ \\
$I_{r_{t}^{\prime}}{ }^{*}\left(\mathrm{~cm}^{4}\right)$ & $3.4881 \times 10^{8}$ & $9.5503 \times 10^{7}$ & $1.765 \times 10^{8}$ & $1.7665 \times 10^{4}$ \\
$I_{S^{*}}\left(\mathrm{~cm}^{4}\right)$ & $4.2203 \times 10^{8}$ & $1.1467 \times 10^{8}$ & $2.086 \times 10^{8}$ & $1.9204 \times 10^{4}$ \\
$J^{*}\left(\mathrm{~cm}^{4}\right)$ & $9.9898 \times 10^{6}$ & $2.7333 \times 10^{7}$ & $2.173 \times 10^{5}$ & $7.5834 \times 10^{2}$ \\
$C_{w^{*}}\left(\mathrm{~cm}^{6}\right)$ & $8.2144 \times 10^{11}$ & $4.9423 \times 10^{10}$ & $6.170 \times 10^{11}$ & $2.2474 \times 10^{5}$ \\
\hline
\end{tabular}

Thereby, to find the natural frequencies, all the cross sectional quantities may be given as the composited values and be assumed the ratio $n$ of Young's modulus of steel $E_{s}$ to that of concrete $E_{c}$ to be equal to 7 .

Table 1 shows their mean values of these quantities. The values of shape Parameters $k_{i} L_{i}$ and the values $p_{i, v v}^{2}, p_{i, w w}^{2}, p_{i, \beta, 3}^{2}, \cdots$, etc., calculated by the practical formula (101), are shown in Table 2 . From these input data, the quantities $p_{i, \mathrm{I} \sim \text { III }}^{2}$ and the corresponding natural frequencies $f_{i, \mathrm{I} \sim \text { III }}$ have been obtained from Eq. (107) as shown in Table 3.

In this table, the numbers in the parenthesis represent the order of the modes. When the values $p_{i, \mathrm{I}}^{2} \sim$ III in Table 3 are compared with those of $p_{i, v v}^{2}$, $p_{i, w w}^{2}$ and $p_{i, \beta \beta}^{2}$ in Table 2, it is clear that in the 
Table 2 Values $k_{i} L_{1}$ and $p_{i, v v}^{2}, \underset{p_{i} w w}{2}, p_{i, \beta \beta}^{2}, \cdots$. ect, (Nishino-miya $\mathrm{Br}$.)

\begin{tabular}{c|c|c|c|c|c|c}
\hline$i$ & $k_{i} L_{1}$ & $p_{i, v v}^{2}$ & $p_{i, w w}^{2}$ & $p_{i, \beta w}^{2}$ & $p_{i, \omega \beta}^{2}$ & $p_{i, \beta \beta}^{2}$ \\
\hline 1 & 3.142 & $8.2744 \times 10^{3}$ & $3.2816 \times 10^{2}$ & $2.1589 \times 10^{3}$ & $2.4455 \times 10^{3}$ & $3.8029 \times 10^{3}$ \\
2 & 3.927 & $2.0357 \times 10^{4}$ & $8.0735 \times 10^{3}$ & $4.6528 \times 10^{3}$ & $5.2542 \times 10^{3}$ & $7.6008 \times 10^{3}$ \\
3 & 6.287 & $1.3815 \times 10^{5}$ & $5.4789 \times 10^{3}$ & $9.0107 \times 10^{3}$ & $1.1342 \times 10^{4}$ & $2.1096 \times 10^{4}$ \\
4 & 7.069 & $2.2163 \times 10^{6}$ & $8.7894 \times 10^{4}$ & $1.3341 \times 10^{4}$ & $1.6962 \times 10^{4}$ & $6.9325 \times 10^{4}$ \\
\hline
\end{tabular}

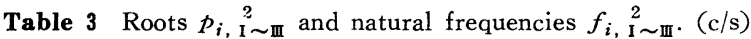
(Nishino-miya Br.)

\begin{tabular}{c|c|c|c||c|c|c}
\hline$i$ & $p_{i, \mathrm{I}}^{2}$ & $p_{i, \mathrm{II}}^{2}$ & $p_{i, \mathrm{II}}^{2}$ & $f_{i, \mathrm{I}}$ & $f_{i, \mathrm{II}}$ & $f_{i, \text { III }}$ \\
\hline 1 & $2.7831 \times 10^{2}$ & $3.5570 \times 10^{3}$ & $9.9469 \times 10^{3}$ & $2.6551(1)$ & $9.4922(3)$ & $15.873(7)$ \\
2 & $6.9468 \times 10^{2}$ & $7.2381 \times 10^{3}$ & $2.3999 \times 10^{4}$ & $4.1948(2)$ & $12.735(5)$ & 24.655 \\
3 & $5.4204 \times 10^{3}$ & $2.1043 \times 10^{4}$ & $1.5704 \times 10^{5}$ & $11.717(4)$ & 23.087 & 63.072 \\
4 & $8.7627 \times 10^{3}$ & $6.8341 \times 10^{4}$ & $2.5878 \times 10^{5}$ & $14.898(6)$ & 41.606 & 80.972 \\
\hline
\end{tabular}

1 st, $2 \mathrm{nd}, 4$ th and 6 th modes the vertical bending vibration are predominant, in the $3 \mathrm{rd}$ and 5 th the torsional vibration, and in the 7 th the horizontal bending vibration.

Table 4 shows the amplitudes $\bar{v}_{i}$ and $\bar{b}_{i}$ when $\bar{w}_{i}$ is assumed to be equal to unit. Accordingly, the patterns of the vibrations can be illustrated in Fig. 12, where the skelton of the bridge is sketched in a straight line for the sake of clearness.

The free vibration tests were conducted by driving a 20 tons load truck at the various speed. The strain and the deflection were measured after the

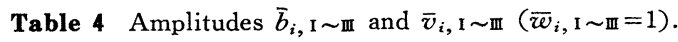

(Nishino-miya $\mathrm{Br}$.)

\begin{tabular}{|c|c|c|c|c|c|c|}
\hline$i$ & $\vec{b}_{i}, \mathbf{I}$ & $\bar{b}_{i}$, II & $\bar{b}_{i}$, III & $\bar{v}_{i}, \mathrm{I}$ & $\bar{v}_{i}$, II & $\bar{v}_{i}$, 息 \\
\hline 1 & $-7.8013 \times 10^{-4}$ & $-6.7969 \times 10^{-2}$ & $-3.6348 \times 10^{-4}$ & $9.3561 \times 10^{-2}$ & $4.9369 \times 10^{-2}$ & $-1.2453 \times 10^{-1}$ \\
\hline 2 & $-8.3772 \times 10^{-4}$ & $-7.3206 \times 10^{-2}$ & $-3.5280 \times 10^{-2}$ & $6.4571 \times 10^{-5}$ & $2.7276 \times 10^{-2}$ & $-8.6217 \times 10^{-2}$ \\
\hline 3 & $-4.7983 \times 10^{-4}$ & $-3.8069 \times 10^{-2}$ & $-3.0132 \times 10^{-2}$ & $1.5291 \times 10^{-5}$ & $2.7093 \times 10^{-3}$ & $-3.6317 \times 10^{-2}$ \\
\hline 4 & $-1.7183 \times 10^{-4}$ & $-3.1866 \times 10^{-2}$ & $-2.9976 \times 10^{-2}$ & $4.3412 \times 10^{-6}$ & $3.1222 \times 10^{-3}$ & $-2.3581 \times 10^{-2}$ \\
\hline
\end{tabular}

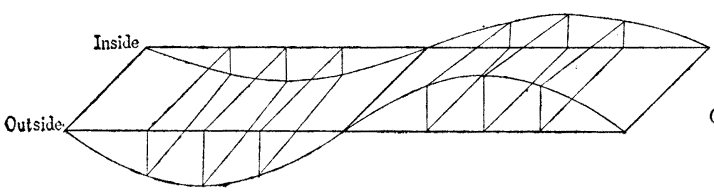

(a) 1 st mode

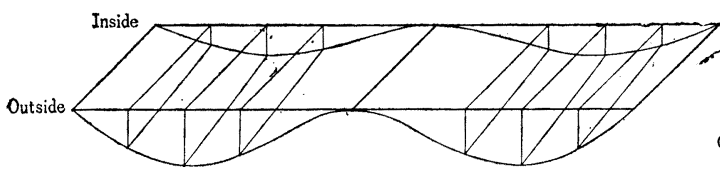

(b) 2 nd mode

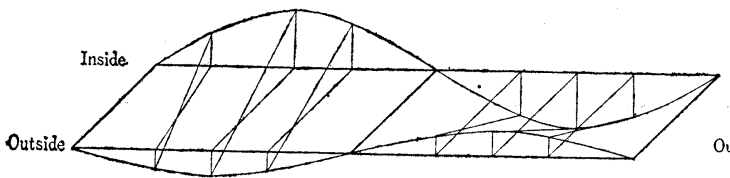

(c) 3 rd mode

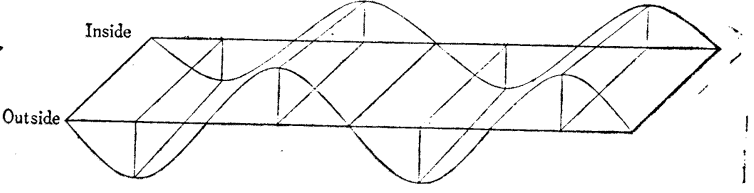

(d) 4 th mode

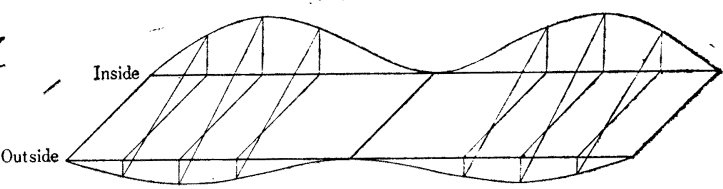

(e) 5 th mode

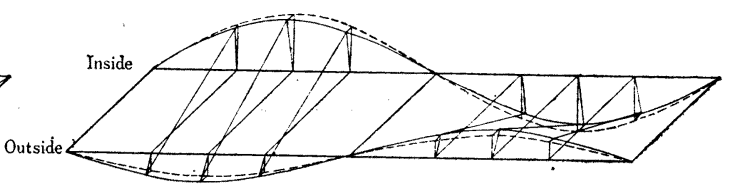

(g) 7 th mode

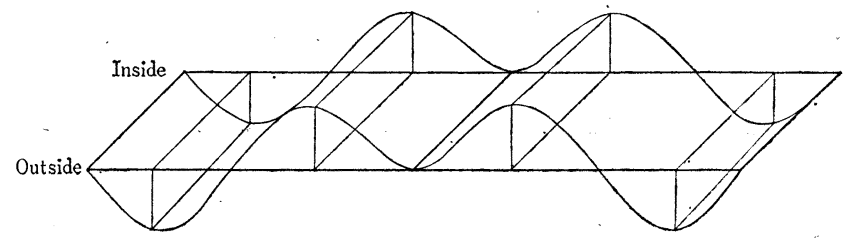

(f) 6 th mode

Fig. 12 Patterns of vibration. 


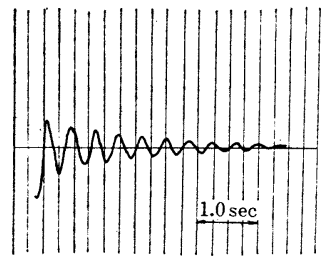

(a) deflection

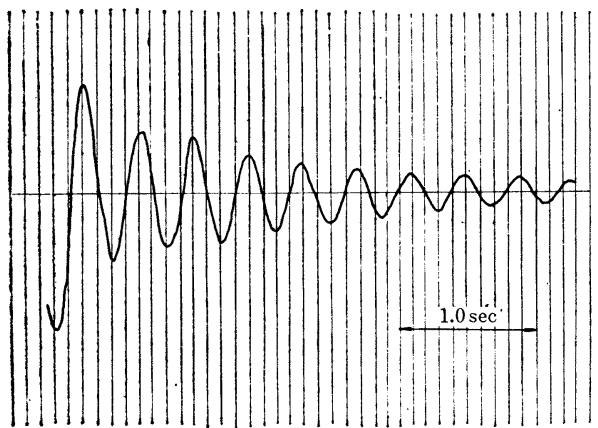

(b) stress

Fig. 13 Free vibration records (Nishinomiya bridge)

truck had passed through the end support. Fig. 13 illustrates the typical data recorded by an osillograph, in which the first mode of vibration was observed while the other higher frequencies could not appear appreciably.

The mean experimental value of the lowest natural frequency $\left(f_{1, \mathrm{I}}\right)_{\text {test }}$ was 2.60 cycle/sec.. If we compare this frequency with theoretical one $\left(f_{1}, \mathrm{I}\right)$ cal $=2.655$ cycle/sec., the ratio between $\left(f_{1}, \mathrm{I}\right)_{\text {cal }}$ and $\left(f_{1}, \mathrm{I}\right)$ test is equal to $102 \%$.

By the way, if we calculate the frequencies as an. uncoupled vibration or as a straight girder bridge, $\left(f_{1}\right)_{\text {uncouple }}=p_{i, w w} / 2 \pi=2.883 \mathrm{c} / \mathrm{s}$ or $\left(f_{1}\right)_{\text {straight }}=$ $2.965 \mathrm{c} / \mathrm{s}$ will be obtained for the lowest mode, so that the ratio, $\left(f_{1}\right)_{\text {uncouple }} /\left(f_{1, \mathrm{I}}\right)_{\text {test }}$ and $\left(f_{1}\right)_{\text {straight }} /$ $\left(f_{1, \mathrm{I}}\right)_{\text {test }}$, is $111 \%$ and $114 \%$ respectively. It is about $22 \sim 28 \%$ over-estimating the rigidities of this bridge.

Consequently, it is impossible to explain the free vibration of a curved girder bridge of this type without taking into the consideration the influence of curvature and coupled vibrations.

\section{b) Nagara Bypass Bridge in Osaka City.}

This bridge is a three span continuous curved girder bridge having one main box girder and the steel deck as shown in Fig. 14. The span length and radii of curvature are different from each other. At one of the middle supports, the shoes are not: situated in the radial direction of the main girder. However, it is assumed, for the sake of simplicity, the shoes are attached in the radial direction as shown with a dotted line in Fig. 14.

(a) general plane

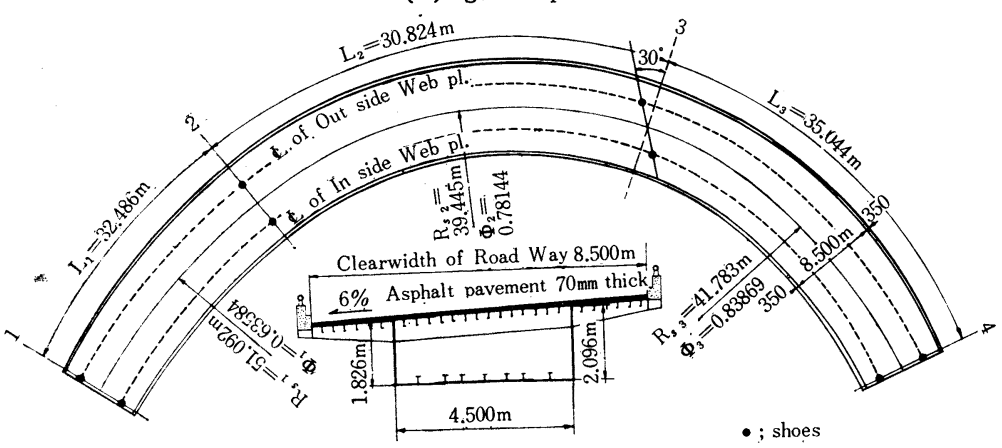

(b) typical cross-section

Fig. 14 Detail of Nagara bridge.

Table 5 Values $k_{i} L_{1}$ and $p_{i, v v}{ }^{2}, p_{i, w w}{ }^{2}, p_{i, \beta \beta^{2}}{ }^{2}, \cdots$ etc.

(Nagara Br.)

\begin{tabular}{|c|c|c|c|c|c|c|}
\hline$i$ & $k_{i} L_{t}$ & $p_{i, v v^{2}}$ & $p_{i, w w^{2}}$ & $p_{i, \beta w^{2}}$ & $p_{i, w \beta^{2}}$ & $p_{i, \beta \hat{\beta}}^{2}$ \\
\hline 1 & 3.0774 & $3.0535 \times 10^{3}$ & $4.9337 \times 10^{2}$ & $6.3640 \times 10^{3}$ & $9.1097 \times 10^{3}$ & $2.4272 \times 10^{4}$ \\
\hline 2 & 3.4700 & $5.1154 \times 10^{3}$ & $8.2654 \times 10^{2}$ & $2.0895 \times 10^{3}$ & $3.0393 \times 10^{3}$ & $2.4147 \times 10^{4}$ \\
\hline 3 & 4.3829 & $1.2908 \times 10^{4}$ & $2.0857 \times 10^{3}$ & $9.0236 \times 10^{3}$ & $1.3082 \times 10^{4}$ & $3.3190 \times 10^{4}$ \\
\hline 4 & 6.0936 & $4.9129 \times 10^{4}$ & $7.9381 \times 10^{3}$ & $3.3888 \times 10^{3}$ & $4.7437 \times 10^{4}$ & $9.2430 \times 10^{4}$ \\
\hline
\end{tabular}

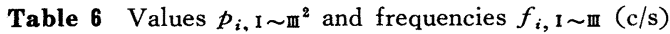

(Nagara Br.)

\begin{tabular}{|c|c|c|c|c|c|c|}
\hline$i$ & $p_{i, I^{2}}$ & $p_{i}, \mathrm{II}^{2}$ & $p_{i}, \mathrm{II}^{2}$ & $f_{i, \mathrm{I}}$ & $f_{i, \text { II }}$ & $f_{i}$, III \\
\hline 1 & $4.4564 \times 10^{2}$ & $3.0487 \times 10^{3}$ & $2.4847 \times 10^{4}$ & 3.3598 (1) & $8.7881(4)$ & 25.088 \\
\hline 2 & $8.2387 \times 10^{2}$ & $5.1005 \times 10^{3}$ & $2.4952 \times 10^{4}$ & $4.5683(2)$ & 11.3660 (5) & 25.148 \\
\hline 3 & $2.0307 \times 10^{3}$ & $1.2820 \times 10^{1}$ & $3.1458 \times 10^{4}$ & 7.1721 & 18.0200 & 29.415 \\
\hline 4 & $7.9343 \times 10^{3}$ & $4.8554 \times 10^{4}$ & $9.6729 \times 10^{4}$ & $14.1770(6)$ & 35.0690 & 49.500 \\
\hline
\end{tabular}


Table 1 shows the cross sectional quantities. The frequencies can be obtained in the same method as the above and the results are shown in Table 5 and 6.

For this bridge, in the 1st, 2nd and 3rd modes the vertical bending vibration is predominant, but in the 4th and 5th modes the horizontal bending vibration. The torsional vibration will become more predominant in the higher frequencies than the 5 th, because a box girder has large torsional rigidity.

The field tests were also conducted in the same way as a) and the frequency was $3.44 \mathrm{c} / \mathrm{s}$ for the first mode as shown in Fig. 15.

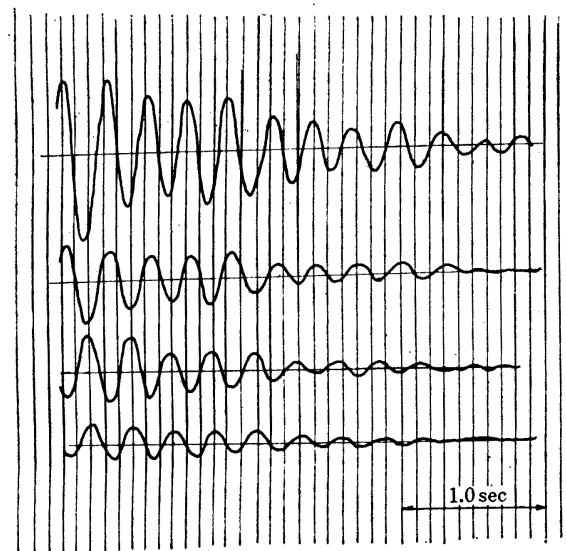

Fig. 15 Free vibration records (Nagara bridge) (deflection).

By comparing the measured values with three theoretical values $\left(f_{1, \mathrm{I}}\right)_{\mathrm{cal}}=3.360 \mathrm{c} / \mathrm{s},\left(f_{1}\right)_{\text {uncouple }}=$ $3.530 \mathrm{c} / \mathrm{s}$ and $\left(f_{1}\right)_{\text {straight }}=3.620 \mathrm{c} / \mathrm{s}$, the ratio between these theoretical values and measured ones are $98 \%, 103 \%$ and $105 \%$ respectively. Since the eccen- tric distances between the center of gravity and the shear center are small in this bridge, the coupled vibrations are not so appreciable. However, there will be some danger of over-estimating the rigidities, if we compute the frequencies about the uncoupled vibration or about a straight girder bridge.

In the calculation of the natural frequencies, the influence of a skewed support was neglected. It seems to be neccessary to consider the skewness of the middle support for this bridge though it is much difficult.

\section{Example of Double Coupled Vibrations.}

a) Jusoo Bypass Bridge in Osaka City.

This bridge is a three-span continuous curved girder bridge having a multiple plate I-girder as shown in Fig. 16. The floor system consists of reinforced concrete slab, but this bridge is not designed as a composite girder. Therefore, the statical tests were conducted to ensure the stiffness of this bridge by fully loading of nine 13.5 tons dump truck before the dynamical tests. From these tests the fact was that; (1) The concrete slab was considerably composite with the steel girder. The measured deflections were fairly coincided with the theoretical values calculated as composite girder by considering the effective width of a concrete slab and by putting $n=7$, except that the former was somewhat greater than the latter (about 10\%). This composite action might be produced by means of the slab clamps, but it is much difficult to know plainly the strength of a composite action. Accordingly, for the geometrical moments of inertia it is assumed to be a completely composite girder bridge.

Table 1 shows these quantities. In this case, it

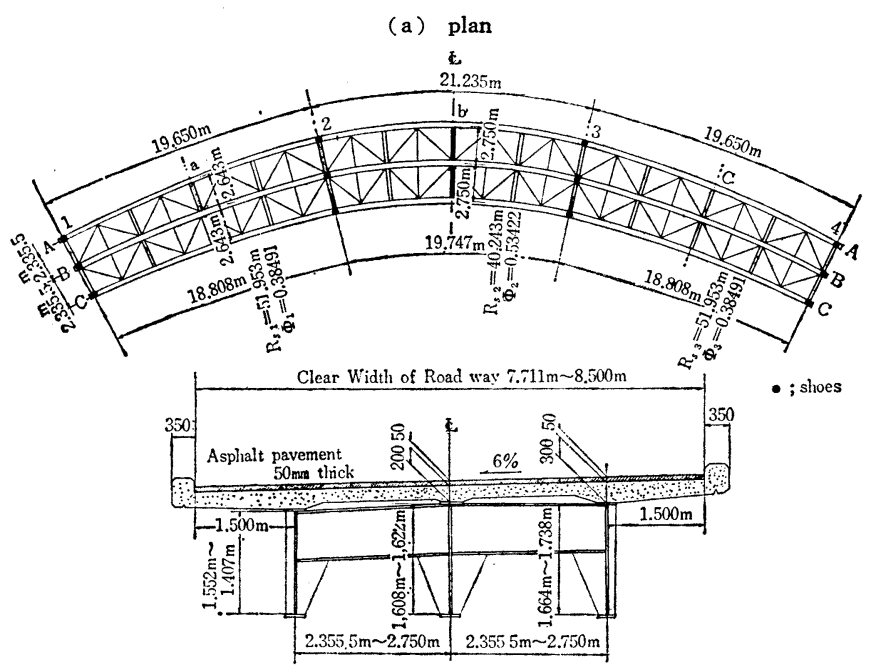

(b) typical cross-section

Fig. 16 Detail of Jusoo bridge. 
must be noted that the eccentricity of the center of gravity in the horizontal direction is negligible small so that we can put $S_{z}{ }^{*}=0$. Consequently, the circular frequency can be determined by the practical formula (111) on the coupled vibrations. These results are shown in Table $7 \sim 8$.

Table 7 Values $k_{i} \mathbf{L}_{1}$ and $p_{i, w w}{ }^{2}, p_{i, \beta \beta}{ }^{2}, \cdots$, etc. (Jusoo Br.)

\begin{tabular}{c|c||c|c|c|c}
\hline$i$ & $k_{i} L_{1}$ & $p_{i, w w w^{2}}$ & $p_{i, \beta w^{2}}$ & $p_{i, w \beta^{2}}$ & $p_{i, \beta \beta^{2}}$ \\
\hline 1 & 3.05 & $1.699 \times 10^{2}$ & $3.677 \times 10^{3}$ & $3.909 \times 10^{3}$ & $5.642 \times 10^{3}$ \\
2 & 3.53 & $3.080 \times 10^{3}$ & $3.892 \times 10^{3}$ & $4.371 \times 10^{3}$ & $9.261 \times 10^{3}$ \\
3 & 4.13 & $5.796 \times 10^{3}$ & $4.599 \times 10^{3}$ & $5.787 \times 10^{3}$ & $1.682 \times 10^{4}$ \\
4 & 5.77 & $2.206 \times 10^{4}$ & $1.581 \times 10^{3}$ & $2.142 \times 10^{4}$ & $6.201 \times 10^{4}$ \\
\hline
\end{tabular}

Table 8 Values $p_{i}, \mathrm{I} \sim \mathbb{I}^{2}$ and frequencies $f_{i}, \mathrm{I} \sim \mathbb{I}, \cdots,(\mathrm{c} / \mathrm{s})$ (Jusoo Br.)

\begin{tabular}{c|c|c||c|c}
\hline$i$ & $p_{i, \mathrm{I}^{2}}$ & $p_{i, \mathrm{I}^{2}}$ & $f_{i, \mathrm{I}}$ & $f_{i, \mathrm{II}}$ \\
\hline 1 & $1.594 \times 10^{3}$ & $5.725 \times 10^{3}$ & $6.353(1)$ & $12.05(3)$ \\
2 & $3.065 \times 10^{3}$ & $9.673 \times 10^{3}$ & $8.811(2)$ & $15.65(5)$ \\
3 & $5.790 \times 10^{3}$ & $1.801 \times 10^{4}$ & $12.110(4)$ & $21.36(6)$ \\
4 & $2.205 \times 10^{4}$ & $6.657 \times 10^{4}$ & $26.640(7)$ & 41.07 \\
\hline
\end{tabular}

Since the torsional rigidity is small in this bridge, the 1st and 2nd modes are predominant in the vertical bending vibration, while the torsional vibration actually appears in the 3rd mode.

The vibration tests were conducted by pulsating the bridge with an osillator. Fig. 17 shows the typicaly resonance curves, which clearly shows two resonant frequencies, whose mean value is $6.10 \mathrm{c} / \mathrm{s}$

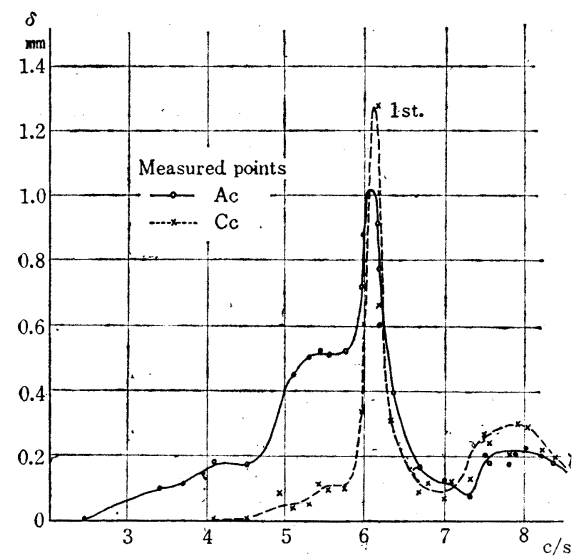

(a) deflection : $\delta$ (pulsating point AC)

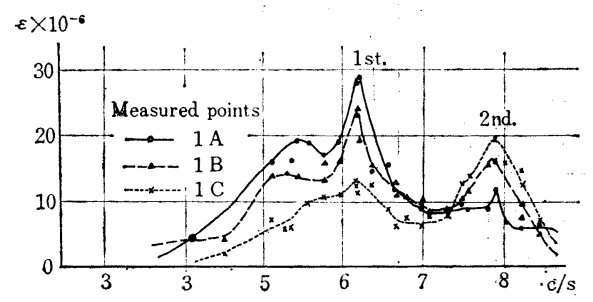

(a) stress : $\varepsilon$ (Pulsating point AC)

Fig. 17 Resonance curves. and $7.95 \mathrm{c} / \mathrm{s}$ for 1 st and $2 \mathrm{nd}$ mode respectively.

When the test results are compared with the theoretical values, the ratio between theoretical value and measured one is $104 \%$ and $111 \%$ for 1st and 2 nd mode respectively. The difference of both values seems to be caused by the assumption of the complete composite girder bridge.

In the lower modes where the bending vibrations are coupled with torsional one, the vertical bending vibration is predominant as shown in example 1 a), b) and $2 \mathrm{a}$ ), and the degree of coupling in lower modes is greater than that in higher modes. It is evident from the equation (101) that the values $p_{i, v v^{2}}, p_{i, w w^{2}}$ and $p_{i, \beta \beta^{2}}$ are proposional to the fourth power of the values $k_{i} L_{1}$, whereas the values $p_{i, 8 w}{ }^{2}$, $p_{i}, p_{i, w \beta}{ }^{2}, \cdots$, etc. are proposional to the second power of the values $k_{i} L_{1}$. Therefore, the difference between the values $p_{i, v v}{ }^{2}, p_{i, w w}{ }^{2}, p_{i, \beta \beta}{ }^{2}$ and $p_{i, w \beta}{ }^{2} P_{i, \beta w}{ }^{2}$, $\cdots$, etc. increase as the values of $k_{i} L_{1}$ become higher. On the other hand, the degree of coupling decrease in higher modes. Thus, the frequencies of coupled vibrations are always smaller than that of uncoupled in the lowest mode, that is to say, the rigidities of a curved girder bridge always tend to decrease.

This tendency can also be explained on the basis of the following analysis. If the statical downward deflection due to a unit force is denoted by the symbol $w_{s t}$, we know that the natural frequency in the fundamental mode can approximately be calculated by Rayleigh-Ritz's method as follows.

$$
f_{1, \mathrm{I}}=2 \pi \sqrt{\frac{g}{w_{s t}}}
$$

Then, the deflection of a curved gider bridge is usually much greater than that of a straight girder bridge having the identical span length and cross sectional dimensions. Therefore, this is the main reason why the lowest frequency decrease in coupled vibrations of curved girder bridge.

b) Nakano-Shima S-shaped Bridge on Hanshin Expess-Way.

The Nakano-Shima Bridge was planned as a threespan continuous $\mathrm{S}$-shaped curved girder bridge. Authors studied it to clarify the dynamical behaivours of such a paticular bridge ${ }^{16}$. So, the dynamical tests were conducted for a model girder before designing this bridge. The model bridge was fabricated on the scale of $1: 20$ under the consideration of the dimensional anlysis.

Fig. 18 shows the plane figure and the typical cross-section, where the radius of curvature of both side span is equal and has opposite directions to each other. The center span has also curvature, 
(a) cross section (mm)

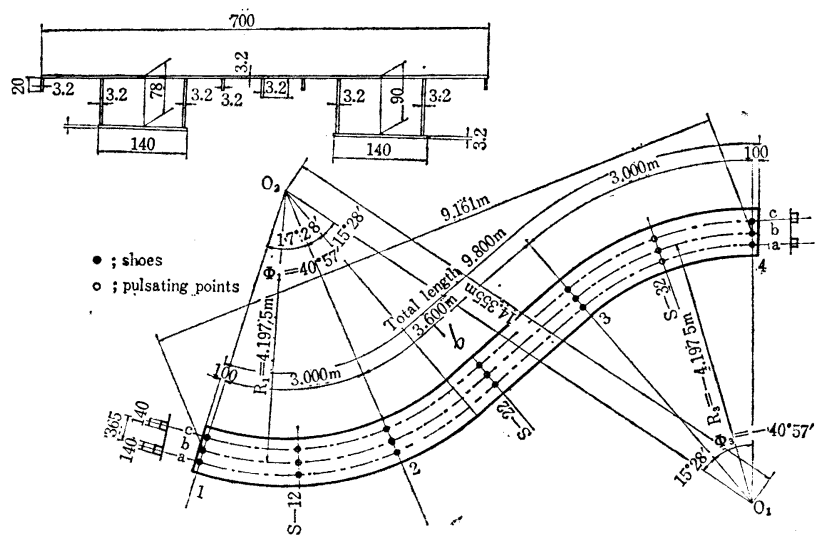

(b) plane (mm)

Fig. 18 Detail of model girder bridge.

but it may be regarded as a straight girder bridge having bisymmetrical cross-section because its curvature is much large.

Now, the bridge of this type can be considered to have the coupled vibrations of horizontal bending and twisting. The bending vibration in the vertical direction can be treated independently as previously described.

Table 9 shows the values $p_{i, v v}{ }^{2}, p_{i, w w^{2}}$ and $p_{i, \beta \beta^{2}}$ estimated from the practical formula (101). From these values, the natural frequencies can be obtained by Eq. (120) as shown in Table 10 .

Table 9 Values $k_{i} L_{1}$ and $p_{i, v v}{ }^{2}, p_{i, w w w}{ }^{2}, p_{i, \beta \beta}{ }^{2}$. (Nakano-shima Br.)

\begin{tabular}{c|c||c|c|c}
\hline$i$ & $k_{i} L_{1}$ & $p_{i, w w}{ }^{2}$ & $p_{i, v v^{2}}$ & $p_{i, \beta \beta}{ }^{2}$ \\
\hline 1 & 2.91 & $2.229 \times 10^{4}$ & $6.537 \times 10^{5}$ & $3.984 \times 10^{5}$ \\
2 & 3.51 & $4.703 \times 10^{4}$ & $1.379 \times 10^{6}$ & $5.657 \times 10^{5}$ \\
3 & 3.95 & $7.679 \times 10^{4}$ & $2.252 \times 10^{6}$ & $5.760 \times 10^{5}$ \\
4 & 5.72 & $3.411 \times 10^{5}$ & $1.001 \times 10^{7}$ & $1.853 \times 10^{6}$ \\
\hline
\end{tabular}

Table 10 Values $p_{i}, \mathbb{I} \sim \mathrm{m}^{2}$ and frequencies $f_{i}, 1 \sim \mathrm{II} .(\mathrm{c} / \mathrm{s})$ (Nakano-shima Br.)

\begin{tabular}{c|c|c||c|c|c}
\hline$i$ & $p_{i, \mathrm{I}^{2}}$ & $p_{i}, \mathrm{II}^{2}$ & $f_{i, \mathrm{I}}$ & $f_{i, \mathrm{II}}$ & $f_{i, \mathrm{II}}$ \\
\hline 1 & $3.8254 \times 10^{5}$ & $7.0059 \times 10^{6}$ & $23.76(1)$ & $98.42(5)$ & 133.2 \\
2 & $5.5567 \times 10^{5}$ & $1.4481 \times 10^{6}$ & $34.50(2)$ & 118.6 & 191.5 \\
3 & $5.7057 \times 10^{5}$ & $2.3399 \times 10^{6}$ & $44.09(3)$ & 120.2 & 243.5 \\
4 & $1.8406 \times 10^{5}$ & $1.0363 \times 10^{7}$ & $92.94(4)$ & 491.9 & 512.3 \\
\hline
\end{tabular}

It can be seen that the modes from the 1st to the 4th are bending vibration in the vertical direction. The coupled modes of bending vibration in the horizontal direction and torsional one are produced in the 5th mode for the first time.

The dynamical tests were conducted by pulsating the model girder by means of an electro-magnetic osillator. The measured data got by accelometers are shown in Fig. 19. The resonant frequencies are summarized together with their theoretical values

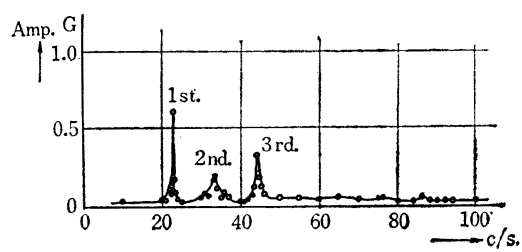

(a) pulsating point $\mathrm{S}-12 \mathrm{c}$

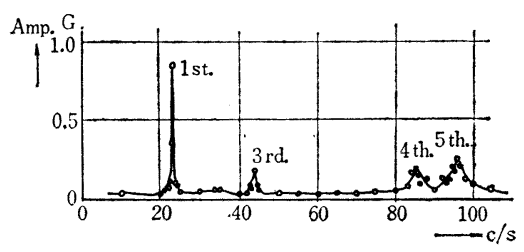

(b) pulsating point S-23c

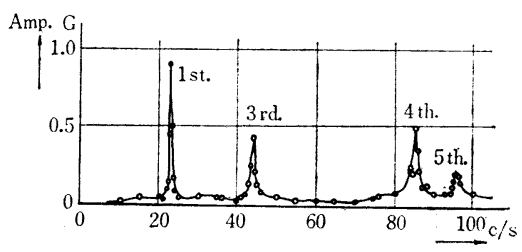

(c) pulsating point S-22 a

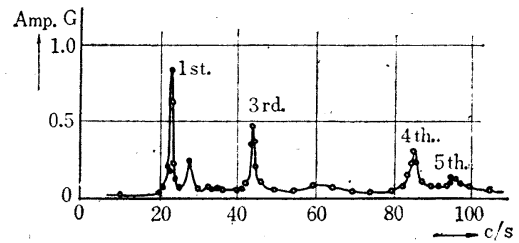

(d) pulsating point S-22 b

Fig. 19 Typical resonance curves. (Nakano-shima Bridge)

in Table 11. Fig. 20 shows the observed modes corresponding to these frequencies.

From the above data, it is clear that the theoretical values well coincide with the measured ones in the 1st, 2nd and 3rd modes. The deviation in the 
Table 11 Comparison of experimental values with theoretical ones. (c/s)

(Nakano-shima Br.)

\begin{tabular}{c|c|c|c}
\hline $\begin{array}{c}\text { Mode } \\
\text { order }\end{array}$ & $\begin{array}{c}\text { Experimental } \\
\text { values }\end{array}$ & $\begin{array}{c}\text { Theoretical } \\
\text { values }\end{array}$ & $\begin{array}{c}\text { Theoretical } \\
\text { values as } \\
\text { straight bridge }\end{array}$ \\
\hline 1st & 23 & 23.76 & 24.11 \\
2nd & 33 & 34.50 & 35.08 \\
3rd & 44 & 44.09 & 44.08 \\
4th & 85 & 92.94 & 93.17 \\
5th & 95 & 98.42 & 96.42 \\
\hline
\end{tabular}

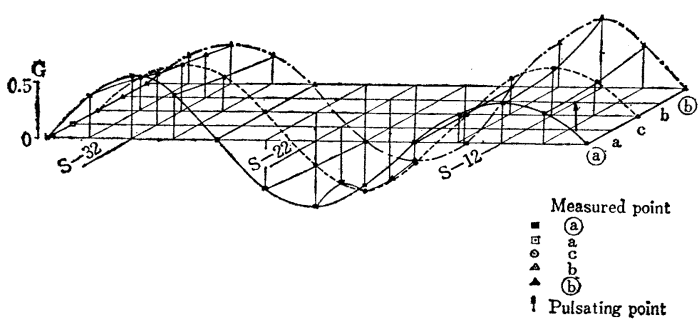

(a) 1 st mode $(23 \mathrm{c} / \mathrm{s})$

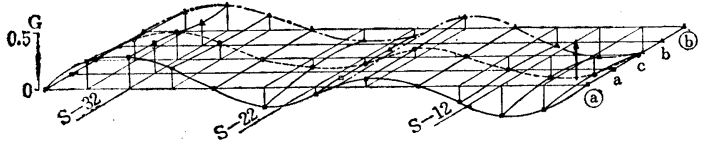

(b) 2 nd mode $(33 \mathrm{c} / \mathrm{s})$

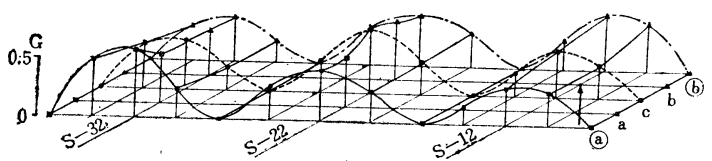

(c) $3 \mathrm{rd}$ mode $(44 \mathrm{c} / \mathrm{s})$

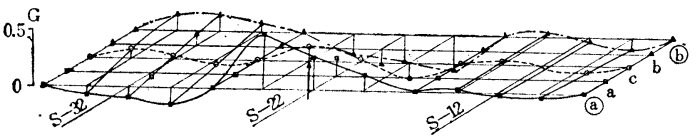

(d) 4 th mode $(85 \mathrm{c} / \mathrm{s})$

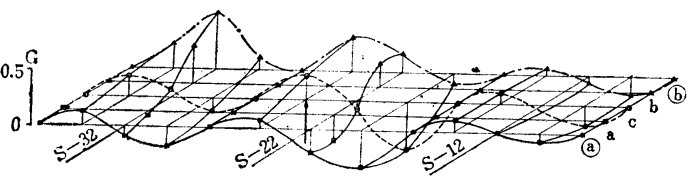

(e) 5 th mode $(95 \mathrm{c} / \mathrm{s})$

Fig. 20 Measured modes.

4 th and 5 th modes might be caused by the exceeding complexity of the coupled vibrations, since the 4 th and 5 th modes have almost equal frequencies. Besides, the curvature in the center span of existing model has more or less effect on the higher vibrations. The right column of Table 11 shows the frequencies computed with the assumption as a straight girder bridge.

\section{CONCLUSION.}

1. The practical equations for determining stress resultants and displacements of a curved girder bridge subjected to various kinds of statical forces have been derived.

2. From these equations the fundamental equations for the free vibration of a curved girder bridge have been presented.

3 . The approximate solution for the coupled vibrations of three modes were found by using Galerkin's method.

4. The natural frequencies are closely depend on the geometrical properties of a curved girder bridge as well as its materials.

5. The frequency equation for a curved girder bridge classified into,

a) coupled vibrations of three modes,

b) coupled vibrations of two modes,

c) un-coupled vibration.

6. The practical formulae for the three cases have been derived.

7. The above formulae have been verified by the field experimental studies and model test.

8. The lowest frequency of a curved girder bridge is always lower than the value estimated as an uncoupled vibration or as a straight girder birdge. The rigidities of a curved girder bridge are apparently much smaller than those of a straight girder bridge having identical span length and cross-section.

9. It may be concluded that in a S-shaped curved girder bridge like this example, the vertical bending vibration is independent, while the horizontal bending vibration coupled with torsional vibration, which has also been verified by the experimental studies.

10. In a curved girder bridge having a concrete deck, though there are a few point remaining to be solved concerning with the estimation of stiffness, it will be sufficient reasonable to calculate its frequencies as a composite girder bridge.

\section{ACKNOWLEDGEMENT}

In writing this report, the authors are grateful to The Japan Ministry of Education for the scientic research funds. Our thanks are also due to; Japan Ministry of Construction, Osaka City Office, Japan High-Way Public Cooperation, Hanshin High-Way Public Cooperaton, Matsuo Bridge Co. Ltd., Japan Bridge Co. Ltd., and Kawasaki Heavy Industrial Co. Ltd.. Moreover, the authors heartily wish to acknowledge the valuable encouragements by; Prof. Dr. Eng., Yoshio Tachibana and all the members of the Bridge Engineering Laboratory of Osaka City University. H. Nakai, one of the authors, is indebted personally to The Sakkokai Foundation for the subsidiary assistance in preparing this paper.

\section{Reference}

1) G. Schumpich : Beitrag zur Kinetic und Statik ebener Stabwerke mit gekrumten stäben. Österreiches Inge. 
nieur Archiv., 1957, s. 194 225

2) A. Hirai and Y. Fukazawa : Analysis of Dynamical Problem of Curved Girder Bridges. The abstract of 19th Annual Conference for Japan Soc. of Civ. Eng., May 1964.

3) H. Yonezawa : Moments and Free Vibration in Curved Girder Bridge., Proceeding of A.S.C.E., EM 1, Feb. 1962, p. 1 21.

4) S.P. Timoshenko : Vibration Problem in Engineering., 3rd Ed., 1955.

5) J.M. Gere and Y.K. Lin : Coupled Vibrations of ThinWalled Beams of Open Cross Section., Journal of Applied Mechanics., Sept. 1958, p. 373 378.

6) A.E.H. Love : Mathematical Theory of Elasticity., 4th Ed., 1927

7) M. Kuranishi : Theory of Elasticity (inJapanese). 1943, Japan Soc. of Mechanics.

8) I. Konishi and S. Komatsu : On Fundamental Theory of Thin-Walled Curved Girder. Trans of Japan Soc. of Civ. Eng., No. 87, Nov., 1962 p. 35 48.

9) S. Kuranishi : Analysis of Thin Walled Curved Beam. Trans. of Japan Soc. of Civ. Eng., No. 108, Aug, 1964 p. $7 \sim 12$.
10) Y. Fukazawa : Fundamental Theory on Statical Analysis of Thin-walled Curved Bars., Trans. of Japan Soc. of Civ. Eng. No. 110, Oct. 1964. p. 30 51.

11) I. Konishi and S. Komatsu: Three dimensional Analysis of Simply Supported Curved Girder Bridges. Trans. of Japan Soc. of Civ. Eng. No. 90, Feb. 1963 p. $11 \sim 28$.

12) I. Konishi and S. Komatsu : Three Dimensional Analysis for Continuous Curved Girder Bridges. Trans. of Japan Soc. of Civ. Eng., No. 91 Mar. 1963. p. 13 24.

13) S. Komatsu : Structual Analysis for Continuous SShaped Girder Bridge., Proceeding of the thirteenth Japan National Congress for Applied Mechanics., 1963. p. $17 \sim 22$.

14) I. Konishi and S. Komatsu : On the Vibration Characteristic of Gerber Beam., Journal of Japan Soc. of Civ. Eng., Sept. 1953, p. $407 \sim 412$.

15) G.L. Rogers : Dynamics of Flamed Structure. 1958.

16) H. Gotō, S. Komatsu, K. Iwamoto, S. Emi and M. Funakawa: Model Dynamical Studies on S-Shaped Curved Girder Bridge., The abstract of 19th Annual Conference for Japan Soc. of Civ. Eng., May. 1964.

APPENDIX

\begin{tabular}{|c|c|c|c|c|c|c|c|c|}
\hline$l_{r} k_{i} L_{1}$ & $\psi_{i, r}$ & $\chi_{i, r}$ & $\zeta_{i, r}$ & $\varphi_{i, r}$ & $\mu_{i, r}$ & $\kappa_{i, r}$ & $v_{i, r}$ & $\theta_{i, r}$ \\
\hline 0.1 & $3.3332 \times 10^{-1}$ & $6.6668 \times 10^{-1}$ & $3.3335 \times 10^{-1}$ & $2.0000 \times 10^{1}$ & $1.4842 \times 10^{-5}$ & $3.1468 \times 10^{-5}$ & $8.9455 \times 10^{-4}$ & $1.5450 \times 10^{-3}$ \\
\hline 0.2 & $3.3334 \times 10^{-1}$ & $6.6667 \times 10^{-1}$ & $3.3337 \times 10^{-1}$ & $1.0000 \times 10^{1}$ & $1.3046 \times 10^{-5}$ & $2.7057 \times 10^{-5}$ & $3.5560 \times 10^{-3}$ & $6.2222 \times 10^{-3}$ \\
\hline 0.3 & $3.3337 \times 10^{-1}$ & $6.6667 \times 10^{-1}$ & $3.3350 \times 10^{-1}$ & 6.6677 & $6.8303 \times 10^{-5}$ & $1.3327 \times 10^{-4}$ & $8.0014 \times 10^{-3}$ & $1.4002 \times 10^{-2}$ \\
\hline 0.4 & $3.3344 \times 10^{-1}$ & $6.6687 \times 10^{-1}$ & $3.3386 \times 10^{-1}$ & 5.0025 & $2.1669 \times 10^{-4}$ & $4.2044 \times 10^{-4}$ & $1.4229 \times 10^{-2}$ & $2.4903 \times 10^{-2}$ \\
\hline 0.5 & $3.3359 \times 10^{-1}$ & $6.6693 \times 10^{-1}$ & $3.3462 \times 10^{-1}$ & 4.0049 & $5.2974 \times 10^{-4}$ & $1.0264 \times 10^{-3}$ & $2.2249 \times 10^{-2}$ & $3.8941 \times 10^{-2}$ \\
\hline 0.6 & $3.3387 \times 10^{-1}$ & $6.6722 \times 10^{-1}$ & $3.3600 \times 10^{-1}$ & 3.3417 & $1.1001 \times 10^{-3}$ & $2.1313 \times 10^{-3}$ & $3.2079 \times 10^{-2}$ & $5.6157 \times 10^{-2}$ \\
\hline 0.7 & $3.3432 \times 10^{-1}$ & $6.6769 \times 10^{-1}$ & $3.3828 \times 10^{-1}$ & 2.8705 & $2.0425 \times 10^{-3}$ & $3.9579 \times 10^{-3}$ & $4.3756 \times 10^{-2}$ & $7.6619 \times 10^{-2}$ \\
\hline 0.8 & $3.3502 \times 10^{-1}$ & $6.6841 \times 10^{-1}$ & $3.4180 \times 10^{-1}$ & 2.5200 & $3.4964 \times 10^{-3}$ & $6.7759 \times 10^{-3}$ & $5.7336 \times 10^{-2}$ & $1.0044 \times 10^{-1}$ \\
\hline 0.9 & $3.3604 \times 10^{-1}$ & $6.6946 \times 10^{-1}$ & $3.4695 \times 10^{-1}$ & 2.2508 & $5.6286 \times 10^{-3}$ & $1.0910 \times 10^{-2}$ & $7.2909 \times 10^{-2}$ & $1.2780 \times 10^{-1}$ \\
\hline 1.0 & $3.3748 \times 10^{-1}$ & $6.7094 \times 10^{-1}$ & $3.5423 \times 10^{-1}$ & 2.0393 & $8.6393 \times 10^{-3}$ & $1.6749 \times 10^{-2}$ & $9.0608 \times 10^{-2}$ & $1.5897 \times 10^{-1}$ \\
\hline 1.1 & $3.3943 \times 10^{-1}$ & $6.7296 \times 10^{-1}$ & $3.6419 \times 10^{-1}$ & 1.8708 & $1.2770 \times 10^{-2}$ & $2.4763 \times 10^{-1}$ & $1.1062 \times 10^{-1}$ & $1.9431 \times 10^{-1}$ \\
\hline 1.2 & $3.4202 \times 10^{-1}$ & $6.7563 \times 10^{-1}$ & $3.7755 \times 10^{-1}$ & 1.7354 & $1.8314 \times 10^{-2}$ & $3.5528 \times 10^{-2}$ & $1.3322 \times 10^{-1}$ & $2.3436 \times 10^{-1}$ \\
\hline 1.3 & $3.4540 \times 10^{-1}$ & $6.7912 \times 10^{-1}$ & $3.9516 \times 10^{-1}$ & 1.6266 & $2.5637 \times 10^{-2}$ & $4.9758 \times 10^{-2}$ & $1.5877 \times 10^{-1}$ & $2.7985 \times 10^{-1}$ \\
\hline 1.4 & $3.4974 \times 10^{-1}$ & $6.8358 \times 10^{-1}$ & $4.1810 \times 10^{-1}$ & 1.5399 & $3.5203 \times 10^{-2}$ & $6.8363 \times 10^{-2}$ & $1.8776 \times 10^{-1}$ & $3.3177 \times 10^{-3}$ \\
\hline 1.5 & $3.5525 \times 10^{-1}$ & $6.8925 \times 10^{-1}$ & $4.4776 \times 10^{-1}$ & 1.4722 & $4.7607 \times 10^{-2}$ & $9.2518 \times 10^{-2}$ & $2.2089 \times 10^{-1}$ & $3.9149 \times 10^{-1}$ \\
\hline 1.6 & $3.6217 \times 10^{-1}$ & $6.9638 \times 10^{-1}$ & $4.8595 \times 10^{-1}$ & 1.4214 & $6.3639 \times 10^{-2}$ & $1.2378 \times 10^{-1}$ & $2.5910 \times 10^{-1}$ & $4.6086 \times 10^{-3}$ \\
\hline 1.7 & $3.7084 \times 10^{-1}$ & $7.0528 \times 10^{-1}$ & $5.3510 \times 10^{-1}$ & 1.3864 & $8.4365 \times 10^{-2}$ & $1.6426 \times 10^{-1}$ & $3.0366 \times 10^{-1}$ & $5.4246 \times 10^{-1}$ \\
\hline 1.8 & $3.8165 \times 10^{-1}$ & $7.1638 \times 10^{-1}$ & $5.9855 \times 10^{-1}$ & 1.3667 & $1.1126 \times 10^{-1}$ & $2.1690 \times 10^{-1}$ & $3.5640 \times 10^{-1}$ & $6.3938 \times 10^{-1}$ \\
\hline 1.9 & $3.9514 \times 10^{-1}$ & $7.3021 \times 10^{-1}$ & $6.8101 \times 10^{-1}$ & 1.3627 & $1.4644 \times 10^{-1}$ & $2.8587 \times 10^{-1}$ & $4.1987 \times 10^{-1}$ & $7.5826 \times 10^{-1}$ \\
\hline 2.0 & $4.1201 \times 10^{-1}$ & $7.4749 \times 10^{-1}$ & $7.8932 \times 10^{-1}$ & 1.3755 & $1.9297 \times 10^{-1}$ & $3.7730 \times 10^{-1}$ & $4.9782 \times 10^{-1}$ & $9.0504 \times 10^{-1}$ \\
\hline 2.1 & $4.3325 \times 10^{-1}$ & $7.6919 \times 10^{-1}$ & $9.3374 \times 10^{-1}$ & 1.4071 & $2.5552 \times 10^{-1}$ & $5.0049 \times 10^{-1}$ & $5.9584 \times 10^{-1}$ & 1.0914 \\
\hline 2.2 & $4.6023 \times 10^{-1}$ & $7.9671 \times 10^{-1}$ & 1.1302 & I. 4612 & $3.4139 \times 10^{-1}$ & $6.7002 \times 10^{-1}$ & $7.2259 \times 10^{-1}$ & 1.3346 \\
\hline 2.3 & $4.9498 \times 10^{-1}$ & $8.3208 \times 10^{-1}$ & 1.4048 & 1.5436 & $4.6260 \times 10^{-1}$ & $9.0986 \times 10^{-1}$ & $8.9210 \times 10^{-1}$ & 1.6626 \\
\hline 2.4 & $5.4064 \times 10^{-1}$ & $8.7845 \times 10^{-1}$ & 1.8022 & 1.6634 & $6.3993 \times 10^{-1}$ & 1.2615 & 1.1283 & 2.1233 \\
\hline 2.5 & $6.0226 \times 10^{-1}$ & $9.4089 \times 10^{-1}$ & 2.4043 & 1.8362 & $9.1189 \times 10^{-1}$ & 1.8020 & 1.4747 & 2.8037 \\
\hline 2.6 & $6.8865 \times 10^{-1}$ & 1.0282 & 3.3755 & 2.0892 & 1.3563 & 2.6869 & 2.0179 & 3.8770 \\
\hline 2.7 & $8.1660 \times 10^{-1}$ & 1.1572 & 5.0859 & 2.4749 & 2.1497 & 4.2693 & 2.9514 & 5.7300 \\
\hline 2.8 & 1.0225 & 1.3643 & 8.5194 & 3.1073 & 3.7660 & 7.4969 & 4.7855 & 9.3832 \\
\hline 2.9 & 1.4032 & 1.7464 & $1.7074 \times 10^{1}$ & 4.2901 & 7.8558 & $1.5671 \times 10^{-1}$ & 9.2675 & $1.8331 \times 10^{1}$ \\
\hline 3.0 & 2.3288 & 2.6734 & $4.9812 \times 10^{1}$ & 7.1860 & $2.3765 \times 10^{-1}$ & $4.7483 \times 10^{-1}$ & $2.6114 \times 10^{-1}$ & $5.2006 \times 10^{1}$ \\
\hline 3.1 & 7.7288 & 8.0751 & $5.7797 \times 10^{2}$ & $2.4140 \times 10^{1}$ & $2.8515 \times 10^{2}$ & $5.7025 \times 10^{2}$ & $2.9291 \times 10^{2}$ & $5.8558 \times 10^{2}$ \\
\hline 3.2 & -5.3789 & -5.0307 & $2.9305 \times 10^{2}$ & $-1.7049 \times 10^{1}$ & $1.4925 \times 10^{2}$ & $2.9843 \times 10^{2}$ & $1.4391 \times 10^{2}$ & $2.8756 \times 10^{2}$ \\
\hline 3.3 & -1.9434 & -1.5931 & $3.9758 \times 10^{1}$ & -6.2655 & $2.0887 \times 10^{1}$ & $4.1701 \times 10^{1}$ & $1.8996 \times 10^{1}$ & $3.7711 \times 10^{1}$ \\
\hline 3.4 & -1.1706 & $-8.1797 \times 10^{-1}$ & $1.4872 \times 10^{1}$ & -3.8464 & 8.0636 & $1.6043 \times 10^{1}$ & 6.9553 & $1.3607 \times 10^{1}$ \\
\hline 3.5 & $-8.3178 \times 10^{-1}$ & $-4.7651 \times 10^{-1}$ & 7.6710 & 2.7903 & 4.2999 & 8.5028 & 3.5408 & 6.7527 \\
\hline 3.6 & $-6.4291 \times 10^{-1}$ & $-2.8472 \times 10^{-1}$ & 4.6342 & -2.2051 & 2.6942 & 5.2771 & 2.1343 & 3.9121 \\
\hline 3.7 & $-5.2347 \times 10^{-1}$ & $-1.6202 \times 10^{-1}$ & 3.0706 & -1.8379 & 1.8609 & 3.5941 & 1.4307 & 2.4748 \\
\hline 3.8 & $-4.4188 \times 10^{-1}$ & $-7.6771 \times 10^{-2}$ & 2.1576 & -1.5896 & 1.3730 & 2.5995 & 1.0348 & 1.6497 \\
\hline 3.9 & $-3.8320 \times 10^{-1}$ & $-1.4019 \times 10^{-2}$ & 1.5752 & -1.4135 & 1.0632 & 1.9584 & 7.9422 & 1.1317 \\
\hline 4.0 & $-3.3950 \times 10^{-1}$ & $3.4245 \times 10^{-2}$ & 1.1779 & -1.2847 & $8.5519 \times 10^{-1}$ & 1.5174 & $6.4061 \times 10^{-1}$ & $7.8339 \times 10^{-1}$ \\
\hline
\end{tabular}




\begin{tabular}{|c|c|c|c|c|c|c|c|c|}
\hline$l_{r} k_{i} L_{1}$ & $\psi_{i, r}$ & $\chi_{i, r}$ & $\zeta_{i, r}$ & $\varphi_{i, r}$ & $\mu_{i, r}$ & $\kappa_{i, r}$ & $v_{i, r}$ & $\theta_{i, r}$ \\
\hline 4.1 & $-3.0615 \times 10^{-1}$ & $7.2700 \times 10^{-2}$ & $8.9166 \times 10^{-1}$ & -1.1889 & $7.0984 \times 10^{-1}$ & 1.1978 & $5.3960 \times 10^{-1}$ & $5.3533 \times 10^{-1}$ \\
\hline 4.2 & $-2.8032 \times 10^{-1}$ & $1.0427 \times 10^{-1}$ & $6.7539 \times 10^{-1}$ & -1.1173 & $6.0562 \times 10^{-1}$ & $9.5571 \times 10^{-1}$ & $4.7259 \times 10^{-1}$ & $3.4934 \times 10^{-1}$ \\
\hline 4.3 & $-2.6015 \times 10^{-1}$ & $1.3091 \times 10^{-1}$ & $5.0466 \times 10^{-1}$ & -1.0644 & $5.2987 \times 10^{-1}$ & $7.6481 \times 10^{-1}$ & $4.2887 \times 10^{-1}$ & $2.0283 \times 10^{-1}$ \\
\hline 4.4 & $-2.4441 \times 10^{-1}$ & $1.5394 \times 10^{-1}$ & $3.6396 \times 10^{-1}$ & -1.0263 & $4.7488 \times 10^{-1}$ & $6.0837 \times 10^{-1}$ & $4.0208 \times 10^{-1}$ & $8.1573 \times 10^{-1}$ \\
\hline 4.5 & $-2.3227 \times 10^{-1}$ & $1.7436 \times 10^{-1}$ & $2.4282 \times 10^{-1}$ & -1.0008 & $4.3583 \times 10^{-1}$ & $4.7509 \times 10^{-1}$ & $3.8840 \times 10^{-1}$ & $2.4020 \times 10^{-1}$ \\
\hline 4.6 & $-2.2314 \times 10^{-1}$ & $1.9290 \times 10^{-1}$ & $1.3369 \times 10^{-1}$ & $-9.8624 \times 10^{-1}$ & $4.0972 \times 10^{-1}$ & $3.5683 \times 10^{-1}$ & $3.8559 \times 10^{-1}$ & $1.2093 \times 10^{-1}$ \\
\hline 4.7 & $-2.1665 \times 10^{-1}$ & $2.1017 \times 10^{-1}$ & $3.0586 \times 10^{-2}$ & $-9.8188 \times 10^{-1}$ & $3.9483 \times 10^{-1}$ & $2.4794 \times 10^{-1}$ & $3.9252 \times 10^{-1}$ & $-2.1472 \times 10^{-1}$ \\
\hline 4.8 & $-2.1256 \times 10^{-1}$ & $2.2666 \times 10^{-1}$ & $-7.1711 \times 10^{-2}$ & $-9.8739 \times 10^{-1}$ & $3.9039 \times 10^{-1}$ & $1.4085 \times 10^{-1}$ & $4.0896 \times 10^{-1}$ & $-3.1034 \times 10^{-1}$ \\
\hline 4.9 & $-2.1077 \times 10^{-1}$ & $2.4285 \times 10^{-1}$ & $-1.7834 \times 10^{-1}$ & -1.0030 & $3.9649 \times 10^{-1}$ & $3.2428 \times 10^{-2}$ & $4.3545 \times 10^{-1}$ & $-4.1282 \times 10^{-1}$ \\
\hline 5.0 & $-2.1126 \times 10^{-1}$ & $2.5918 \times 10^{-1}$ & $-2.9501 \times 10^{-1}$ & -1.2094 & $4.1407 \times 10^{-1}$ & $-8.3744 \times 10^{-2}$ & $4.7342 \times 10^{-1}$ & $-5.2783 \times 10^{-1}$ \\
\hline 5.1 & $-2.1418 \times 10^{-1}$ & $2.7614 \times 10^{-1}$ & $-4.2878 \times 10^{-1}$ & -1.0679 & $4.4519 \times 10^{-1}$ & $-2.1460 \times 10^{-1}$ & $5.2539 \times 10^{-1}$ & $-6.6257 \times 10^{-1}$ \\
\hline 5.2 & $-2.1980 \times 10^{-1}$ & $2.9430 \times 10^{-1}$ & $-5.8925 \times 10^{-1}$ & -1.1209 & $4.9341 \times 10^{-1}$ & $-3.6945 \times 10^{-1}$ & $5.9552 \times 10^{-1}$ & $-8.2687 \times 10^{-1}$ \\
\hline 5.3 & $-2.2859 \times 10^{-1}$ & $3.1437 \times 10^{-1}$ & $-7.9036 \times 10^{-1}$ & -1.1916 & $5.6461 \times 10^{-1}$ & $-5.6177 \times 10^{-1}$ & $6.9039 \times 10^{-1}$ & -1.0352 \\
\hline 5.4 & $-2.4131 \times 10^{-1}$ & $3.3729 \times 10^{-1}$ & -1.0538 & -1.2850 & $6.6860 \times 10^{-1}$ & $-8.1250 \times 10^{-1}$ & $8.2078 \times 10^{-1}$ & -1.3098 \\
\hline 5.5 & $-2.5919 \times 10^{-1}$ & $3.6445 \times 10^{-1}$ & -1.4155 & -1.4092 & $8.2219 \times 10^{-1}$ & -1.1563 & 1.0049 & -1.6880 \\
\hline 5.6 & $-2.8420 \times 10^{-1}$ & $3.9797 \times 10^{-1}$ & -1.9388 & -1.5767 & 1.0557 & -1.6546 & 1.2751 & -2.2352 \\
\hline 5.7 & $-3.1976 \times 10^{-1}$ & $4.4137 \times 10^{-1}$ & -2.7458 & -1.8092 & 1.4281 & -2.4261 & 1.6940 & -3.0766 \\
\hline 5.8 & $-3.7214 \times 10^{-1}$ & $5.0103 \times 10^{-1}$ & -4.0963 & -2.1463 & 2.0658 & -3.7242 & 2.3945 & -4.4785 \\
\hline 5.9 & $-4.5426 \times 10^{-1}$ & $5.8995 \times 10^{-1}$ & -6.6296 & -2.6692 & 3.2820 & -6.1753 & 3.7024 & -7.0930 \\
\hline 6.0 & $-5.9731 \times 10^{-1}$ & $7.3939 \times 10^{-1}$ & $-1.2293 \times 10^{1}$ & -3.5739 & 6.0345 & $-1.1696 \times 10^{1}$ & 6.6073 & $-1.2899 \times 10^{1}$ \\
\hline 6.1 & $-9.0067 \times 10^{-1}$ & 1.0488 & $-2.9627 \times 10^{1}$ & -5.4851 & $1.4543 \times 10^{1}$ & $-2.8726 \times 10^{1}$ & $1.5428 \times 10^{1}$ & $-3.0535 \times 10^{1}$ \\
\hline 6.2 & -1.9418 & 2.0957 & $-1.4434 \times 10^{2}$ & $-1.2031 \times 10^{1}$ & $7.1375 \times 10^{1}$ & $-1.4240 \times 10^{2}$ & $7.3310 \times 10^{1}$ & $-1.4629 \times 10^{2}$ \\
\hline 6.3 & 9.4399 & -9.2804 & $-3.5368 \times 10^{3}$ & $5.9479 \times 10^{1}$ & $1.7733 \times 10^{3}$ & $-3.5462 \times 10^{3}$ & $1.7638 \times 10^{3}$ & $-3.5273 \times 10^{3}$ \\
\hline 6.4 & 1.3401 & -1.1752 & $-7.3113 \times 10^{1}$ & 8.5834 & $3.7396 \times 10^{1}$ & $-7.4453 \times 10^{1}$ & $3.6065 \times 10^{1}$ & $-7.1778 \times 10^{1}$ \\
\hline 6.5 & $7.1470 \times 10^{-1}$ & $-5.4457 \times 10^{-1}$ & $-2.1100 \times 10^{1}$ & 4.6516 & $1.1077 \times 10^{t}$ & $-2.1815 \times 10^{1}$ & $1.0378 \times 10^{1}$ & $-2.0391 \times 10^{1}$ \\
\hline 6.6 & $4.8593 \times 10^{-1}$ & $-3.1062 \times 10^{-1}$ & -9.7876 & 3.2126 & 5.3069 & $-1.0274 \times 10^{1}$ & 4.8447 & -9.3063 \\
\hline 6.7 & $3.6830 \times 10^{-1}$ & $-1.8785 \times 10^{-1}$ & -5.5763 & 2.4725 & 3.1445 & -5.9446 & 2.8074 & -5.2122 \\
\hline 6.8 & $2.9729 \times 10^{-1}$ & $-1.1169 \times 10^{-1}$ & -3.5587 & 2.0261 & 2.1038 & -3.8560 & 1.8450 & -3.2652 \\
\hline 6.9 & $2.5026 \times 10^{-1}$ & $-5.9451 \times 10^{-1}$ & -2.4360 & 1.7308 & 1.5241 & -2.6862 & 1.3197 & -2.1891 \\
\hline 7.0 & $2.1718 \times 10^{-1}$ & $-2.1073 \times 10^{-1}$ & -1.7448 & 1.5239 & 1.1689 & -1.9620 & 1.0050 & -1.5308 \\
\hline 7.1 & $1.9298 \times 10^{-2}$ & $8.5831 \times 10^{-3}$ & -1.2866 & 1.3735 & $9.3663 \times 10^{-1}$ & -1.4795 & $8.0437 \times 10^{-1}$ & -1.0964 \\
\hline 7.2 & $1.7479 \times 10^{-1}$ & $3.2430 \times 10^{-2}$ & $-9.6428 \times 10^{-1}$ & 1.2615 & $7.7755 \times 10^{-1}$ & -1.1391 & $6.7109 \times 10^{-1}$ & $-7.9207 \times 10^{-1}$ \\
\hline 7.3 & $1.6089 \times 10^{-1}$ & $5.2247 \times 10^{-2}$ & $-7.2604 \times 10^{-1}$ & 1.1772 & $6.6521 \times 10^{-1}$ & $-8.8693 \times 10^{-1}$ & $5.8047 \times 10^{-1}$ & $-5.6748 \times 10^{-1}$ \\
\hline 7.4 & $1.5020 \times 10^{-1}$ & $6.9193 \times 10^{-2}$ & $-5.4175 \times 10^{-1}$ & 1.1139 & $5.8446 \times 10^{-1}$ & $-6.9195 \times 10^{-1}$ & $5.1852 \times 10^{-1}$ & $-3.9367 \times 10^{-1}$ \\
\hline 7.5 & $1.4200 \times 10^{-1}$ & $8.4060 \times 10^{-2}$ & $-3.9287 \times 10^{-1}$ & 1.0672 & $5.2652 \times 10^{-1}$ & $-5.3487 \times 10^{-1}$ & $4.7698 \times 10^{-1}$ & $-2.5279 \times 10^{-1}$ \\
\hline 7.6 & $1.3581 \times 1 .^{-1}$ & $9.7423 \times 10^{-2}$ & $-2.6719 \times 10^{-1}$ & 1.0341 & $4.8498 \times 10^{1}$ & $-4.0300 \times 10^{-1}$ & $4.5083 \times 10^{-1}$ & $-1.3312 \times 10^{-1}$ \\
\hline 7.7 & $1.3131 \times 10^{-1}$ & $1.0971 \times 10^{-1}$ & $-1.5616 \times 10^{-1}$ & 1.0129 & $4.5719 \times 10^{-1}$ & $-2.8747 \times 10^{-1}$ & $4.3703 \times 10^{-1}$ & $-2.6432 \times 10^{-2}$ \\
\hline 7.8 & $1.2829 \times 10^{-1}$ & $1.2128 \times 10^{-1}$ & $-5.3294 \times 10^{-2}$ & 1.0023 & $4.4082 \times 10^{-1}$ & $-1.8158 \times 10^{-1}$ & $4.3389 \times 10^{-1}$ & $7.3565 \times 10^{-2}$ \\
\hline 7.9 & $1.2662 \times 10^{-1}$ & $1.3241 \times 10^{-1}$ & $-4.6841 \times 10^{-2}$ & 1.0018 & $4.3485 \times 10^{-1}$ & $-7.9782 \times 10^{-1}$ & $4.4068 \times 10^{-1}$ & $1.7217 \times 10^{-1}$ \\
\hline 8.0 & $1.2626 \times 10^{-1}$ & $1.4338 \times 10^{-1}$ & $1.4932 \times 10^{-1}$ & 1.0114 & $4.3912 \times 10^{-1}$ & $2.3057 \times 10^{-1}$ & $4.5751 \times 10^{-1}$ & $2.7440 \times 10^{-1}$ \\
\hline 8.1 & $1.2721 \times 10^{-1}$ & $1.5446 \times 10^{-1}$ & $2.5951 \times 10^{-1}$ & 1.0317 & $4.5430 \times 10^{-1}$ & $1.3229 \times 10^{-1}$ & $4.8530 \times 10^{-1}$ & $3.8566 \times 10^{-1}$ \\
\hline 8.2 & $1.2957 \times 10^{-1}$ & $1.6592 \times 10^{-1}$ & $3.8379 \times 10^{-1}$ & 1.0636 & $4.8203 \times 10^{-1}$ & $2.5422 \times 10^{-1}$ & $5.2600 \times 10^{-1}$ & $5.1239 \times 10^{-1}$ \\
\hline 8.3 & $1.3349 \times 10^{-1}$ & $1.7809 \times 10^{-1}$ & $5.3050 \times 10^{-1}$ & 1.1089 & $5.2527 \times 10^{-1}$ & $3.9701 \times 10^{-1}$ & $5.8288 \times 10^{-1}$ & $6.6311 \times 10^{-1}$ \\
\hline 8.4 & $1.3925 \times 10^{-1}$ & $1.9139 \times 10^{-1}$ & $7.1147 \times 10^{-1}$ & 1.1706 & $5.8892 \times 10^{-1}$ & $5.7222 \times 10^{-1}$ & $6.6126 \times 10^{-1}$ & $8.4993 \times 10^{-1}$ \\
\hline 8.5 & $1.4729 \times 10^{-1}$ & $2.0635 \times 10^{-1}$ & $9.4462 \times 10^{-1}$ & 1.2528 & $6.8104 \times 10^{-1}$ & $7.9733 \times 10^{-1}$ & $7.6974 \times 10^{-1}$ & 1.0912 \\
\hline 8.6 & $1.5829 \times 10^{-1}$ & $2.2374 \times 10^{-1}$ & 1.2588 & 1.3620 & $8.1519 \times 10^{-1}$ & 1.1005 & $9.2265 \times 10^{-1}$ & 1.4164 \\
\hline 8.7 & $1.7334 \times 10^{-1}$ & $2.4474 \times 10^{-1}$ & 1.7036 & 1.5087 & 1.0152 & 1.5303 & 1.1450 & 1.8764 \\
\hline 8.8 & $1.9424 \times 10^{-1}$ & $2.7121 \times 10^{-1}$ & 2.3710 & 1.7099 & 1.3258 & 2.1768 & 1.4834 & 2.5647 \\
\hline 8.9 & $2.2423 \times 10^{-1}$ & $3.0644 \times 10^{-1}$ & 3.4479 & 1.9926 & 1.8386 & 3.2237 & 2.0327 & 3.6717 \\
\hline 9.0 & $2.6958 \times 10^{-1}$ & $3.5676 \times 10^{-1}$ & 5.3648 & 2.4267 & 2.7655 & 5.0952 & 3.0112 & 5.6340 \\
\hline 9.1 & $3.4435 \times 10^{-1}$ & $4.3626 \times 10^{-1}$ & 9.3077 & 3.1341 & 4.6923 & 8.9633 & 5.0187 & 9.6516 \\
\hline 9.2 & $4.8764 \times 10^{-1}$ & $5.8409 \times 10^{-1}$ & $1.9623 \times 10^{1}$ & 4.4867 & 9.7724 & 1.9135 & $1.0248 \times 10^{1}$ & $2.0110 \times 10^{1}$ \\
\hline 9.3 & $8.6397 \times 10^{-1}$ & $9.6479 \times 10^{-1}$ & $6.4060 \times 10^{1}$ & 8.0352 & $3.1799 \times 10^{1}$ & $6.3196 \times 10^{1}$ & $3.2656 \times 10^{1}$ & $6.4924 \times 10^{1}$ \\
\hline 9.4 & 4.2938 & 4.3989 & $1.6286 \times 10^{3}$ & $4.0363 \times 10^{1}$ & $8.1236 \times 10^{2}$ & $1.6243 \times 10^{3}$ & $8.1665 \times 10^{2}$ & $1.6329 \times 10^{3}$ \\
\hline 9.5 & -1.4007 & -1.2915 & $1.7656 \times 10^{2}$ & $-1.3306 \times 10^{1}$ & $8.9178 \times 10^{1}$ & $1.7796 \times 10^{2}$ & $8.7781 \times 10^{1}$ & $1.7516 \times 10^{2}$ \\
\hline 9.6 & $-5.9755 \times 10^{-1}$ & $-4.8422 \times 10^{-1}$ & $3.2402 \times 10^{1}$ & -5.7362 & $1.6695 \times 10^{1}$ & $3.3000 \times 10^{1}$ & $1.6107 \times 10^{1}$ & $3.1804 \times 10^{1}$ \\
\hline 9.7 & $-3.7936 \times 10^{-1}$ & $-2.6198 \times 10^{-1}$ & $1.3031 \times 10^{1}$ & -3.6796 & 6.9011 & $1.3410 \times 10^{1}$ & 6.5361 & $1.2651 \times 10^{1}$ \\
\hline 9.8 & $-2.7845 \times 10^{-1}$ & $-1.5702 \times 10^{-1}$ & 6.9277 & -2.7286 & 3.8013 & 7.2062 & 3.5423 & 6.6491 \\
\hline 9.9 & $-2.2078 \times 10^{-1}$ & $-9.5297 \times 10^{-1}$ & 4.2477 & -2.1855 & 2.4361 & 4.4685 & 2.2398 & 4.0268 \\
\hline
\end{tabular}

